University of Tennessee Health Science Center

UTHSC Digital Commons

\title{
Arid1a Haploinsufficiency Initiates Neural Crest Transformation in a Mouse Model of Mycn-driven Neuroblastoma
}

Kirby A. Wallace

University of Tennessee Health Science Center

Follow this and additional works at: https://dc.uthsc.edu/dissertations

Part of the Developmental Biology Commons, and the Medicine and Health Sciences Commons

\section{Recommended Citation}

Wallace, Kirby A. (https://orcid.org/0000-0002-2293-3894), "Arid1a Haploinsufficiency Initiates Neural Crest Transformation in a Mouse Model of Mycn-driven Neuroblastoma" (2020). Theses and Dissertations (ETD). Paper 510. http://dx.doi.org/10.21007/etd.cghs.2020.0495. 


\title{
Arid1a Haploinsufficiency Initiates Neural Crest Transformation in a Mouse Model of Mycn-driven Neuroblastoma
}

\author{
Abstract \\ genetically modified primary cells into mice to determine if Arid1a loss collaborates with Mycn \\ pathological features and gene expression profiles of resulting tumors, and evaluated the Arid1a- \\ loss caused a gene expression and phenotypic shift to an immature cell identity. Furthermore, \\ in cell identity may be connected to NBL initiation. \\ Document Type \\ Dissertation \\ Degree Name \\ Doctor of Philosophy (PhD) \\ Program \\ Biomedical Sciences \\ Research Advisor \\ Kevin W. Freeman, Ph.D. \\ Keywords \\ 1p36, development, oncogene, pediatric cancer, stem cells, tumor suppressor \\ Subject Categories \\ Developmental Biology | Medicine and Health Sciences
}

Mouse models of cancer are critical for developing therapeutic treatments for pediatric patients. Recent sequencing studies of neuroblastoma (NBL) patient tumors have uncovered precise deletions in the chromatin remodeler and tumor suppressor gene (TSG) ARID1A. Additional causal studies supported ARID1A's candidacy as a putative 1p36 TSG in MYCN-driven NBL. This study aimed to causally test Arid1a loss during Mycn-driven NBL initiation through the development of a mouse model of high risk NBL.In this study, we modified a Mycn-driven mouse model of NBL to incorporate Cre mediated deletion of floxed Arid1a. Briefly, in freshly isolated embryonic day 9.5 primary trunk neural crest cells (NCCs), Arid1a was heterozygously and homozygously deleted in combination with Mycn overexpression. We injected these overexpression during NCC transformation. We evaluated tumor growth kinetics, characterized the dependent differentiation traits of tumors and primary NCCs in vivo and in vitro. We found that Arid1a heterozygous loss of Arid1a during transformation of primary murine NCCs resulted in tumors that pathologically and molecularly model human high-risk, MYCN amplified NBL. Gene set enrichment analysis demonstrated that gene expression profiles of Arid1a heterozygous tumor samples significantly correlated with previously established mesenchymal gene signatures found in high-risk NBL patients. Our model causally tested the clinical observation that $70 \%$ of high-risk MYCN amplified NBL patient tumors include $1 \mathrm{p} 36 \mathrm{LOH}$. Our results support the hypothesis that ARID1A is a $1 \mathrm{p} 36$ tumor suppressor candidate that collaborates with MYCN to transform NCCs into high-risk NBL. Last, our model suggests that a shift 


\section{Arid1a Haploinsufficiency Initiates Neural Crest Transformation in a Mouse Model of Mycn-driven Neuroblastoma}

Author:

Kirby A. Wallace
Advisor:

Kevin W. Freeman, Ph.D.

A Dissertation Presented for The Graduate Studies Council of

The University of Tennessee Health Science Center

in Partial Fulfillment of the Requirements for the

Doctor of Philosophy degree from

The University of Tennessee

in

Biomedical Sciences/Cancer and Developmental Biology

College of Graduate Health Sciences

May 2020 
Copyright (C) 2020 by Kirby A. Wallace.

All rights reserved. 


\section{ACKNOWLEDGEMENTS}

It is a privilege and a pleasure to have worked with my doctoral training research advisor Dr. Kevin Freeman. His guidance expanded my interest in science and my ability to pursue scientific knowledge philosophically and experimentally. The skills and approaches to problem solving that I gained through working with Dr. Freeman will benefit me throughout my entire career. I wish him all the best in his future endeavors!

I would like to thank my committee members for their attention to and support of my project throughout graduate school. Each committee member has contributed to my scientific training and for that I am extremely grateful. I would especially like to thank Dr. Andy Davidoff for his significant role in resourcing and supporting my doctoral research project.

My previous and current lab members have taught me many scientific and life lessons during my doctoral program. I would like to thank each person for their time spent helping me move this work forward. Thank you to Rachelle Olsen and Joel Otero for their early help getting this project off the ground and thank you to the new lab members for inspiring the continuity of this work.

Graduate school would not have been the same without Dr. Jesus García-López, a post-doctoral scientist I had the privilege of working with and learning from during my time in the Freeman lab. His perspective during the early days of my project helped me see the beauty of science, and his advice throughout graduate school helped me navigate tough hurdles.

Finally, I would like to sincerely thank a new Freeman lab member Chelsea DeVaux for the contributions she made to this project. I am very grateful for her willingness to learn and to jump in to move experiments forward, and I wish her all the best as she continues her journey in science.

I am grateful for my time working in the Davidoff lab, and would like to thank each member of the team for their inclusiveness and support of my project. I would especially like to thank Dr. Dongli $\mathrm{Hu}$ for his help and advice during critical experiments and Mark Brimble for his support of this project and scientific camaraderie.

This research project would not have been possible without the support of the many thoughtful scientists I was fortunate enough to work with. The Microscopy Core, Flow Cytometry Core, and Veterinary Pathology Core at St Jude Children's Research Hospital made meaningful contributions. I am grateful for the contributions made by Krista Millican during critical experiments, and appreciate the opportunity to work with her. Notably, Dr. Heather Tillman was my longest standing collaborator and she contributed significantly to the completion of this project. I learned many scientific and life lessons from her throughout graduate school and it was a privilege to work with her. 
The bioinformatics portion of this project would not have been possible without collaborative help from Dr. Neil Hayes and Jeremiah Holt. Jeremiah's work and the support from Dr. Neil Hayes positively impacted the outcome of this project. Jeremiah's willingness to collaborate across laboratory projects helped me considerably while working with him and I wish him well as he continues his $\mathrm{MD} / \mathrm{PhD}$ training.

I would also like to thank those in the Department of Oncology and the Department of Surgery at St Jude for their support. I would especially like to thank Dr. Mark Hatley and Dr. Jun Yang for their interest in my project and for their suggestions. I would also like to thank them and their lab members for their generosity in sharing reagents, equipment and time. It was a pleasure to learn from and work with all of them.

I would like to sincerely thank the following colleagues for their thoughtful dissertation review and comments: Dr. Jesus García-López, Ph.D.; Rachael Wood; Dr. Carolyn Jablonowski, Ph.D.; and Dr. Lisa Miller, Ph.D.; I am grateful for the time invested in reading my work and giving meaningful feedback.

I would like to thank my family members for their support of my academic goals, especially my mother, Jill Wallace, my father, Truman Wallace and my sister Kaitlin Wallace for their encouragement and unwavering support of my graduate school endeavors no matter how challenging.

I would like to thank Dr. Danny Lewis for launching me on the path of cancer research. I would not have had the opportunities to pursue a career in science and my eventual Ph.D. without him. His mentorship throughout my career so far has helped me in ways that no graphs can measure.

I would also like to thank the friends I've made in graduate school for their kind words and companionship that endured from start to finish of this journey. I would like to thank Lee Pribyl for his encouragement to participate in student government. It was a pleasure to serve on many UTHSC councils with him. I would especially like to thank Rachael Wood for her friendship and camaraderie. Her steadfast attitude toward scientific challenges demonstrates the strength of her character that is also exemplified by her kindness and loyalty as a friend.

Last, I cannot begin to express my thanks to my husband, Patrick Woods. He listened to endless amounts of scientific conjecture and speculation about experimental decisions with unlimited patience and kindness. His excitement about my professional accomplishments helped me experience graduate school as a joy and a privilege and his support was pivotal in helping me complete my degree.

This work would not have been possible without grant funding from the Department of Defense (DOD W81XWH1810477 and DOD W81XWH1410090). 


\begin{abstract}
Mouse models of cancer are critical for developing therapeutic treatments for pediatric patients. Recent sequencing studies of neuroblastoma (NBL) patient tumors have uncovered precise deletions in the chromatin remodeler and tumor suppressor gene (TSG) ARID1A. Additional causal studies supported ARIDIA's candidacy as a putative 1 p36 TSG in $M Y C N$-driven NBL. This study aimed to causally test Aridla loss during Mycn-driven NBL initiation through the development of a mouse model of high risk NBL.
\end{abstract}

In this study, we modified a Mycn-driven mouse model of NBL to incorporate Cre mediated deletion of floxed Aridla. Briefly, in freshly isolated embryonic day 9.5 primary trunk neural crest cells (NCCs), Aridla was heterozygously and homozygously deleted in combination with Mycn overexpression. We injected these genetically modified primary cells into mice to determine if Aridla loss collaborates with Mycn overexpression during NCC transformation. We evaluated tumor growth kinetics, characterized the pathological features and gene expression profiles of resulting tumors, and evaluated the Aridla-dependent differentiation traits of tumors and primary NCCs in vivo and in vitro.

We found that Aridla loss caused a gene expression and phenotypic shift to an immature cell identity. Furthermore, heterozygous loss of Aridla during transformation of primary murine NCCs resulted in tumors that pathologically and molecularly model human high-risk, $M Y C N$ amplified NBL. Gene set enrichment analysis demonstrated that gene expression profiles of Aridla heterozygous tumor samples significantly correlated with previously established mesenchymal gene signatures found in high-risk NBL patients.

Our model causally tested the clinical observation that 70\% of high-risk $M Y C N$ amplified NBL patient tumors include 1p36 LOH. Our results support the hypothesis that $A R I D 1 A$ is a $1 \mathrm{p} 36$ tumor suppressor candidate that collaborates with $M Y C N$ to transform NCCs into high-risk NBL. Last, our model suggests that a shift in cell identity may be connected to NBL initiation. 


\section{TABLE OF CONTENTS}

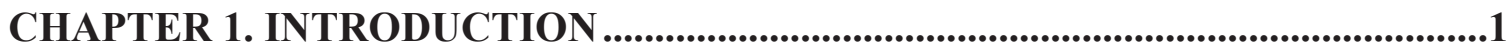

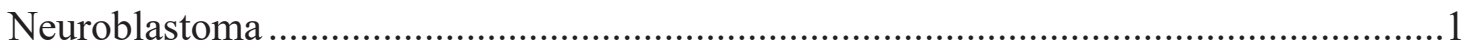

Current neuroblastoma therapy options ......................................................... 1

Chromosomal lesions that define high risk neuroblastoma ...................................2

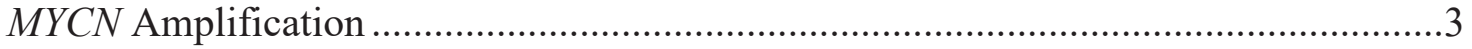

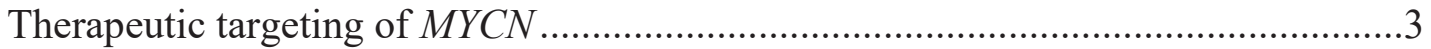

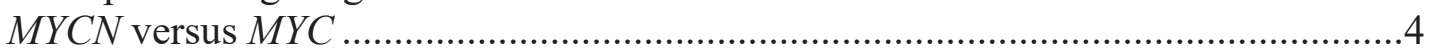

The frontiers of $M Y C N$ research ................................................................ 4

$M Y C N$ amplification and its correlation with $1 \mathrm{p} 36$ loss of heterozygosity .................5

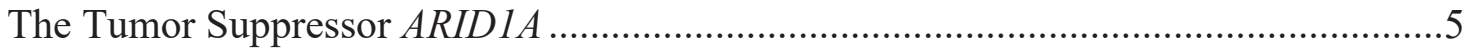

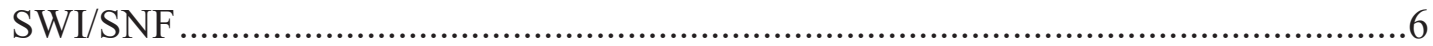

Types of ARID1A mutations .............................................................................

Therapeutic targeting of $A R I D 1 A$ mutant tumors ................................................... 7

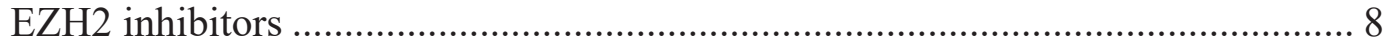

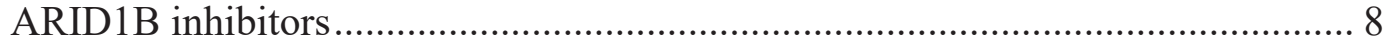

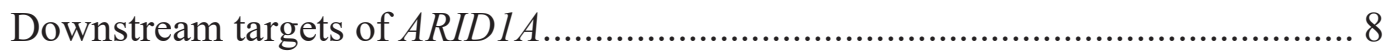

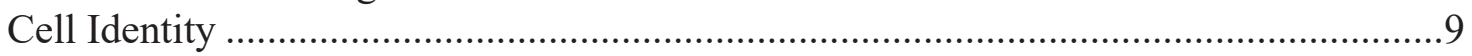

Neural crest cells ......................................................................................... 10

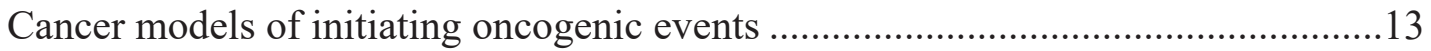

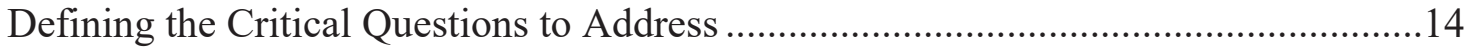

What is the oncogenic action of MYCN? ............................................................ 14

Does loss of Aridla result in a synergistic relationship with $M Y C N ? \ldots \ldots \ldots \ldots \ldots \ldots \ldots . . . .15$

CHAPTER 2. METHODOLOGY .........................................................................17

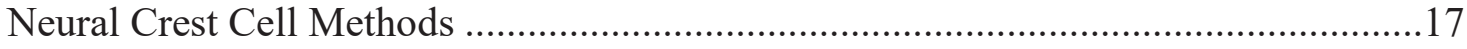

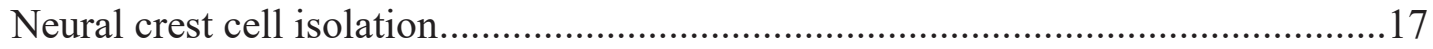

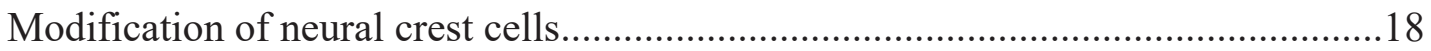

Tumor measurement cutoffs and statistics.......................................................... 18

Tumor studies in SCID mice............................................................................ 18

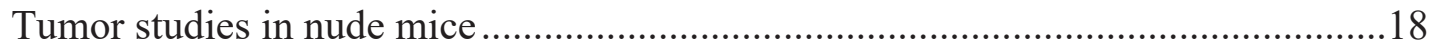

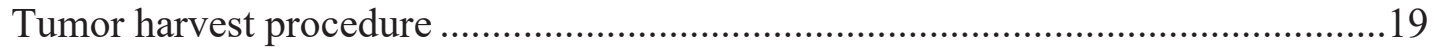

Derivation of cell lines from fresh tumor sample ..................................................19

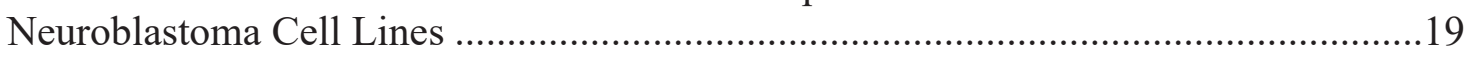

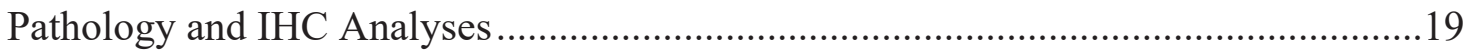

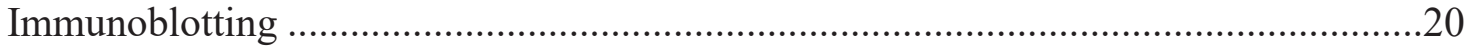

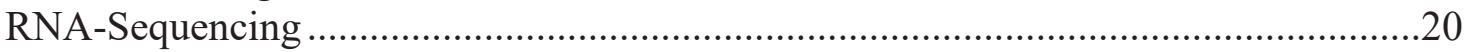

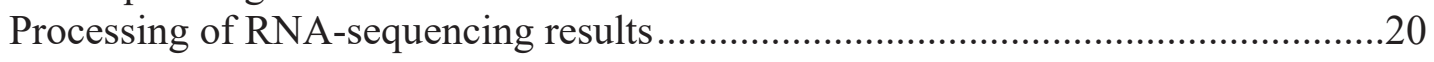

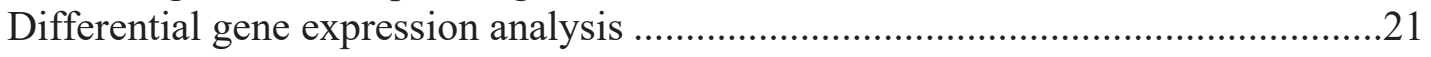

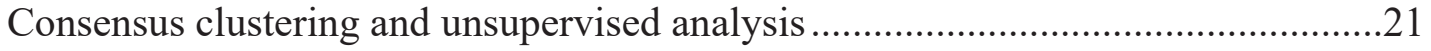

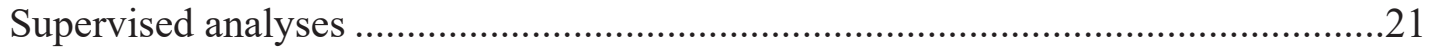




\section{CHAPTER 3. ARID1 $A$ IS A CRITICAL TUMOR SUPPRESSOR GENE IN $1 P 36$ DELETED, MYCN AMPLIFIED NEUROBLASTOMA}

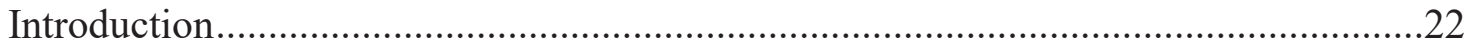

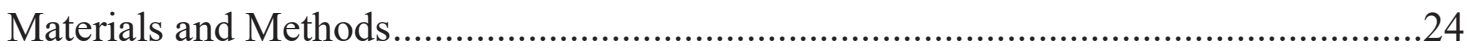

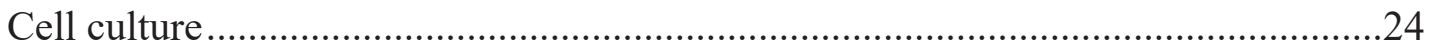

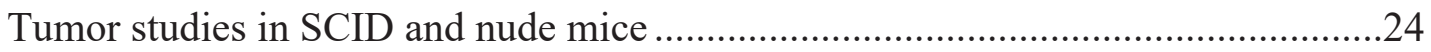

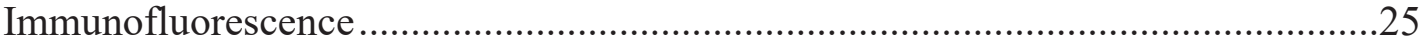

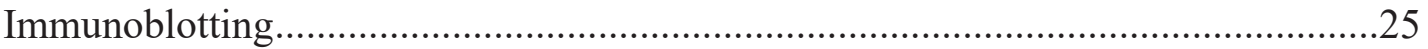

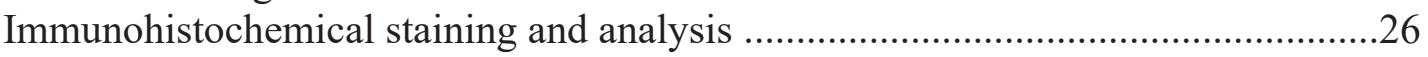

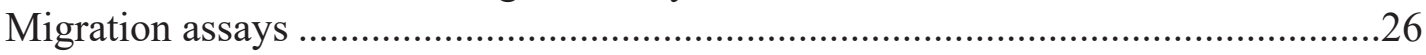

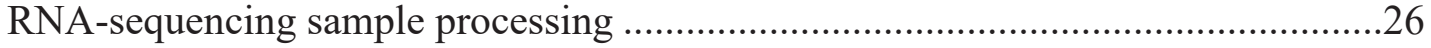

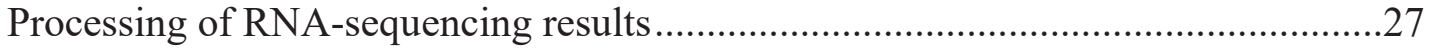

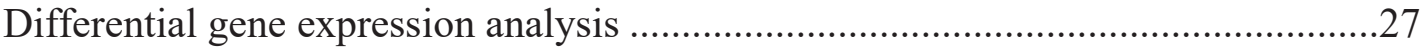

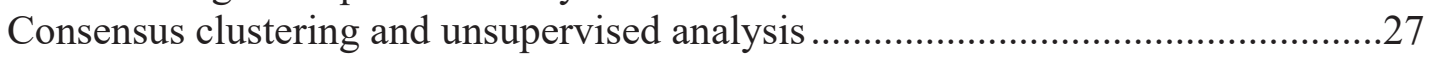

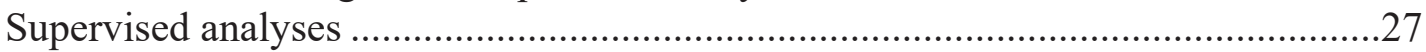

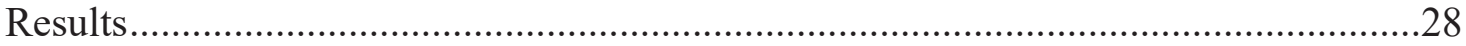

Haploinsufficient Aridla is a driver mutation that collaborates with $M Y C N$ in

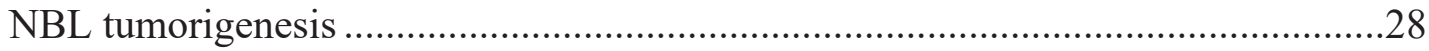

Gene expression and pathological and molecular characterization of in vivo

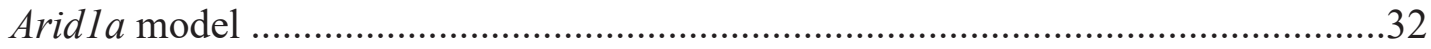

Aridla loss causes a differentiation block in vivo and in vitro ...............................36 Aridla loss up-regulates genes associated with a mesenchymal cell identity ...........38 Aridla heterozygosity correlates with an immature, mesenchymal cell state ...........45 Arid1a heterozygous tumors gene signature recapitulates human NBL

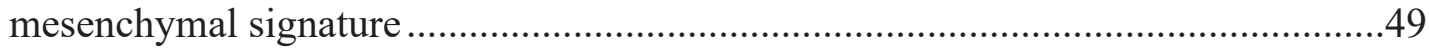

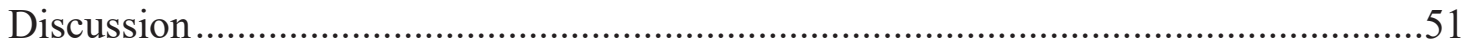

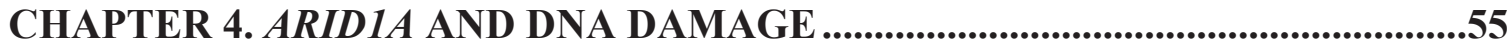

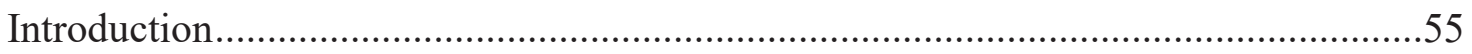

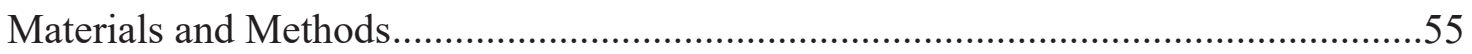

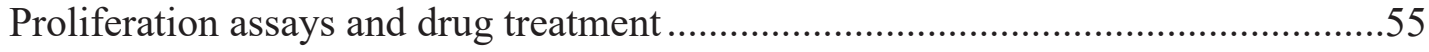

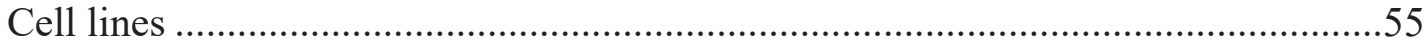

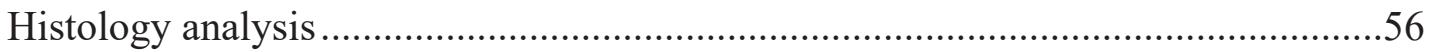

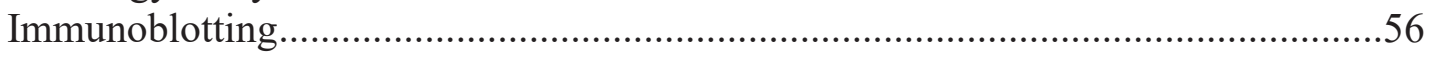

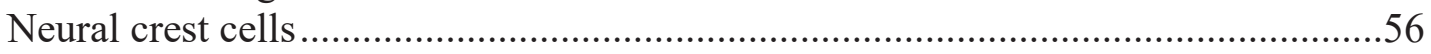

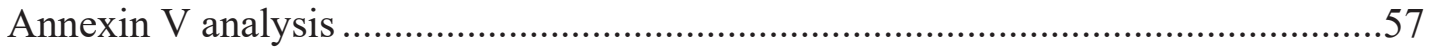

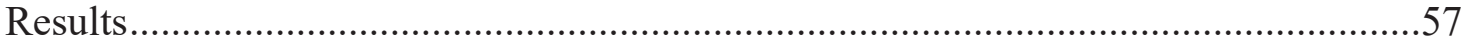

ARID1A protein levels are increased in MNA cell lines .......................................57

Characterization of DNA damage and cell death in end-stage tumors .....................60

Aridla heterozygous and homozygous tumor derived cell lines do not have

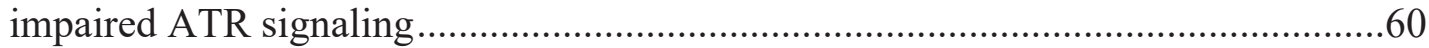

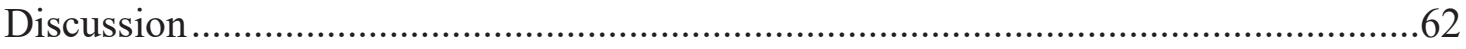

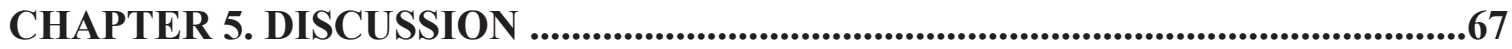

Significance of Aridla Findings in the NBL Community ........................................67 
Connecting causal gene mutations and patient outcomes

Genetic mutations that disrupt NCC identity may contribute to NBL initiation .......67

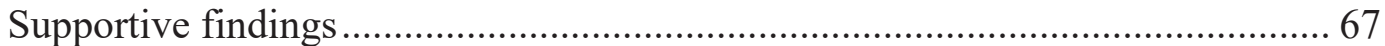

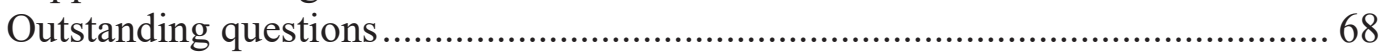

Hunt for the 1p36 Tumor Suppressor Gene .........................................................69

Collaborative ARIDIA and MYCN gene expression programs ............................... 70

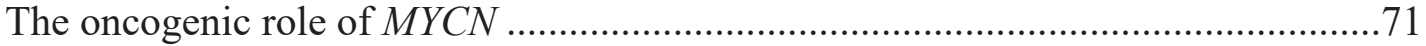

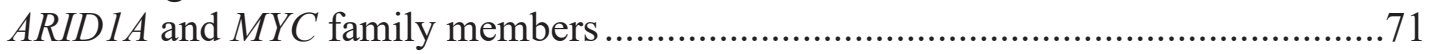

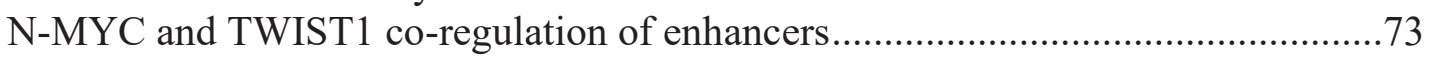

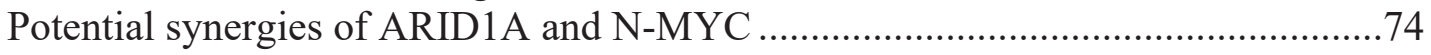

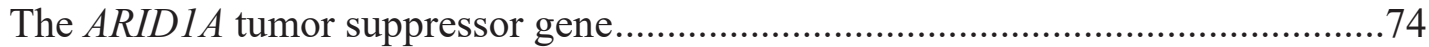

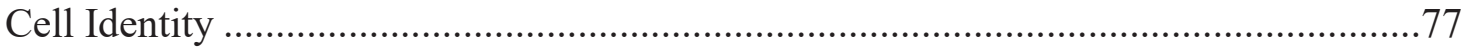

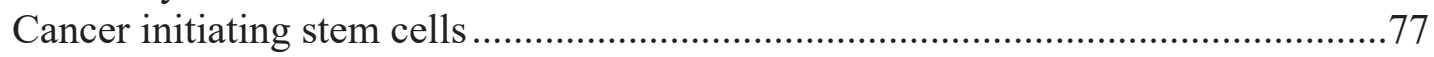

Cell identity master regulators .................................................................... 78

Aridla heterozygous tumors model human mesenchymal NBL ............................81

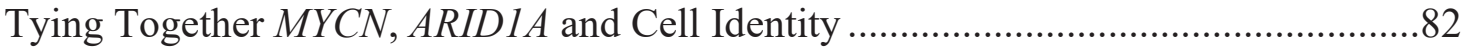

A working model connecting ARIDIA loss, MYCN enhancer invasion and TF

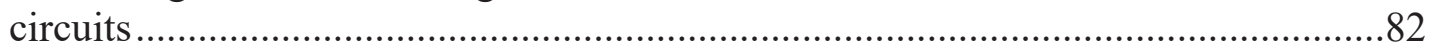

Disease modeling - key features and limitations ................................................ 83

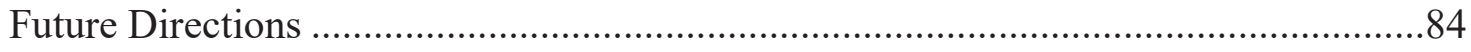

Functional validation of the Aridla-regulated gene signature ...............................85

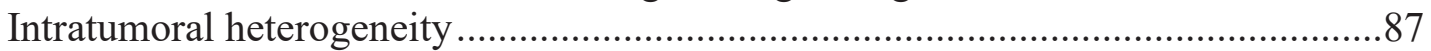

Modeling and testing metastatic disease ........................................................ 90

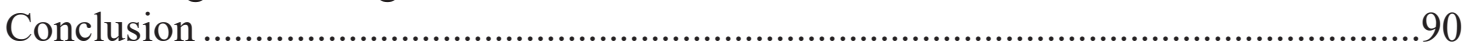

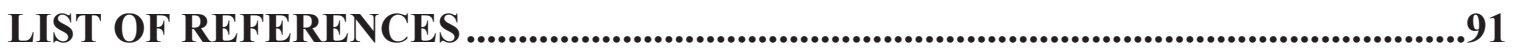

APPENDIX A. ADDITIONAL MATERIALS AND METHODS TECHNICAL

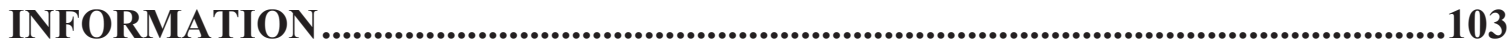

APPENDIX B. SUPPLEMENTAL EXPERIMENTAL FIGURES .........................107

VITA 


\section{LIST OF TABLES}

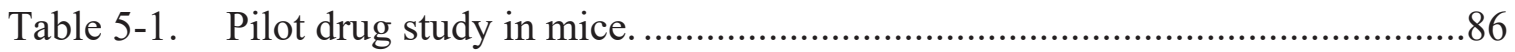

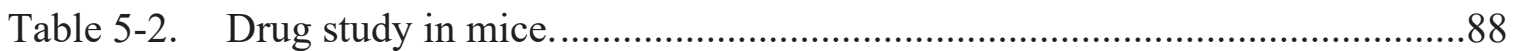

Table 5-3. Validation of xenograft growth in mice................................................ 88

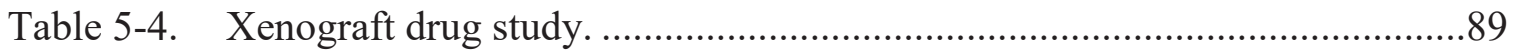

Table A-1. Predicted ARIDIA genetic status in common neuroblastoma cell lines. ....103

Table A-2. Immunohistochemistry primary antibodies, type, concentrations, supplier and technical procedures for visualization. ..............................105

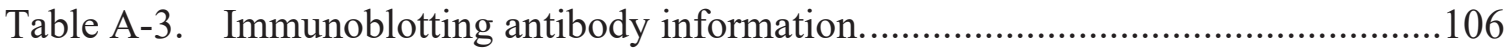




\section{LIST OF FIGURES}

Figure 1-1. Schematic of neural crest origins.

Figure 3-1. Heterozygous Ardila deletion increases transformation kinetics of Mycn overexpression in primary trunk NCCs.

Figure 3-2. Grouped data recapitulates trends in Kaplan-Meier limiting dilution graphs.

Figure 3-3. Heterozygous Aridla synergizes with MYCN-driven NBL

Figure 3-4. Loss of Aridla expression is consistent with Aridla genotype in end point tumors.

Figure 3-5. RNA-sequencing and histological characterization distinguishes HET

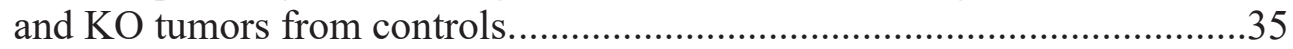

Figure 3-6. Aridla loss blocks differentiation in vivo and in vitro. 39

Figure 3-7. CRISPR-mediated deletion of Aridla impaired neuronal differentiation. ...41

Figure 3-8. Arid1a-dependent up-regulated signaling nodes.

Figure 3-9. Arid1a loss disrupts EMT in vitro and in vivo. . .44

Figure 3-10. Tumor fate mapping reveals differences in HET and KO tumor samples....46

Figure 3-11. Differential gene expression analysis reveals alterations of NCC master regulators in tumor samples.

Figure 3-12. Mouse model comparison to human NBL adrenergic and mesenchymal signatures.

Figure 4-1. N-MYC and ARID1A protein expression are correlated in NBL and NCC samples.

Figure 4-2. Aridla status was not correlated to apoptotic response when Mycn was titrated into primary NCCs.

Figure 4-3. DNA damage and cell death analysis of tumors.

Figure 4-4. Aridla HET and KO tumor derived cell lines do not have impaired ATR signaling in response to etoposide treatment.

Figure 4-5. Aridla HET and KO tumor derived cell lines do not have impaired ATR signaling in response to topotecan treatment. 
Figure 4-6. 1p36 LOH did not alter ATR signaling in topotecan treated NBL cells. .....65

Figure B-1. Comparison of ARID1A and N-MYC protein levels in WT1 and HET primary NCCs.

Figure B-2 Enrichment of BAF155 protein following immunoprecipitation and immunoblotting. 


\section{LIST OF ABBREVIATIONS}

\begin{tabular}{|c|c|}
\hline $\mathrm{CDM}$ & Chemically Defined Media \\
\hline $\mathrm{COG}$ & Children's oncology group \\
\hline GEMM & Genetically engineered mouse model \\
\hline HET & Heterozygous \\
\hline $\mathrm{HOM}$ & Homozygous \\
\hline HR & High risk \\
\hline $\mathrm{LOH}$ & Loss of heterozygosity \\
\hline MKI & Mitotic- karyorrhexis index \\
\hline MNA & MYCN amplification \\
\hline NBL & Neuroblastoma \\
\hline $\mathrm{OE}$ & Overexpression \\
\hline SCID & Severely compromised immunodeficient \\
\hline SL & Synthetically lethal \\
\hline SMI & Small molecule inhibitor \\
\hline SNS & Sympathetic nervous system \\
\hline SWI/SNF & Switch/sucrose non-fermenting \\
\hline $\mathrm{TF}$ & Transcription factor \\
\hline TSG & Tumor suppressor gene \\
\hline WES & Whole exome sequencing \\
\hline
\end{tabular}




\section{CHAPTER 1. INTRODUCTION}

\section{Neuroblastoma}

NBL is the most common extracranial pediatric solid tumor, and accounts for 15 percent of pediatric cancer deaths each year. NBL tumors are inherently heterogeneous, which results in a disease with poor prognosis and outcomes. The children's oncology group (COG) stratifies patients into one of three risk groups at diagnosis: low, intermediate, or high (Coughlan et al., 2017; Davidoff, 2012). Forty percent of patients diagnosed are HR with metastatic spread with primary disease arising in the adrenal glands (Coughlan et al., 2017). HR NBL is classified based on Shimada histology, mitotic-karyorrhexis index (MKI), Schwannian stromal content, age at diagnosis, and MYCN amplification (MNA) (Davidoff, 2012; Jiang et al., 2013; Peuchmaur et al., 2003; Shimada et al., 1999). Age at diagnosis has been shown to correlate with patient outcomes. Patients diagnosed at $<18$ months of age are most likely to be low or intermediate risk, while patients $>18$ months are more likely to be diagnosed with HR disease (Davidoff, 2012; J. R. Park et al., 2013). Furthermore, most HR patients are between 18 months and 12 years old (Davidoff, 2012). Current patient stratification approaches highlight the uneven distribution of patient outcomes across risk groups. Low and intermediate-risk patients have positive prognosis and a 95\% chance of survival; however, HR patients have a much worse prognosis of event free survival and a 30-50\% chance of 5-year survival (Coughlan et al., 2017; Davidoff, 2012; Pinto et al., 2015; Tolbert \& Matthay, 2018).

\section{Current neuroblastoma therapy options}

NBL treatment regimens are dependent on the stage of tumor at diagnosis and rely on patient groups defined by either the COG clinical trial protocol, or the International Neuroblastoma Risk Group (INRG) system (Coughlan et al., 2017; Davidoff, 2012). According to COG protocol, risk stratification determines treatment protocol, with the number of treatments increasing as disease severity increases. For example, low risk patients often receive no chemotherapy treatment or are treated with a chemotherapy regimen consisting of 1 or more agents paired with surgery(Coughlan et al., 2017; Davidoff, 2012). Outcomes measure near 100\% for 5-year survival rates (Coughlan et al., 2017). Intermediate NBL patients are treated with four or more chemotherapeutic agents paired with surgery, and 5-year survival rates are stable at $93 \%$ (Coughlan et al., 2017; Davidoff, 2012; Nakagawara et al., 2018). In contrast to these two groups, HR patients receive an arsenal of treatments. Patients receive cycles of chemotherapy that include eight or more types of chemotherapeutic agents (Coughlan et al., 2017). Furthermore, surgery is required, and additional therapies may be paired with these two treatments. Additional therapeutic options often used to treat HR patients include myeloablative radiation followed by stem cell transplant, along with GD2 immunotherapy, and retinoic acid differentiation therapy(Coughlan et al., 2017; Davidoff, 2012). Five-year survival rates for these patients recently measured at $46 \%$ 
(Coughlan et al., 2017; García-López et al., 2020). In summary, risk stratification correlates with outcomes. Fewer treatments are required to treat low and intermediaterisk disease - fortunately producing great outcomes; however, therapeutic regimens for HR patients are intensive and outcomes are poor (Coughlan et al., 2017; Davidoff, 2012; J. R. Park et al., 2013; Tolbert \& Matthay, 2018). Consequently, the development of precision medicine that incorporates newly identified biomarkers and therapeutic agents remains integral to improving outcomes and patient lives (D. T. W. Jones et al., 2019; J. R. Park et al., 2013; Pugh et al., 2013).

We propose that improving outcomes will be addressed through identifying the oncogenic drivers of this disease. We hypothesize that furthering the field's knowledge of the initiating tumorigenic events of HR disease will provide new insights into the disease origin. Our aim is to expose underlying weaknesses of HR NBL that can be therapeutically exploited. The following literature review highlights three key areas of research: chromosomal abnormalities that underlie HR NBL, conclusions that can be drawn from other fields of study describing our genes of interest, and the unresolved questions we aim to answer.

\section{Chromosomal lesions that define high risk neuroblastoma}

Intratumoral heterogeneity that correlates to patient outcomes has been connected to the genetic events that underlie NBL (Garrett M. Brodeur, 2003). One approach to improving our understanding of how this disease will progress is identification and evaluation of molecular and cellular consequences of the genetic lesions harbored in NBL tumors (Garrett M. Brodeur, 2003; Garrett M. Brodeur et al., 2014). Many chromosomal gains and losses are evident in NBL, notably gain of 17q, MYCN amplification, and loss of material at 11q, 14q, and 1p (Attiyeh et al., 2005; Garrett M. Brodeur, 2003; Davidoff, 2012; C. T. Fong et al., 1989; Jiang et al., 2013). Despite the frequency of genetic lesions, the only chromosomal event currently used to clincially stratify patients is $M Y C N$ amplification (Tolbert \& Matthay, 2018). However, the genetic perturbations found in NBL demonstrate patterns of overlap, suggesting either synergy, or mutual exclusivity; identification of cooperating mutations may provide new therapeutic opportunities (Garrett M. Brodeur et al., 2014). One illustration of this concept is loss of heterozygosity at 1p36.1-1p36.3 has perplexed the NBL community for over 30 years (C. T. Fong et al., 1989) Interestingly, this region is an example that demonstrates both overlapping and mutually exclusive genetic events. This is highlighted by the work published twenty four years ago. Caron, et al identified two regions of deletion in 1p36, a distal and proximal region (Caron et al., 1995). The distal region of deletion is mutually exclusive of $M Y C N$, and contains the smallest region of common deletion (Caron et al., 1995; García-López et al., 2020; White et al., 2005). The larger proximal region of deletion coincides with MYCN amplification (MNA) (Caron et al., 1995; García-López et al., 2020). The combination of proximal deletion of $1 \mathrm{p} 36$ and $M Y C N$ amplification are correlated in 15$20 \%$ of all NBL cases. Tumors with 1 p3 6 loss of heterozygosity (LOH) and MYCN amplification are HR, frequently metastatic, frequently relapse post-therapy and result in 
poor patient outcomes with survival rates of approximately 30 percent (Attiyeh et al., 2005; Caron et al., 1995; Jiang et al., 2013).

\section{MYCN Amplification}

MYCN is one of three members of the MYC family of bHLH-LZ transcription factors that promote the expression of their gene targets by binding to promoters and enhancers (Rickman, Schulte, \& Eilers, 2018; Ruiz-Pérez, Henley, \& ArsenianHenriksson, 2017). During development, N-myc is expressed in a restricted number of tissues and is responsible for the rapid proliferation of the developing central nervous system (CNS) (Rickman et al., 2018). In cancer, the MYC gene family is frequently deregulated, often via gene amplification (Lin et al., 2012; Rickman et al., 2018; Van Dang et al., 2016). MYCN is an oncogene that is overexpressed in five nervous system cancers, and in six non-neuronal tumors (Rickman et al., 2018). MYCN amplification (MNA) was discovered in year 1983 by Schwab and colleagues and was defined as an oncogenic driver of NBL (Schwab, 1983, Nature). MNA often results in 50 to 400 gene copies per cell and a corresponding increase in N-Myc protein expression (Maris \& Matthay, 1999). MNA is a predominant driving mutation in NBL and is used as a biomarker of tumor aggressivity at diagnosis that designates HR disease. In NBL, MYCN is not clonally acquired, and has been shown to initiate NBL across multiple species in experimental models, implicating it as a driving oncogenic lesion in NBL (García-López et al., 2020; Olsen et al., 2017; Rickman et al., 2018; Weiss, Aldape, Mohapatra, Feuerstein, \& Michael Bishop, 1997).

\section{Therapeutic targeting of $M Y C N$}

In pre-clinical models of NBL performed by multiple research groups, $M Y C N$ expression during varying stages of SNS development initiated NBL across mouse, zebrafish, and primary cell models (García-López et al., 2020; Olsen et al., 2017; Rickman et al., 2018; Tsubota \& Kadomatsu, 2018; Weiss et al., 1997; Zhu et al., 2012). Despite knowing for decades that $M Y C N$ amplification is fundamental to NBL oncogenesis, development of inhibitors for clinical use has lagged due to several physical limitations of precision medicine. First, transcription factors (TFs) that reside in the nucleus are difficult to effectively target, which limits drug delivery options (Garrett M. Brodeur et al., 2014). Second, unbound $M Y C N$ is largely unstructured, which results in a lack of binding pockets (Rickman et al., 2018; Ruiz-Pérez et al., 2017). Third, the structure of $M Y C N$ is determined by its binding partner MAX, and crystal structures of the heterodimer do not indicate obvious targetable sites (Rickman et al., 2018; Ruiz-Pérez et al., 2017). Therefore, there are currently no target-specific treatments for HR MYCN amplified NBL. Taken together, an alternative approach to therapeutic development for $M Y C N$-driven NBL is necessary. 


\section{MYCN versus $M Y C$}

MYC is the founding member of the MYC family. There are three family members that include $M Y C, M Y C N$, and MYCL (C-Myc, N-Myc, and L-Myc proteins, respectively). $M Y C$ is the most frequently mutated gene in this family, and consequently has been studied across the broadest variety of cancers (Dang, 2012; Lin et al., 2012). Review articles often refer to $M Y C$ as a starting point for understanding the characteristics and oncogenic effects of $M Y C N$. In the past, $M Y C$ and $M Y C N$ were considered to behave with similar activity, and differences were attributed to differential temporal or localized expression (Malynn et al., 2000). However, as studies expand, differences between these two genes and their differential regulation of genes is evident, especially in the context of cancer. To highlight this point, differences in MYC activity and binding across a variety of cancer types has been linked to "C-Myc target signatures" (Lin et al., 2012). However, even the C-Myc-dependent targets display little overlap across different cancers and are considered cancer type specific (Lin et al., 2012). Independent studies of $M Y C$ and $M Y C N$ amplified cancers suggest a similar method of oncogenic action that is exerted on cancer-specific gene targets (Lin et al., 2012; Zeid et al., 2018).

\section{The frontiers of $M Y C N$ research}

An emerging concept regarding MYC family proteins is "enhancer invasion." Both C-Myc and N-Myc have been shown to preferentially bind high-affinity promoters, but in cases of oncogenic overexpression of these proteins, they begin binding, or invading, low-affinity promoters (Lin et al., 2012; Rickman et al., 2018; Van Dang et al., 2016; Zeid et al., 2018). One interpretation of enhancer invasion in cancers with MYC or $M Y C N$ amplification is that the epigenetic landscape predetermines the genetic profile that is then modulated by MYC/MYCN (Lin et al., 2012; Rickman et al., 2018). Briefly, $M Y C$ and $M Y C N$ have similar promoter binding signatures, and recent studies have suggested that the differences seen in $M Y C$ or $M Y C N$ gene expression profiles are due to their binding of open enhancer regions when overexpressed, as in amplified cases (Zeid et al., 2018). The differential gene expression patterns seem to be tissue specific and determined by two factors: 1 . the epigenetic landscape that controls open enhancer regions, and 2. other cooperating enhancer binding proteins (Lin et al., 2012; Zeid et al., 2018). Therefore, the concept of enhancer-driven transcription is one rationale that may explain the cancer-type-dependent roles of MYCN (Zeid et al., 2018). In summary, the most promising approaches to targeting $M Y C N$ overexpressed tumor types are the identification of MYCN regulated downstream events that can be therapeutically exploited. Establishing the enhancer regulating proteins that synergize with $M Y C N$ may uncover therapeutic vulnerabilities in key pathways. 


\section{$M Y C N$ amplification and its correlation with 1 p36 loss of heterozygosity}

Deletions on 1p were first discovered in 1977 by Brodeur, et al., and are correlated with MYCN amplification (G M Brodeur, Sekhon, \& Goldstein, 1977; C. T. Fong et al., 1989). The high frequency of 1 p36 LOH suggests that deletion of this region is a driving event in NBL and that $1 \mathrm{p} 36$ harbors one or more tumor suppressor genes (TSGs) (Garrett M. Brodeur, 2003; C. T. Fong et al., 1989; Versteeg et al., 1995). 1p36 $\mathrm{LOH}$ is a hemizygous deletion, without reduplication, found on one of the two sister chromosomal alleles, and gene mapping has identified that approximately 500 genes may be lost in this region (C. T. Fong et al., 1989). Due to the vast number of TSG candidates lost, the second 1p36 allele has been thoroughly studied for additional inactivating point mutations that may correspond to crucial TSG candidates. However, identifying the important gene or genes in this region has been difficult because inactivating point mutations were not found on the second allele until recently. In 2013, using whole exome and whole genome sequencing of 74 distinct patient tumors, Sausen, et al. identified point mutations that resulted in biallelic ARIDIA deletions in 3 cases, and independent ARIDIA point mutations in an additional 3 cases (Sausen et al., 2013). Furthermore, in support of the original findings of Caron and colleagues, when sequences from patient samples in the St Jude TARGET dataset were separated by MYCN status, Aridla was included in the region of deletion at 1p36 only when $M Y C N$ was amplified (García-López et al., 2020). However, when $M Y C N$ was wild-type, Aridla was not always deleted (García-López et al., 2020). Taken together these results suggested that $A R I D 1 A$ inactivation is a loss of function oncogenic event in NBL and positioned ARIDIA as a TSG candidate for MNA NBL.

\section{The Tumor Suppressor $A R I D 1 A$}

Although pediatric cancers have a lower mutational burden than adult cancers, recent large pediatric cancer analyses have identified epigenetic aberrations as the top contributors to pediatric diseases (Gröbner et al., 2018; Lawlor \& Thiele, 2012; Ma et al., 2018). Epigenetics is commonly defined as the regulation of "DNA templated processes" through chromatin modification (Dawson \& Kouzarides, 2012). ARIDIA (AT-Rich interacting domain containing protein $1 \mathrm{~A}$ ) is a TSG that is frequently mutated across 25 adult and pediatric cancer types including NBL (R. C. Wu, Wang, \& Shih, 2014). $A R I D 1 A$ is essential for the ability of the SWI/SNF (Switch/Sucrose Non-Fermenting) chromatin remodeling complex to bind DNA and is the most mutated member of this $\sim 15$ subunit complex (S. Jones et al., 2012; Kadoch et al., 2013; Wiegand et al., 2010; R. C. Wu et al., 2014). ARIDIA has causally been identified as a context dependent TSG (Guan, Wang, \& Shih, 2011; Sun, Wang, et al., 2017; S. C. Wang et al., 2019; R. C. Wu et al., 2014). Aridla serves multiple roles in cellular modulation as a known epigenetic regulator of enhancers (Alver et al., 2017; Mathur et al., 2017; Raab, Resnick, \& Magnuson, 2015; Son \& Crabtree, 2014), cell signaling through P13K and dna damage pathways (Chandler et al., 2015; Hu et al., 2018; Lord et al., 2016; Shen et al., 2018, 
2015) as well as crucial roles in liver and hematopoietic differentiation (Han et al., 2019; Sun, Chuang, et al., 2017; Sun, Wang, et al., 2017).

\section{SWI/SNF}

Mutated epigenetic mechanisms have been proposed as initiating events in cancers with mostly silent genomes (Flavahan, Gaskell, \& Bernstein, 2017), which applies to most childhood cancers including NBL. SWI/SNF is the most mutated chromatin remodeling complex in cancer (Kadoch et al., 2013; J. N. Wu \& Roberts, 2013). One role of chromatin remodelers is to partner with transcription factor genes to define lineages and restrict chromatin accessibility as developmental processes progress (Flavahan et al., 2017). Precisely timed chromatin restriction is required for proper differentiation, and perturbation of this step is proposed as a "major factor" of tumorigenesis (Flavahan et al., 2017). The SWI/SNF complex binds to promoter and enhancer regions, but preferentially binds to and regulates the opening and closing of chromatin at enhancer regions during development to determine lineage commitment (Alver et al., 2017; Raab et al., 2015).

SWI/SNF was identified in yeast and is known as BAF (Brahma associated factor) in human cells (Son \& Crabtree, 2014). As differentiation occurs during development, the BAF complex adapts by acquiring different sub-unit compositions (Raab et al., 2015; Son \& Crabtree, 2014). Whether these adaptations cause differentiation to proceed or are the result of differentiation is currently unclear. Regardless, $A R I D I A$ is present in the complex in mutual exclusivity of other ARID family members, specifically $A R I D 1 B$ and $A R I D 2$. ARID $1 A$ and $A R I D 1 B$ are components of the $\mathrm{BAF}$ complex, which is the most commonly identified complex across tissue types. ARIDIA and ARID 2 are components of pBAF (polybromo BAF), a complex involved in the differentiation of various tissue types (Raab et al., 2015). One study in HepG2 cells used gene silencing technology to sequentially ablate expression of each Arid protein (Raab et al., 2015). Next, the chromatin binding regions of overlap and exclusive binding sites were identified for each Arid protein. Although there were sites of overlap, each protein was shown to differentially bind chromatin sites (Raab et al., 2015). This finding was interesting given the context that Arid proteins have been shown to bind promiscuously to chromatin (Dallas et al., 2000). Furthermore, each Arid protein regulated a different gene program (Raab et al., 2015). It is currently unclear how BAF binding sites are decided. However, this data suggests that genetic mutations or ablation of one ARID protein may cause the complex to be redirected to a new binding location, resulting in new chromatin characteristics, and possibly cellular deregulation.

To summarize, ARID1A deletions and mutations result in a new complex by allowing either Arid1b or Arid 2 to engage with the BAF or pBAF complex, respectively. One possible outcome of this altered composition is the targeting of the SWI/SNF complex to new chromatin sites, resulting in downstream gene expression differences. Whether ARID1B, or ARID 2 will engage in the complex following ARIDIA mutation is dependent on tissue type and developmental stage. One limitation of current cancer 
studies is that mutated SWI/SNF components are identified, but the resulting complex makeup is not often resolved. As demonstrated in studies by Kadoch, et al., immunoprecipitation of SWI/SNF complex components following identification of a subunit mutation, such as Aridla deletion, will be a necessary step in elucidating how SWI/SNF mutations result in a new complex composition that drives cancer and other diseases (Kadoch et al., 2013).

\section{Types of $A R I D 1 A$ mutations}

$A R I D 1 A$ is frequently mutated in ovarian clear cell carcinoma, gastric cancer, breast cancer, pancreatic cancer, prostate cancer, and hepatocellular carcinoma (Kadoch et al., 2013; J. N. Wu \& Roberts, 2013; R. C. Wu et al., 2014). The majority of mutations have been identified in genome sequencing screens and immunohistochemistry (IHC) analyses (Kadoch et al., 2013; J. N. Wu \& Roberts, 2013). Mutations are often frameshift or nonsense and are predicted to be loss of function (Bitler, Fatkhutdinov, \& Zhang, 2015; Mathur, 2018; J. N. Wu \& Roberts, 2013). Of the genetic mutations identified, both homozygous and heterozygous mutations occur. In a large screen of 3,000 tumor samples, only $7 \%$ demonstrated complete $A R I D 1 A$ protein ablation (J. N. Wu \& Roberts, 2013). Overall, IHC based screens on tumor sections resolved that ARIDIA protein expression is often reduced to a range between 75-45\% loss (J. N. Wu \& Roberts, 2013). This suggests that homozygous deletion of ARIDIA may be necessary in some contexts, but heterozygous loss of function may be sufficient in others. Furthermore, ARIDIA is a potential heterozygous TSG in multiple cancer types, which further supports its candidacy as a 1p36 LOH TSG.

\section{Therapeutic targeting of $A R I D 1 A$ mutant tumors}

Due to the high rates of $A R I D I A$ mutations across cancer types, multiple groups are working to identify druggable targets in these $A R I D 1 A$ mutated cancers (Bitler et al., 2017; Ogiwara et al., 2019; R. C. Wu et al., 2014). Targeting loss of function oncogenic drivers has been a long-term issue in therapeutic development. In the 1990s, gene therapy was developed with the idea of correcting loss of function genetic events. Re-expression of the gene of interest using a viral vector that included an engineered copy of the lost gene was expected to correct these loss of function mutations. However, difficulty in delivery, uptake, off-target effects and long-term expression of the new gene insert has significantly impeded the use of this therapeutic option (Birkeland, Ludwig, Spector, \& Brenner, 2016). An alternative approach used to therapeutically target loss of function drivers is the identification of druggable pathways altered following gene loss, or identification of extracellular membrane proteins overexpressed following loss of the gene of interest. The field of therapeutics is rapidly developing new drug options, notably small molecule inhibitors (SMIs) are considered a powerful option for precision therapy in NBL (Garrett M. Brodeur et al., 2014). Recently, efforts to target ARIDIA mutant cancer using precision medicine focus on the development of synthetic lethal small molecule inhibiters (SL-SMIs) (Gorrini \& Mak, 2019; Helming et al., 2014; Ogiwara et 
al., 2019; J. N. Wu \& Roberts, 2013). The Aridla field has currently used SL-SMIs to target three main groups: PRC2 complex members, namely EHZ2 inhibitors, other SWI/SNF complex members, specifically ARID1B, and new candidates that have recently been identified as downstream of ARIDIA.

\section{EZH2 inhibitors}

PRC2 is a chromatin remodeling complex that compacts chromatin and has been shown to antagonize SWI/SNF (B. G. Wilson et al., 2010). EZH2 is the catalytic subunit of this complex, and deposits methyl marks on histone 3 lysine 27 (H3K27), causing repression of gene transcription (B. G. Wilson et al., 2010). In ovarian clear cell carcinoma (CCC), EZH2 inhibitors were found to notably cause apoptosis in Aridla mutant cells (Bitler, Aird, et al., 2015; Bitler, Fatkhutdinov, et al., 2015). EZH2 inhibitors are currently in clinical trials for B-cell lymphoma, and have shown promising results (Bitler, Fatkhutdinov, et al., 2015). In summary, further pre-clinical testing of EZH2 inhibition will be needed to determine if this is a beneficial option for Aridla mutant cancers and possibly other SWI/SNF mutant cancer types (Bitler, Fatkhutdinov, et al., 2015).

\section{ARID1B inhibitors}

In 2014, Helming et al. identified ARID1B as an "Achilles heel" of ARID1A mutant ovarian cancer cell lines (Helming et al., 2014). Inhibition of ARIDIB using shRNA reduced cell proliferation and colony formation in ARIDIA mutant cell lines OVISE and TOV21G, but not ARIDIA wild-type cell lines ES-2 or 293T (Helming et al., 2014). Additionally, depletion of $A R I D I B$ caused the SWI/SNF complex to dissociate in ARIDIA mutant cells (Helming et al., 2014). Together these data suggested that targeting $A R I D I B$ gene products may be a viable therapeutic option for ARIDIA mutant cancer types. However, as discussed above, ARID1B may not be present in the complex in all tissue types. Therefore, ARID1B inhibition may apply to some, but not all ARIDIA mutant cancers. Identification of the SWI/SNF composition in each ARIDIA mutated cancer, and empirical testing of the inhibitors of interest will be necessary for appropriate development of this therapeutic approach.

\section{Downstream targets of $A R I D 1 A$}

Multiple groups have identified potential roles for ARIDIA in DNA damage signaling. Small molecule inhibitors targeting ATR and PARP were tested in the ARIDIA knockout colorectal cells HCT119 by two groups (Lord et al., 2016; Shen et al., 2015). Both compounds were shown to selectively target ARIDIA mutant cells. However, in an additional study, the ovarian cancer cell line MCF10A was depleted of ARIDIA using esiRNA (endonuclease-prepared small interfering RNA), and the cells were screened for chemical-genetic interactions (Hu et al., 2018). Paired with the Cancer Therapeutics Response Portal (CTRP), 496 cells were found to be resistant to etoposide (Hu et al., 2018). Furthermore, 10 patients in clinical trials with rucaparib, a PARP inhibitor were analyzed for progression free survival (Hu et al., 2018). The patients demonstrated 
significantly lower progression free survival than the other cohort patients, suggesting that the PARP inhibitors do not improve patient outcomes in endometroid or high-grade serous ovarian cancer (Hu et al., 2018). Furthermore, prediction and testing of possibly synergistic DNA damaging agents may improve pre-clinical results (Hu et al., 2018). In conclusion, further pre-clinical testing in multiple pre-clinical models will be necessary to determine the potency of targeting the DNA damage response in ARIDIA mutant cancers.

ARIDIA deletions were also found to cause a vulnerability to inhibitors of the glutathione synthesis metabolic pathway (Gorrini \& Mak, 2019; Ogiwara et al., 2019). APR-246, a glutathione inhibitor, reduced tumor burden in an Aridla mutant cancer model, but not in an ARIDIA wild-type in vivo model (Ogiwara et al., 2019). Additional experiments also suggested that this pathway may include multiple synthetic lethal options for targeting Aridla mutant cancers. APR-246 has shown promise in p53 deficient acute myeloid leukemia and chronic lymphatic leukemia clinical trials (Deneberg et al., 2016). Glutathione antioxidant synthesis is applicable to many ARID1A depleted cancers, and further testing will be necessary to determine whether the effects are cancer-type dependent or generally apply to ARIDIA mutant cancers.

In summary, the downstream effects of $A R I D I A$ mutations will likely be cancer specific, therefore requiring validation of each ARIDIA mutated cancer type.

Furthermore, combinations of SL-SMIs and chemotherapeutics during pre-clinical testing may provide the best prediction of the efficacy of these new precision medicines in patients.

\section{Cell Identity}

One of the major burdens across cancer genetic and tumorigenesis fields is the lack of identification of the cell of origin in many cancer types. In NBL, cell phenotypes evaluated in clinical and basic research studies of patient tumors settings support the view that this tumor type originates in sympathoadrenal precursors arising from the trunk neural crest cell (NCC) lineage. Decades of developmental studies also support this concept. Therefore, there is consensus in the NBL community that defines this cell type as the most probable cell of origin of NBL. Recent advances in developmental research have focused on thoroughly mapping decision branchpoints and defining gene expression profiles in NCCs with the goal of specifying how cell fate decisions are made (Furlan et al., 2017; Soldatov et al., 2019). These recent studies provide clarity to the regulation of NCC identity during development and will be described in more detail in the discussion section

The nervous system originates in the outermost embryonic layer, the ectoderm, and is spawned from the neural tube (Squire et al., 2003). At this point, the central nervous system is developed to innervate the brain and spinal cord, where branches of neurons connect and communication among the three germ layers begins (Squire et al., 2003). Next, the sympathetic nervous system (SNS) is born from the ectoderm (Squire et al., 2003). The location at the origin of the neural crest specification restricts what fate 
the cells will acquire (Dupin, Creuzet, \& Le Douarin, n.d.). There are four classifications of neural crest cells: cranial, vagal, trunk, and sacral (Squire et al., 2003). Each of these give rise to different categories of cells (Figure 1-1).

At mouse embryonic day 9.5 trunk NCCs positioned ventral to the developing neural tube begin a migratory excursion initiated by a network of transcription factors. The extensive migration of these NCCs result in the contribution to a variety of specialized tissue types throughout the body. These include the heart, cranial facial bones, melanocytes, adrenal chromaffin cells, sympathetic neurons and ganglia (enteric, dorsal root, sympathetic, and parasympathetic) (Chan, Anderson, \& Gonsalvez, 2018; Squire et al., 2003). Trunk NCCs migrate throughout the body and form the SNS and adrenal medulla. NBL arises predominately in the adrenal medulla, but may occur anywhere along the parasympathetic ganglia, demarcating it as a cancer of the developing SNS (Jiang et al., 2013; Schulte \& Eggert, 2015). This review will focus on concepts and cell types that comprise the SNS.

\section{Neural crest cells}

NCCs are found only in vertebrate organisms and migrate extensively during development to give rise to the SNS. They arise at the "crest" of the closing neural tube, ventral to the neural plate, at the border of epidermal ectoderm (Chan et al., 2018). Extrinsic factors such as location of origin, timing of migration, cell-to-cell contact and signaling greatly influence the internal TF cascades that determine each cell's fate. Trunk neural crest cells (NCCs) give rise to the developing SNS, which includes parasympathetic and sympathetic ganglia (Schwann cells), sympathetic neurons, and chromaffin cells of the adrenal medulla (Chan et al., 2018). These motile cells journey throughout the body to eventually connect organs to their host. This connection serves to prepare the body to respond to stimuli through endocrine signaling events of the adrenal glands, resulting in a "fight or flight" response. The first step in the trunk NCC differentiation process, is delamination, then migration to the developing dorsal aorta, next, these cells coalesce, and receive signals that specify them to the sympathetic fate (Etchevers, Dupin, \& Le Douarin, 2019; Huber, 2015; Mirsky et al., 2008). Secreted homing signals such as SDF-1, BMPs, and other cytokines and growth factors in the local environment direct NCCs to their final destinations (Squire et al., 2003). Intracellularly, TF master regulators such as SOX9, HAND2, GATA3, ASCL1, TCF4, SLUG and TWIST1 modulate cellular programs that determine localization and fate. NCCs are highly adaptive, or plastic, requisite to the ability to respond to and thrive in the numerous environmental conditions that must be adapted to during their extensive migratory journey (Squire et al., 2003).

During pre-migration, NCCs morphology is epithelial, with columnar shape and a polar apical top with strong cell-to-cell adhesion junctions (Squire et al., 2003). Furthermore, the cells are multipotent prior to migration (Chan et al., 2018; Soldatov et al., 2019). When the migratory program is initiated, NCCs undergo an epithelial to 


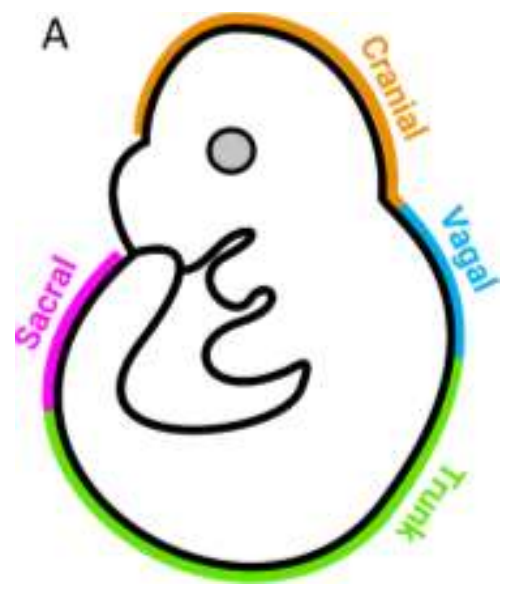

Figure 1-1. Schematic of neural crest origins.

(A) Schematic depicting the locations in the embryo that give rise to different varieties of neural crest. Top to bottom: cranial (orange), vagal (blue), trunk (green), and sacral (magenta). 
mesenchymal transition (EMT), losing cell-to-cell adhesion, adopting a fibroblastic shape and motile behavior (Chan et al., 2018; Squire et al., 2003). During delamination, NCCs begin demonstrating patterns of gene expression that indicate a fate bias (Soldatov et al., 2019). The EMT program is initiated by a combination of epigenetic factors and TFs (Bajpai et al., 2010). In zebrafish embryos, the pBAF complex (containing ARID2) was shown to regulate the initiation of NCC migration, specification, and multipotency through the TFs Twist1, Slug and Sox9 (Bajpai et al., 2010). These experiments connected epigenetic regulators, particularly SWI/SNF, to the regulation of a cascade of TFs involved in NCC EMT. This study also causally identified these TF genes as master regulators of EMT migratory programs in the neural crest. One outstanding question arising from this topic is how deregulation of the EMT migratory program contributes to disease, specifically NBL and other crest-related pathologies.

NBL arises at different stages during early developmental, and presents with many genetic lesions, resulting in substantial intratumoral, and intertumoral heterogeneity (Boeva et al., 2017; Garrett M. Brodeur, 2003; van Groningen et al., 2017). A recent study established that MNA NBL cell lines are addicted to transcription factors that are up-regulated in this cancer sub-type (Durbin et al., 2018). Independent knockdown of the up-regulated transcription factors led to a reduction of colony size in MNA cell lines, but not controls (Durbin et al., 2018). These transcription factors have been linked to cell identity during NCC development, and NBL disease progression. Addition studies have worked to better define the cell identities in NBL tumors. One group worked to classify three cell identities in a panel NBL tumors and cell lines (Boeva et al., 2017). Resulting cell identity groups consisted of (I) noradrenergic, (II) NCC, and (III) mixed cell identities (Boeva et al., 2017). Primary tumor samples were composed of mixed cell identities, meaning both noradrenergic and NCC cells were present (Boeva et al., 2017). They found that the NCC NBL identity was associated with recurrent super enhancers in transcription factors that regulate mesenchymal cell identity (Boeva et al., 2017).

The second study characterized patient NBL tumors by sorting for stemness, then identifying a "super-enhancer-associated transcription factor network" (van Groningen et al., 2017). Two NBL patient samples were sorted by CD133 expression into positive and negative groups and these cells were analyzed by whole-genome sequencing (van Groningen et al., 2017). Two transcription factor gene signatures were developed from the analysis: adrenergic (ADRN) and mesenchymal (MES) (van Groningen et al., 2017). Both signatures included classic NBL markers. ADRN genes included the neuronal signature master regulators DBH, GATA2, GATA3, PHOX2A, and PHOX2B (van Groningen et al., 2017). MES genes included several EMT regulators SNAI2, VIM, FN1, YAP1, SOX9 and PRRX1 (van Groningen et al., 2017). These signatures were consistent with the super-enhancer regulated transcription factors identified previously (Boeva et al., 2017). Furthermore, van Groningen et al. demonstrated that these two cell types could be reprogrammed to interconvert, or trans-differentiate by enforcing an ADRN cell identity to convert to an MES identity when PRRXI expression was enforced (van Groningen et al., 2017). These results suggest that the MES cell type is more immature than the ADRN complement, because the signature overlaps with a NCC EMT signature (van Groningen et al., 2017). This study also suggested that both tumor types originate from an earlier 
stem-like precursor, although this initiating cell has not been identified. Furthermore, these studies point to the importance of the master regulators controlling cell identity and suggest that differential expression of these genes may influence NBL disease progression and treatment outcomes.

\section{Cancer models of initiating oncogenic events}

The NBL field and other pediatric cancer fields establish their findings using classic cancer models often developed by adult cancer specialists. This includes the use of established cancer cell lines, or patient derived material (D. T. W. Jones et al., 2019). Both are extremely powerful tools for high-throughput screening and drug development. However, one weakness of these approaches is the lack of ability to identify and test initiating events underlying the disease of interest. In the approaches listed above, the cancer cells are already transformed, and additionally may have been exposed to chemotherapeutics or other mutating events, complicating the interpretation of existing mutations and how they contribute to the disease (Tsubota \& Kadomatsu, 2018). In NBL, genetically engineered mouse models (GEMMs) have laid a strong foundation for testing possible cancer-initiating genetic events in isolation. One fundamental example of a GEMM model that causally implicated $M Y C N$ as an oncogenic driver of NBL is the mouse model developed by Weiss et al. (Weiss et al., 1997). This mouse model leverages the TH promoter to drive $M Y C N$ expression in sympathetic neurons and is the most widely used mouse model of NBL. However, it is challenging and time intensive to test, screen and capture early transforming events, especially in a variety of combinations using current GEMM approaches. An alternative approach to GEMM models in the NBL field has been to induce NCCs from human stem cells (Huang et al., 2016). Although promising, one limitation of primary cells is the challenge of matching culture conditions to in vivo conditions, especially throughout many stages of developmental changes. Another complication is the limited ability to expand and manipulate primary cells in vitro using current engineering strategies. These challenges led to the development of an additional cancer model that can complement the field's current approaches to solving developmental cancer questions, such as how normal cell identity is transformed into a cancerous cell state.

Neural tube explants have been a classic method in the developmental field, and extensive experimentation has been completed in drosophila, chick embryos, zebrafish, and now mouse models (Squire et al., 2003). Applying the neural tube explant procedure to existing GEMM applications allows GEMM and in vitro modifications to be employed in one cell population while connecting in vivo and in vitro testing.

Previously, our laboratory developed a trunk NCC transformation technique using neural tube explants. In the first iteration of this method, wild-type embryos were generated, and at day 9.5, the embryos were harvested from the uterine horn. Next, the neural tube was resected and placed in culture. Neural crest cells located on the trunk migrated onto the dish for 48 hours before the neural tube was removed. Mycn overexpression was enforced in vitro to recapitulate MNA, and the modified NCCs were 
flank injected back into mice. As expected, Mycn was sufficient to transform the primary NCCs into a tumor that molecularly and pathologically recapitulated human MNA NBL (Olsen et al., 2017).

One trait intrinsic to NCCs is their migratory ability (Chan et al., 2018; Etchevers et al., 2019; Furlan et al., 2017; Squire et al., 2003). NCC migration properties, paired with their progenitor cell plasticity, makes them well suited for studying early stages of SNS development (Etchevers et al., 2019). The NCC model described above provides an advantage to other established models by using NCCs at a stage of development where they have undergone EMT and are primed to receive differentiation signals. Capturing NCCs during this time allowed us to ask the question, what genetic lesions are transformative in progenitor cells during development, and through what mechanisms? The main approach in early experiments with this model has been to model NBL chromosomal alterations and to test whether these abnormalities during early SNS development redirect normal properties of NCCs into an un-regulated cancerous cell. The ability to study the effects of genetic events, one at a time, or in combination, to inject the cells, and generate tumor tissue and tumor derived cell lines (TDCLs) provides a tool that combines developmental and cancer approaches. We have applied traits of NCCs to enable systematic testing of the genetic events that underlie this disease of development.

At embryonic day 9.5 (e9.5), trunk NCCs follow either a dorsal or ventrolateral migration stream (Squire et al., 2003). Between e9.5 and e11.5, mouse trunk NCCs undergo a sequence of binary decisions that result in four different cell identities (Soldatov et al., 2019). NCCs migrate in streams (Furlan et al., 2017). The flow of NCCs continues through cell to cell, tail to head contact that results in a chain-like structure of NCCs that allow other migrating NCCs to use as an internal ladder, or path to follow to the more distant NCC population sites, such as the adrenal medulla (Squire et al., 2003; Furlan et al., 2017). Recently, a landmark paper was published that highlighted how celltype-specific deletions influence adrenal medulla formation (Furlan et al., 2017). Using cell fate mapping techniques in multiple mouse models, NCC chains were found to be necessary for migratory cells to populate the adrenal medulla (Furlan et al., 2017). Furthermore, mutation of the migratory cells also resulted in a lack of adrenal medulla colonization (Furlan et al., 2017). These findings suggest that mutations in multiple cell types that differentiate into the SNS may cause abnormal adrenal medulla pathology (Etchevers et al., 2019; Furlan et al., 2017). However, the experiments presented by Furlan, et al. were focused on developmental questions of SNS development, rather than transformative events during development.

\section{Defining the Critical Questions to Address}

\section{What is the oncogenic action of MYCN?}

In normal development, a pulse of $M Y C N$ expression is necessary for enforced

proliferation of the developing SNS. One outstanding question is how MYCN expression 
transitions from pushing normal proliferation to causing deregulated oncogenic proliferation. Amplification of $M Y C N$ in NBL is an established oncogenic event, but the events that transpire to exert its oncogenic actions are not well understood (Rickman et al., 2018; Zeid et al., 2018). One theory describes $M Y C N$ expression as additive, meaning higher $M Y C N$ expression causes higher expression of its target genes, mostly those that increase proliferation and stemness, therefore leading to uncontrolled growth (Rickman et al., 2018). However, in 2017 an article was published that contributed an understanding of $M Y C N$ that was crucial to addressing part of this question. MYCN has an established role in binding promoters with canonical E-boxes. However, approximately 50 percent of its binding sites are at non canonical E-boxes (Rickman et al., 2018). In NBL, MYCN was shown to bind to the promoters of genes that are part of the NBL gene signature, such as Hand2, Twist1, Phox2b, Ascl1, and Sox9 (van Groningen et al., 2017; Zeid et al., 2018). This emerging evidence suggests that the aberrant binding of MYCN at enhancers may be a potent part of how MYCN exerts its oncogenic activity (Rickman et al., 2018; Zeid et al., 2018). The most current literature draws a picture depicting normal $M Y C N$ binding at open promoters with conserved E-box sites, but when amplified, invading the open enhancers of lineage factors, leading to tissue specific gene expression that is associated with oncogenic behavior, or "oncogenic enhancer-driven transcription" (Zeid, 2018, Nat Gen).

\section{Does loss of Aridla result in a synergistic relationship with $M Y C N$ ?}

Aridla has been implicated as a TSG in numerous cancer types and has additional roles in developmental models. During normal conditions, ARIDIA has been identified as necessary for the G2-S checkpoint, is present in the BAF complex, and has been shown to regulate lineage commitment during hematopoiesis (Han et al., 2019; Kadoch et al., 2013; Shen et al., 2015). One developmental study determined that complete ablation of Aridla resulted in a fewer SNS TH+ neurons in comparison to heterozygous Aridla deletion (Gao et al., 2008). A study of adult liver cancer identified overexpression of $A R I D 1 A$ in primary tumor tissue, and loss of ARIDIA in metastatic clones (Sun, Wang, et al., 2017). Sun, et al. interpreted this result as an indication that $A R I D 1 A$ is a suppressor of metastasis, but not initiation in their model (Sun, Wang, et al., 2017; Yoshida, Sokoloff, Welch, \& Rinker-Schaeffer, 2000). Studies involving ARIDIA suggest that a combination of methods and tools from multiple disciples will be necessary to further our understanding of $A R I D 1 A$. One question that is raised when considering whether ARID1 A has a TSG role in NBL, is what is the TSG function of ARID1A in this cancer? Considering that this a disease of development, we hypothesized that ARIDIA deletion can contribute to the formation of mutant NCCs by altering normal differentiation patterns and resulting in transformation into NBL. Approaching this question requires tools that span the boundary of in vivo and in vitro studies and allow a model that enables the transformation of primary cells. To accomplish this, techniques that bridge cancer biology and developmental biology fields were modified and applied to this disease context. The study of the ARIDIA TSG in a dose dependent, tissue specific, and an NBL sub-type specific cell state will allow the connection between the oncogenic effects of MYCN and epigenetic deregulation due to Aridla loss to be tested. Developing an 
approach to model and combine possible synergistic NBL initiating events is critical for connecting the genetic lesions that underlie this aggressive and lethal sub-type of NBL to NCC deregulation and transformation. 


\title{
CHAPTER 2. METHODOLOGY
}

\author{
Neural Crest Cell Methods
}

\section{Neural crest cell isolation}

Neural crest cells were derived from Aridla floxed mice (Gao et al., 2008). First, two to three female mice were placed in individual cages, and in the afternoon of day 0 , one male mouse was place in each cage. The following morning, and day 0.5 , the males were all removed, and female mice were checked for plugs, and weighed with weights and plugged status documented for each female. Plugged females were condensed into the fewest possible cages, and breeding cards were updated. On day 8.5, in the afternoon, the females are weighed again, and females gaining $<1 \mathrm{~g}$ were excluded from further experimentation and added back to their original colony for monitoring. Females gaining $\geq 1 \mathrm{~g}$ were palpated, and pregnant females were separated for embryo extraction.

The next morning on day 9.5, instruments and dissecting equipment were sterilized for 15 minutes with 70\% ethanol, and fresh Chemically Defined Medium (CDM) was prepared (Fukuta et al., 2014). Embryos were dissected from each pregnant female and placed in sterile PBS on ice. Embryos were kept on ice, and two at a time were moved to the dissection scope for dissection. Embryonic sacs were removed, one by one using sterile sharp tweezers, and extracted whole embryos were placed in a $60 \mathrm{~mm}$ rubber coated petri dish in sterile PBS using a blunted pipette. Once all embryos were extracted, the neural tubes were dissected. Insect dissection pins were used to pierce the eye and tail tip and extend the embryo, next a scalpel was used to separate the neural tube and include somites $23-19$. The cuts were made caudal to the heart, and $.5 \mathrm{~cm}$ from the tail's end. Each neural tube was extracted, and five were placed in each well of a 24 -well sterile tissue culture treated plate.

Next,1x collagenase and dispase (Sigma, 10269638001), was used to gently dissociate the neural tube tissue during five minutes of titeration with a Pasteur pipette. The dissociated neural tubes were rinsed in PBS and moved into individual wells containing $50 \mu \mathrm{l}$ of CDM. Next, a $20 \mu \mathrm{l}$ pipette was used to transfer the embryo and $\sim 10$ $\mu 1$ of media to a fibronectin-coated 96-well tissue culture treated plate containing $100 \mu 1$ of CDM. In 4hrs, the neural tubes were observed for attachment to the plate, and an additional $100 \mu 1$ of CDM was added. At 48 hours following plating, the neural tubes and tissue were removed from the plate and collected into individual sterile Eppendorf tubes, leaving behind the attached populations of NCCs. At this point, additional media may be added. The harvested neural tube tissue was subjected to genotyping for the floxed Aridl $a$ allele. The NCCs were monitored for confluency, and all of the cells were accutased, alike genotypes were combined then plated in an appropriate-sized dish (500,000 cells $/ 10 \mathrm{~cm}$ fibronectin coated tissue culture petri dish). Media must be refreshed every three days, and cell stocks need to be frozen as the highest priority. Next, the cells were expanded and modified for experiments, and mouse injections. 


\section{Modification of neural crest cells}

Neural crest cells were cultured in CDM, genotyped, pooled by genotype, and transduced with Adeno-Empty-iGFP or Adeno-CRE-iGFP virus at MOI of 3 or 11, respectively (virus purchased from University of Iowa viral vector core Ad-GFP \# VVCU of Iowa-4, Ad5CMVeGFP; Ad-Cre-GFP \#VVC-U of Iowa-1174, Ad5CMVCreeGFP). Cells were sorted at 72 hours post transduction for GFP expression, and resulting Aridla $^{+/ \text {flox }}$, Aridla ${ }^{+/-}$, Aridla $a^{\text {flox/fox }}$, or Aridla $a^{-/-}$cells were cultured up to 12 passages. Neural crest cell derivation was previously described (Olsen et al., 2017).

\section{Tumor measurement cutoffs and statistics}

Tumor measurements began when tumors were approximately $5 \mathrm{~mm}$ x $5 \mathrm{~mm}$. Tumor initiation was considered complete at $300 \mathrm{~mm}^{3}$, and survival end points were measured at $1500 \mathrm{~mm}^{3}$. Animals were humanely euthanized according to SJCRH IACUUC regulation standards. Study measurements were reported at $300 \mathrm{~mm}^{3}$ and 1500 $\mathrm{mm}^{3}$ and statistics were calculated at these time points.

\section{Tumor studies in SCID mice}

The following primary cell isolations were used for the experiments in SCID mice. Aridla $a^{+/ \text {flox }}$ (WT), Aridla $a^{+/-}$(HET) (isolated 10/15/2017 plate 3), Aridla flox/fox $^{\text {(WT }}$ (WT), or Aridla $a^{-/-}$(KO) (isolated 8/15/2016). The primary NCCs were transduced for 24 hours with $M Y C N$-iRES-GFP virus (viral prep10/29/2013; Addgene \#35394) at an MOI of 1 . Cells at $85 \%$ confluence were resuspended using accutase and counted, then pelleted and resuspended in 1:1 PBS and Matrigel. Female severely compromised immunodeficient (SCID) mice from Taconic were purchased at 6 weeks of age and quarantined for 1 week before subcutaneous injections (C.B-Igh-1b/IcrTac-Prkdcscid). Mice were anesthetized with isofluorane for 5 minutes and injected on the right flank subcutaneously with a two-fold titration beginning with $6 \times 10^{6}$ cells, down to $0.75 \times 10^{6}$ cells per mouse.

\section{Tumor studies in nude mice}

Female homozygous athymic nude mice (Crl:NU-Foxn1nu) were purchased from Jackson Laboratories at 6 weeks of age (Cat \#088), held for at least one week before injecting with Aridla $a^{+/ \text {flox }}$, Aridla $a^{+/-}$, Arid $1 a^{\text {flox/fox }}$, or Arid $1 a^{-/-}$primary neural crest cells transduced for 24 hours with $M Y C N$-iRES-RFP virus at an MOI of 3 (Addgene \#35393). Cells at $85 \%$ confluence were resuspended using accutase and counted, then pelleted and resuspended in 1:1 PBS and Matrigel. Mice were anesthetized with isofluorane for 5 minutes and injected on the right flank subcutaneously with $1.5 \times 10^{6}$ cells per mouse. 


\section{Tumor harvest procedure}

In all animal studies, tumors were measured every other day, beginning at 200 $\mathrm{mm} 3$, and mice were euthanized when tumors reached $2000 \mathrm{~mm} 3$. All animal studies were conducted in compliance with protocols approved by the St Jude Children's Hospital Institutional Animal Care and Use Committee. Tumors were harvested immediately following euthanasia for all experiments. At harvest, one cross section was placed in 4\% neutral buffered paraformaldehyde for immunohistochemistry analysis. The remaining tumor was sectioned into $1 \mathrm{~mm}$ pieces in a dish containing chemically defined media, and distributed into tubes for protein, RNA, and DNA processing before being flash frozen in liquid nitrogen, and was also distributed into tubes containing freezing medium $(\mathrm{CDM}+10 \% \mathrm{DMSO})$ before subsequent cryopreservation for viable cell storage.

\section{Derivation of cell lines from fresh tumor sample}

To derive tumor cell lines, the remaining media from the harvest was collected, and live cells were pelleted, and resuspended in red blood cell lysis buffer for 10 minutes with brief vortexing at 0,5 , and 10 minutes. Cells were re-pelleted, and resuspended in $\mathrm{CDM}+20 \% \mathrm{FBS}$, plated on fibronectin coated $10 \mathrm{~cm}$ dishes, and maintained at $37^{\circ} \mathrm{C} 5 \%$ $\mathrm{CO}_{2}$ with $5 \mathrm{~mL}$ of media added the following day, and changed the second day. Media patterns (fresh media on day 0 , add $5 \mathrm{~mL}$ of media on day 1 , full media change on day 2) were followed until samples were confluent and frozen in stocks.

\section{Neuroblastoma Cell Lines}

SK-N-AS, SY5Y, SK-N-SH, SK-N-DZ, Kelly, IMR-32, and SK-N-BE2 were purchased from American Type Culture Collection (ATCC) and cultured according to manufacture instructions. A table listing a summary of genetic information from Harenza, et al. for MYCN and 1p36 status in common neuroblastoma cell lines are listed in Appendix A Table A-1 (Harenza et al., 2017).

\section{Pathology and IHC Analyses}

Tumors were fixed in 10\% neutral buffered formalin, embedded in paraffin, sectioned at $4 \mu \mathrm{m}$, stained with hematoxylin and eosin (HE). All formalin-fixed, paraffin-embedded (FFPE) tissues were sectioned at $4 \mu \mathrm{m}$, mounted on positive charged glass slides (Superfrost Plus; Thermo Fisher Scientific, Waltham, MA), and dried at $60^{\circ} \mathrm{C}$ for 20 minutes. The IHC procedures and antibodies used for mouse tissue to detect protein markers are listed in Table A-2. All HEs and IHCs were reviewed by light microscopy and interpreted by a board-certified veterinary pathologist Dr. Heather Tillman (HT). IHCs were scanned at a 20x (objective lens) magnification using an Aperio ScanScope XT scanner. Immunoreactivity was quantified as a weighted IHC 
score (0-300) using a modified version of the Color Deconvolution algorithm and ImageScope software (Leica Biosystems).

\section{Immunoblotting}

Detailed descriptions that are project-specific may be found in Chapters 3 and 4. In summary, samples were collected either by centrifugation for five minutes at 3,000 $\mathrm{RCF}$ at $4^{\circ} \mathrm{C}$, were resuspending in PBS, then centrifugation was repeated. PBS was aspirated, and pellets were frozen until further processing. Otherwise, samples were collected on ice by washing 1x with PBS, then adding RIPA buffer (Thermo Scientific, PI89900) and 2x Halt Protease Phosphatase inhibitor. Samples were scraped into a tube, vortexed for 10 seconds and lysed for 20 minutes rotating at $4^{\circ} \mathrm{C}$. Samples were sonicated for 10 second intervals totaling 30 seconds, then lysates were cleared by centrifugation. Samples were incubated for 30 minutes in 1x SDS-loading buffer with 9\% BME and $50 \mathrm{ug}$ of total lysate was separated by gel electrophoresis on $4-20 \%$ tris-glycine gels (BioRad \#4561094) at 300V for 25 minutes. Samples were transferred according to protein size. PVDF Membranes were blocked for 1 hour at room temperature (RT) in PBS .1\% Tween 20 (PBST) $/ 5 \%$ (w/v) milk. Membranes were probed with primary antibodies incubated $\mathrm{o} / \mathrm{n}$ at $4^{\circ} \mathrm{C}$ with gentle shaking. Next, membranes were washed with PBST and incubated with secondary antibody (1:4000, Cell Signaling Technologies) in PBST $/ 5 \%(w / v)$ milk for 1 hour at RT. Membranes were washed in PBST and protein signal was detected using chemiluminescent substrate (Genesee Scientific \#20-302). Antibody concentrations and protein sizes reference Table A-3.

\section{RNA-Sequencing}

Tumor tissues were flash frozen in liquid nitrogen and stored at $-80^{\circ} \mathrm{C}$. The Qiagen RNeasy kit was used to purify RNA from tissue samples. Approximately $15 \mathrm{ug}$ of tumor tissue was resected and homogenized for one minute in Qiagen buffer RLT plus $\beta \mathrm{ME}$. Next, $800 \mathrm{ul}$ were removed, and samples were spun down for 5 minutes at 3000 rpm. Supernatant was removed, and $600 \mathrm{ul}$ was passed through a Qiashredder column. Each tumor type was represented in biological quadruplicates. Samples were eluted in 50 ul of RNAse/DNAse free water, and $500 \mathrm{ng} /$ sample was given to the Hartwell Center at St Jude CRH for an RNA purity check, and RNA sequencing analysis.

\section{Processing of RNA-sequencing results}

Bioinformatics data processing and analysis was performed by Jeremiah Holt. FASTQ files were aligned to the mm10 genome and transcript level estimates were generated with counts in units of transcripts per million (TPM). Next, transcripts with less than ten total counts across all samples were filtered, and the remaining genes were normalized and $\log 2$ transformed using the regularized log transformation (rlog) function in the DESeq2 R package (M. I. Love, Huber, \& Anders, 2014). Samples were then 
averaged to give mean expression values for the remaining genes for each experimental group. DESeq2 and all downstream analyses were conducted using $\mathrm{R}$ version 3.6.1 ( $\mathrm{R}$ Core Team, 2019).

\section{Differential gene expression analysis}

The DESeq2 package was employed again to analyze genes that were differentially expressed between sample groups. DEGs were generated based on three comparisons of experimental groups: HET vs WT1, KO vs WT2, and HET vs KO. Log2 fold changes and adjusted P-values (Benjamini-Hochberg correction) were then used for downstream analyses.

\section{Consensus clustering and unsupervised analysis}

For unsupervised clustering of the normalized gene expression data, we first limited the gene set to only include those that were most variable with a median absolute deviation (MAD) greater than the 75th percentile. Next, ConcensusClusterPlus was implemented using the hierarchical clustering method with average linkage and one minus the Pearson's correlation coefficient as the distance measure, and $\mathrm{k}=2$ clusters was used for downstream analysis (Wilkerson \& Hayes, 2010).

\section{Supervised analyses}

A supervised approach was employed using previously reported gene sets characterizing genetic signatures in neuroblastoma. The first used a predictor based on the MES and ADRN gene sets generated from human neuroblastoma cells (van Groningen et al., 2017). For the purposes of applying this predictor to a murine model, we used only the genes from the MES and ADRN sets that had orthologs in the murine genome. Next, we applied an integrative correlations method to select only those genes which displayed similar expression patterns between the human and mouse models (Parmigiani, Garrett-Mayer, Anbazhagan, \& Gabrielson, 2004). Only the genes with positive integrative correlation coefficients (ICC) were used for the mouse predictor, and three genes were omitted due to the MES and ADRN labels having inconsistent results with the human predictor. A gene set enrichment analysis was then conducted for the new MES and ADRN gene signatures derived from the murine predictor (Olsson et al., 2011; Subramanian et al., 2005). The second supervised approach used a mesenchymal gene signature and a list of neuroblastoma master regulator genes from (Furlan et al., 2017) to visualize differences in gene expression between sample groups based on previously characterized genes. 


\section{CHAPTER 3. ARIDIA IS A CRITICAL TUMOR SUPPRESSOR GENE IN 1 P36 DELETED, MYCN AMPLIFIED NEUROBLASTOMA}

\section{Introduction}

Neuroblastoma is the most common extracranial pediatric solid tumor, and accounts for 15 percent of all pediatric cancer deaths (Maris, 2010; Maris, Hogarty, Bagatell, Cohn, \& Susan, 2007). High-risk (HR) NBL patient survival remains poor, between 30-50\% (Cohn et al., 2009; Maris, 2010). These poor outcomes are a result of multiple tumor characteristics including heterogeneity that is characterized by a multitude of chromosomal gains, translocations, and deletions that result in aggressive and difficult to treat disease. Fifteen to thirty three percent of NBL patients are affected by one subtype of high risk NBL that is driven by the oncogenic event $M Y C N$ amplification (MNA) (Garrett M. Brodeur, 2003; Davidoff, 2012; Jiang et al., 2013; Nakagawara et al., 2018; Rickman et al., 2018)). The majority of HR MNA cases include 1 p36 loss of heterozygosity ( $\mathrm{LOH})$, and this genetic lesion is found in $35 \%$ of overall NBL patients (Garrett M. Brodeur, 2003). Due to the high rates of 1p36 LOH in NBL patients, LOH at $1 \mathrm{p} 36$ is expected to be an initiating event in MNA NBL. Furthermore, patients with 1p36 $\mathrm{LOH}$ and MNA have poor overall survival (46\%) relative to patients with $1 \mathrm{p} 36$ deletions alone (85\%) (Attiyeh et al., 2005). Decades of sequencing studies have led to a list of putative 1p36 tumor suppressor genes (TSGs) including CASZ1, P75, CAMTA1, and CHD5 (Garrett M. Brodeur, 2003). However, despite mapping efforts, the critical 1p36 TSGs that collaborate with $M Y C N$ in NBL initiation remain unidentified.

One issue complicating the identification of $1 \mathrm{p} 36$ TSGs in NBL cases with MNA and $1 \mathrm{p} 36$ heterozygous deletions, is that point mutations have rarely been identified on the second allele for any putative TSGs. This suggests that loss of a single allele of the $1 \mathrm{p} 36$ TSG candidate is sufficient to promote tumor initiation or progression (Garrett M. Brodeur, 2003; Caron et al., 1995; Jiang et al., 2013). Therefore, we predict that the 1p36 TSG demonstrates haploinsufficiency. In 2013, following whole genome and whole exome sequencing analysis of a large NBL patient cohort, Sausen et al discovered point mutations in the TSG Aridla, which highlighted Aridla as a putative 1p36 TSG (Sausen et al., 2013). Recently, an additional study confirmed Aridla as a 1p36 gene of interest in $M Y C N$-driven NBL(García-López et al., 2020). Garcia-Lopez, et al engineered large $1 \mathrm{p} 36$ deletions in combination with MNA, and identified significant deletions in Arid1a were enriched in the final tumors of a tumor evolution study (García-López et al., 2020).

Aridla is a TSG candidate that is frequently mutated across 25 adult and pediatric cancer types including NBL (R. C. Wu et al., 2014). Aridla is the DNA-binding subunit of the SWItch/Sucrose Non-Fermentable (SWI/SNF) chromatin remodeling complex and in cancer, is the most commonly mutated member of this multi-subunit complex (S. Jones et al., 2012; Kadoch et al., 2013; Wiegand et al., 2010). Studies completed in adult cancer models have provided substantial evidence that Aridla is a context specific TSG and a regulator of cell renewal and identity (Flavahan et al., 2017; Huether et al., 2014; Mathur, 2018; Sun, Chuang, et al., 2017). Epigenetic remodelers are the most frequently mutated 
genes in pediatric cancers, however, pediatric cancer studies focused on Aridla mutations have not been explored. The growing evidence of Aridla mutations as a TSG in many cancer types highlight the necessity of causal Aridla studies in the correct cellular context. Therefore, it is necessary to characterize Aridla mutations in a pediatric cancer model of $M Y C N$-driven NBL.

NBL is a disease of development that arises from trunk neural crest cells (NCCs) (Jiang et al., 2013). Trunk NCCs are a highly migratory population of progenitor cells of the developing sympathetic nervous system (SNS). During normal development NCCs undergo an epithelial to mesenchymal transition (EMT), migrate, then specify into the SNS (Furlan et al., 2017; Jiang et al., 2013; Kuo \& Erickson, 2010). During development, NCCs transiently express high levels of N-Myc (day 9.5), before gradual downregulation, differentiation, and quiescence (Jiang et al., 2013; Zimmerman et al., 1986). Aridla is also expressed at day 9.5 NCCs, (Chandler \& Magnuson, 2016), and is necessary for proper development of NCC derived cell types (Chandler \& Magnuson, 2016; Gao et al., 2008). Taken together, results suggest that Aridla deletions are expected to deregulate enhancers and subsequently disrupt normal lineage commitment. The proposed mechanism of Arid1a mutation mediated oncogenic action is via a block development, expected to result in a highly proliferative population of mutant progenitor cells (Eroglu et al., 2014; Mathur, 2018; B. G. Wilson et al., 2010; M. R. Wilson et al., 2019). The regulated expression of Aridla and MYCN have been validated independently and both were determined as necessary for lineage commitment of NCCs. However, one question that remains is whether Aridla deletions paired with $M Y C N$ OE confers a tumorigenic advantage during NBL initiation. To provide the appropriate modeling of this combination of mutations, it is necessary to employ both cancer and developmental approaches.

The body of work presented in this study aimed to combine foundational tools of both developmental and cancer biology into a novel mouse model of pediatric cancer. This study sought to determine if Aridla loss is a driver of NCC transformation that collaborates with $M Y C N$ overexpression (OE) in NBL tumorigenesis. To address this question, we generated primary trunk NCCs, introduced Aridla heterozygous or homozygous deletions, then enforced $M Y C N$ OE to generate NBL as previously described (García-López et al., 2020; Olsen et al., 2017). Next, these modified cells were subcutaneously injected into immunocompromised mice and tumorigenesis was evaluated. Here, we systematically test Aridla wild-type, heterozygous, and knockout (WT, HET, KO) doses in NCCs combined with MYCN OE. Next, tumorigenesis, tumor lineage commitment, and primary cell differentiation were evaluated. Finally, multiple sequencing techniques were used to cross-validate the genes that were mutated or aberrantly regulated as a consequence of Aridla and MYCN genetic lesions. Implementation of a developmental cancer model, downstream sequencing analysis, expression analysis of primary cells and tumors, and functional studies before and after transformation resulted in the identification of disrupted transcription factor signaling circuits that are critical to the control of EMT and cell identity. 


\section{Materials and Methods}

\section{Cell culture}

Neural crest cells were derived from Aridla floxed mice, bred to generate day 9.5 embryos before neural tube excision and culture. Neural crest cells were cultured in chemically defined media, genotyped, pooled by genotype, and transduced with EmptyiGFP or CRE-iGFP virus at MOI of 3 or 11, respectively. (Virus purchased from University of Iowa viral vector core Ad-GFP \# VVC-U of Iowa-4, Ad5CMVeGFP; AdCre-GFP \#VVC-U of Iowa-1174, Ad5CMVCre-eGFP). Cells were sorted at 72 hours

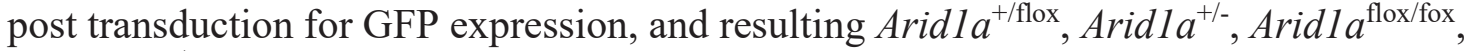

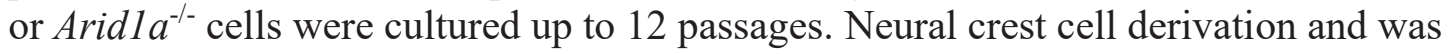
previously described (Olsen, 2017, Oncogene), and enforced MYCN expression was previously described (Olsen, 2017, Oncogene; Garcia-Lopez, 2020, Cell Reports).

\section{Tumor studies in SCID and nude mice}

Aridla $a^{+/ \text {flox }}$, Aridla $^{+/-}$, Aridla $a^{\text {flox/fox }}$, or Arid $1 a^{-/-}$primary neural crest cells transduced for 24 hours with $M Y C N$-GFP virus at an MOI of 1 . Cells at $85 \%$ confluence were resuspended using accutase and counted, then pelleted and resuspended in 1:1 PBS and Matrigel. Female severely compromised immunodeficient (SCID) mice from Taconic were purchased at 6 weeks of age and quarantined for 1 week before subcutaneous injections (C.B-Igh-1b/IcrTac-Prkdcscid). Mice were anesthetized with isofluorane for 5 minute and injected on the right flank subcutaneously with a two fold titration beginning with $6 \times 10^{6}$ cells, down to $0.75 \times 10^{6}$ cells per mouse.

Female homozygous athymic nude mice (Crl:NU-Foxn1nu) were purchased from Jackson Laboratories at 6 weeks of age (Cat \#088), held for at least one week before injecting with Aridla $a^{+/ \text {flox }}$, Aridla $a^{+/-}$, Arid $1 a^{\text {flox/fox }}$, or Arid $1 a^{-/-}$primary neural crest cells transduced for 24 hours with $M Y C N$-RFP virus at an MOI of 3 (Addgene \#35393). Cells at $85 \%$ confluence were resuspended using accutase and counted, then pelleted and resuspended in 1:1 PBS and Matrigel. Mice were anesthetized with isofluorane for 5 minutes, and injected on the right flank subcutaneously with $1.5 \times 10^{6}$ cells per mouse.

Tumors were harvested immediately following euthanasia. At harvest, one cross section was placed in 4\% neutral buffered paraformaldehyde for immunohistochemistry analysis. The remaining tumor was sectioned into $1 \mathrm{~mm}$ pieces in a dish containing chemically defined media, and distributed into tubes for protein, RNA, and DNA processing before being flash frozen in liquid nitrogen, and was also distributed into tubes containing freezing medium (CDM $+10 \% \mathrm{DMSO})$ before subsequent cryopreservation for viable cell storage.

To derive tumor cell lines, the remaining media from the harvest was collected, and live cells were pelleted, and resuspended in red blood cell lysis buffer for 10 minutes. 
Cells were re-pelleted, and resuspended in CDM $+20 \% \mathrm{FBS}$, plated on fibronectin coated $10 \mathrm{~cm}$ dishes, and maintained at $37^{\circ} \mathrm{C} 5 \% \mathrm{CO} 2$ with media added the following day, and changed the third day.

In all animal studies, tumors were measured every other day, beginning at 200 $\mathrm{mm} 3$, and mice were euthanized when tumors reached $2000 \mathrm{~mm}^{3}$. All animal studies were conducted in compliance with protocols approved by the St Jude Children's Hospital Institutional Animal Care and Use Committee.

\section{Immunofluorescence}

Primary neural crest cells were cultured in chemically defined media, and plated in fibronectin coated 8-well chamber slides (ibidi cat \#80826) at confluence. The next day, media was aspirated, and cells were wasted with PBS. Neuronal differentiation media was added and refreshed every three days for seven days. The differentiated samples were rinsed 1x with PBS, and 4\% neural buffered paraformaldehyde was added for 10 minutes. Samples were then rinsed with PBS before ice cold $100 \%$ methanol was applied for 30 minutes. Samples were briefly rinsed with PBST before a 10 minute incubation with Triton-X and a 30 minute block in 5\% donkey serum. Primary antibodies were diluted in 5\% donkey serum, Aridla (1:500 CST \#12354) and MAP2 (1:2000 abcam ab5392) and incubated overnight at $4^{\circ} \mathrm{C}$. Wells were washed $1 \mathrm{x}$ with PBST before adding Alexa 555 anti-rabbit and Alexa 488 anti-chicken conjugated secondaries (both at 1:1000 invitrogen A32794, A-11039) at room temperature for 1 hour. Secondary antibodies were removed and wells were washed $3 \mathrm{x}$ with PBST before adding DAPI (0.7 ug/ul) in PBS for 10 minutes, and mounting in PPD mounting media overlayed with mineral oil. Samples were analyzed under a fluorescent microscope, and at least 300 cells were assayed per sample in each experiment.

\section{Immunoblotting}

Samples were collected on ice by washing 1x with PBS, then adding RIPA buffer (Thermo Scientific, PI89900) and 2x Halt Protease Phosphatase inhibitor. Samples were scraped into a tube, vortexed for 10 seconds and lysed for 20 minutes rotating at $4{ }^{\circ} \mathrm{C}$. Samples were sonicated for 10 second intervals totaling 30 seconds, then lysates were cleared by centrifugation. Samples were incubated for 30 minutes in 1x SDS-loading buffer with 9\% BME and 50 ug of total lysate was separated by gel electrophoresis on 420\% tris-glycine gels (BioRad \#4561094) at 300V for 25 minutes. Samples were transferred according to protein size. PVDF membranes were blocked for 1 hour at room temperature (RT) in PBS .1\% Tween 20 (PBST) $/ 5 \%$ (w/v) milk. Membranes were probed with primary antibodies incubated $\mathrm{o} / \mathrm{n}$ at $4{ }^{\circ} \mathrm{C}$ with gentle shaking. Next, membranes were washed with PBST and incubated with secondary antibody (1:4000, Cell Signaling Technologies) in PBST/5\% (w/v) milk for 1 hour at RT. Membranes were washed in PBST and protein signal was detected using chemiluminescent substrate (Genesee Scientific \#20-302). Antibody concentrations and protein sizes reference Table A-3. 


\section{Immunohistochemical staining and analysis}

Samples were processed according to St Jude histology core protocol and analyzed by board certified veterinary pathologist (HT) using color deconvolution (Aperio sofware) described below:

Tumors were fixed in 10\% neutral buffered formalin, embedded in paraffin, sectioned at $4 \mu \mathrm{m}$, stained with hematoxylin and eosin (HE). All formalin-fixed, paraffin-embedded (FFPE) tissues were sectioned at $4 \mu \mathrm{m}$, mounted on positive charged glass slides (Superfrost Plus; Thermo Fisher Scientific, Waltham, MA), and dried at $60^{\circ} \mathrm{C}$ for 20 minutes. The IHC procedures and antibodies used for mouse tissue to detect protein markers are listed in Table A-2. All HEs and IHCs were reviewed by light microscopy and interpreted by a board-certified veterinary pathologist (HT). IHCs were scanned at a 20x (objective lens) magnification using an Aperio ScanScope XT scanner. Immunoreactivity was quantified as a weighted IHC score (0-300) using a modified version of the Color Deconvolution algorithm and ImageScope software (Leica Biosystems).

\section{Migration assays}

Tumor derived cell lines were cultured in chemically defined media, accutased, counted, and resuspended at $1 \times 10^{6}$ cells per $\mathrm{mL}$. The experiment was performed according to the manufacturer's protocol, and $300 \mathrm{ul}$ of the suspension was plated per boyden chamber. In the well, $500 \mu \mathrm{l}$ of $\mathrm{CD}$ media containing $10 \%$ fetal bovine serum was added. Samples were incubated at $37^{\circ} \mathrm{C} 5 \% \mathrm{CO} 2$ for 24 hours, then analyzed by crystal violet staining followed by colorimetric analysis according to manufacturer protocol (Cell biolabs \#CBA-100).

\section{RNA-sequencing sample processing}

Tumor tissues were flash frozen in liquid nitrogen and stored at -80 . The Qiagen RNeasy kit was used to purify RNA from tissue samples. Approximately 15 ug of tumor tissue was resected, and homogenized for one minute in Quiagen buffer RLT plus $\beta \mathrm{ME}$. Next, 800 ul were removed, and samples were spun down for 5 minutes at $3000 \mathrm{rpm}$. Supernatant was removed, and 600 ul was passed through a Qiashredder column. Each tumor type was represented in biological quadruplicates. Samples were eluted in $50 \mathrm{ul}$ of RNAse/DNAse free water, and $500 \mathrm{ng} /$ sample was given to the Hartwell Center at St Jude CRH for an RNA purity check, and RNA sequencing analysis. 


\section{Processing of RNA-sequencing results}

FASTQ files were aligned to the mm10 genome and transcript level estimates were generated with counts in units of transcripts per million (TPM). Next, transcripts with less than ten total counts across all samples were filtered, and the remaining genes were normalized and $\log 2$ transformed using the regularized log transformation (rlog) function in the DESeq2 R package (M. I. Love et al., 2014). Samples were then averaged to give mean expression values for the remaining genes for each experimental group. DESeq2 and all downstream analyses were conducted using $\mathrm{R}$ version 3.6.1 (R Core Team, 2019).

\section{Differential gene expression analysis}

The DESeq2 package was employed again to analyze genes that were differentially expressed between sample groups. DEGs were generated based on three comparisons of experimental groups: HET vs WT1, KO vs WT2, and HET vs KO. Log2 fold changes and adjusted P-values (Benjamini-Hochberg correction) were then used for downstream analyses.

\section{Consensus clustering and unsupervised analysis}

For unsupervised clustering of the normalized gene expression data, we first limited the gene set to only include those that were most variable with a median absolute deviation (MAD) greater than the 75th percentile. Next, ConcensusClusterPlus was implemented using the hierarchical clustering method with average linkage and one minus the Pearson's correlation coefficient as the distance measure, and $\mathrm{k}=2$ clusters was used for downstream analysis (Wilkerson \& Hayes, 2010).

\section{Supervised analyses}

A supervised approach was employed using previously reported gene sets characterizing genetic signatures in neuroblastoma. The first used a predictor based on the MES and ADRN gene sets generated from human neuroblastoma cells (van Groningen et al., 2017). For the purposes of applying this predictor to a murine model, we used only the genes from the MES and ADRN sets that had orthologs in the murine genome. Next, we applied an integrative correlations method to select only those genes which displayed similar expression patterns between the human and mouse models (Parmigiani et al., 2004). Only the genes with positive integrative correlation coefficients (ICC) were used for the mouse predictor, and three genes were omitted due to the MES and ADRN labels having inconsistent results with the human predictor. A gene set enrichment analysis was then conducted for the new MES and ADRN gene signatures derived from the murine predictor (Olsson et al., 2011; Subramanian et al., 2005). The second supervised approach used a mesenchymal gene signature and a list of 
neuroblastoma master regulator genes from (Furlan et al., 2017) to visualize differences in gene expression between sample groups based on previously characterized genes.

\section{Results}

\section{Haploinsufficient Aridla is a driver mutation that collaborates with $M Y C N$ in NBL tumorigenesis}

Aridla mutations in a variety of cancers suggest that it is haploinsufficient in some contexts and homozygous in others (J. N. Wu \& Roberts, 2013). 1p36 LOH can occur in patients without $M Y C N$ amplification, however, $M Y C N$ amplification is rarely present without 1p36 LOH (Garrett M. Brodeur, 2003; Jiang et al., 2013). This suggests 1p36 LOH may provide an environment for MYCN amplification (Jiang et al., 2013). Our laboratory previously identified Aridla as potential $M Y C N$-driven NBL TSG using CRISPR to screen for candidates that increase tumorigenesis in combination with $M Y C N$ overexpression (García-López et al., 2020). As reported previously, Aridla deletion alone does not cause tumorigenesis in NCCs (García-López et al., 2020). To validate and explore the role of Aridla deletions in $M Y C N$-driven NBL in detail, we employed the most published Aridla mouse model which contains floxed sites at exon 8 that cause nonsense-mediated mRNA decay as a result of CRE-mediated excision (Gao et al., 2008; Sun, Chuang, et al., 2017; Sun, Wang, et al., 2017; S. C. Wang et al., 2019). Aridla $a^{\mathrm{Fl} / \mathrm{Wt}}$ and $A$ rid $1 a^{\mathrm{Fl} / \mathrm{Fl}}$ mice were used to generate NCCs according to a previously described method (Olsen et al., 2017). NCCs were pooled by genotype, and floxed Aridla sites were deleted before overexpressing $M Y C N$. This approach generated two matched sets of primary NCCs: 1). Aridla $a^{\mathrm{F} / \mathrm{Wt}}+M Y C N$ (WT1) and Aridl $a^{-/ \mathrm{Wt}}+M Y C N(\mathrm{HET})$, or 2). Arid1 $a^{\mathrm{Fl} / \mathrm{Fl}}+M Y C N(\mathrm{WT} 2)$ and Aridl $a^{-/-}+M Y C N(\mathrm{KO})$ (Figures 3-1A and B-1). To determine whether Aridla deletion and MYCN OE recapitulated the synergy expected from a 1p36 LOH TSG, we evaluated the kinetics of primary cell transformation in our NCC transformation model via a limiting dilution in vivo study. Previously published reports in melanoma cells determined stemness using a rate-limiting dilution series down to 1 initiating cell (Quintana et al., 2008). Using our modified primary cells, we tested a 2-fold dilution series from $6 \times 10^{6}$ to $0.75 \times 10^{6}$ cells per mouse to determine if restricting cell number would exacerbate tumor initiation potential and identify if any stem-like qualities were present in the different groups.

Tumor kinetics at tumor onset $\left(300 \mathrm{~mm}^{3}\right)$ and tumor endpoint $\left(1500 \mathrm{~mm}^{3}\right)$ were analyzed. Kaplan-Meier curve and statistical analysis indicated significantly reduced latency in the groups injected with $1.5 \times 10^{6}$ and $.75 \times 10^{6}$ cells per mouse relative to WT1 (Figure 3-1D and E). WT1 cells resulted in 71\% overall penetrance (WT1, $n=10 / 14$ ) while HET cells resulted in 100\% penetrance (HET, $n=14 / 14$ ), and time until tumor initiation $\left(300 \mathrm{~mm}^{3}\right)$ was a median of 90.5 days in WT1 and 56 days in HET across all mice in the study (Figure 3-2A). Both WT1 and HET end-point tumors were highly proliferative, as measured by immunohistochemistry (IHC) of Ki67 and phospho- 
Figure 3-1. Heterozygous Ardila deletion increases transformation kinetics of Mycn overexpression in primary trunk NCCs.

(A) Arid $1 a^{\text {flox/wild-type }}$ and Arid $1 a^{\text {flox/flox }}$ NCCs were generated from Aridla floxed mice. Next, adeno-gfp or adeno-cre virus was used to transduce floxed cells in vitro, Mycn was overexpressed (Mycn OE) using retrovirus, and cells were flank injected into mice. (B-E) Matched pairs of Aridla $a^{\mathrm{WT} 1}$ or Aridla ${ }^{\mathrm{HET}} \mathrm{NCCs}+$ Mycn OE were injected in a 2x limiting dilution series into SCID mice. Kaplan-Meier plot of end point tumor growth kinetics $\left(1500 \mathrm{~mm}^{3}\right)$ in WT1vs HET using NCCs injected at the following numbers in each mouse

(B) $6 \mathrm{e} 6(\mathrm{n}=3$ per group), ns. $p=0.343$,

(C) $3 \mathrm{e} 6,(\mathrm{n}=4$ per group), ns. $p=0.241$

(D) $1.5 \mathrm{e} 6,\left(\mathrm{n}=4\right.$ per group), ${ }^{*} p=0.027$ and

(E) $0.75 \mathrm{e} 6(\mathrm{n}=3$ per group), $* p=0.025$

(F-I) Matched pairs of Aridla $a^{\mathrm{WT} 2}$ or Arid1 $a^{\mathrm{KO}} \mathrm{NCCs}+$ Mycn OE were injected in a 2x limiting dilution series into SCID mice. Kaplan-Meier plot of end point tumor growth kinetics $\left(1500 \mathrm{~mm}^{3}\right)$ in WT2 vs KO using NCCs injected at the following numbers in each mouse

(F) $6 \mathrm{e} 6(\mathrm{n}=3$ per group), ns. $p=0.110$,

(G) $3 \mathrm{e} 6,(\mathrm{n}=4$ per group) ns. $p=0.268$

(H) $1.5 \mathrm{e} 6,(\mathrm{n}=4$ per group) $\mathrm{ns} . p=0.248$ and

(I) $0.75 \mathrm{e} 6$ ( $\mathrm{n}=3$ per group), ns. $p=0.110$.

$p$-values were calculated using the Log-rank (Mantel-Cox) test; $(* p \leq 0.05, * * p \leq 0.01)$. 


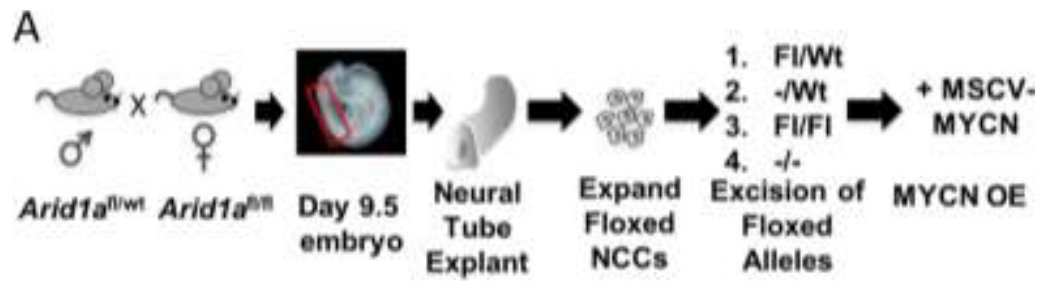

B

C

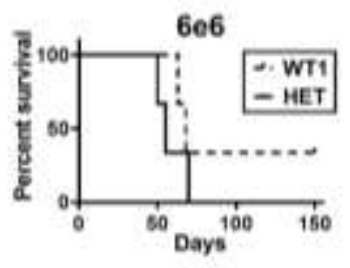

D
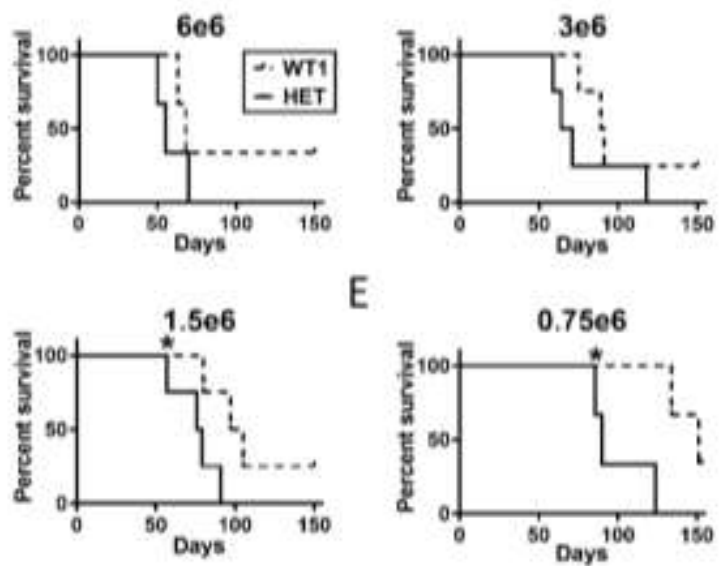

E

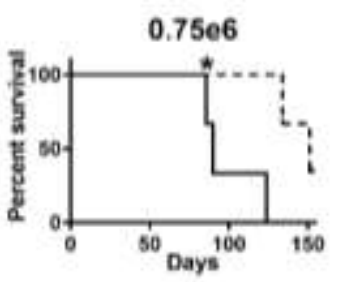

F

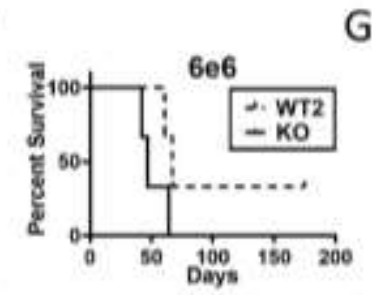

G

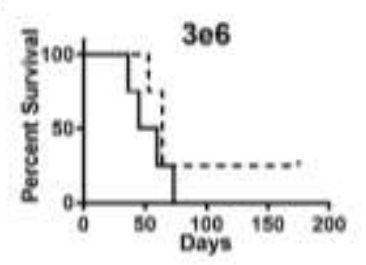

H
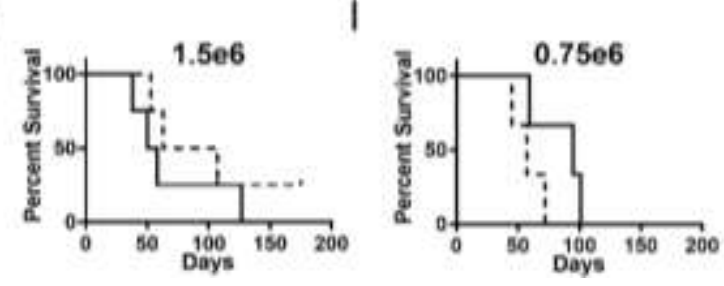


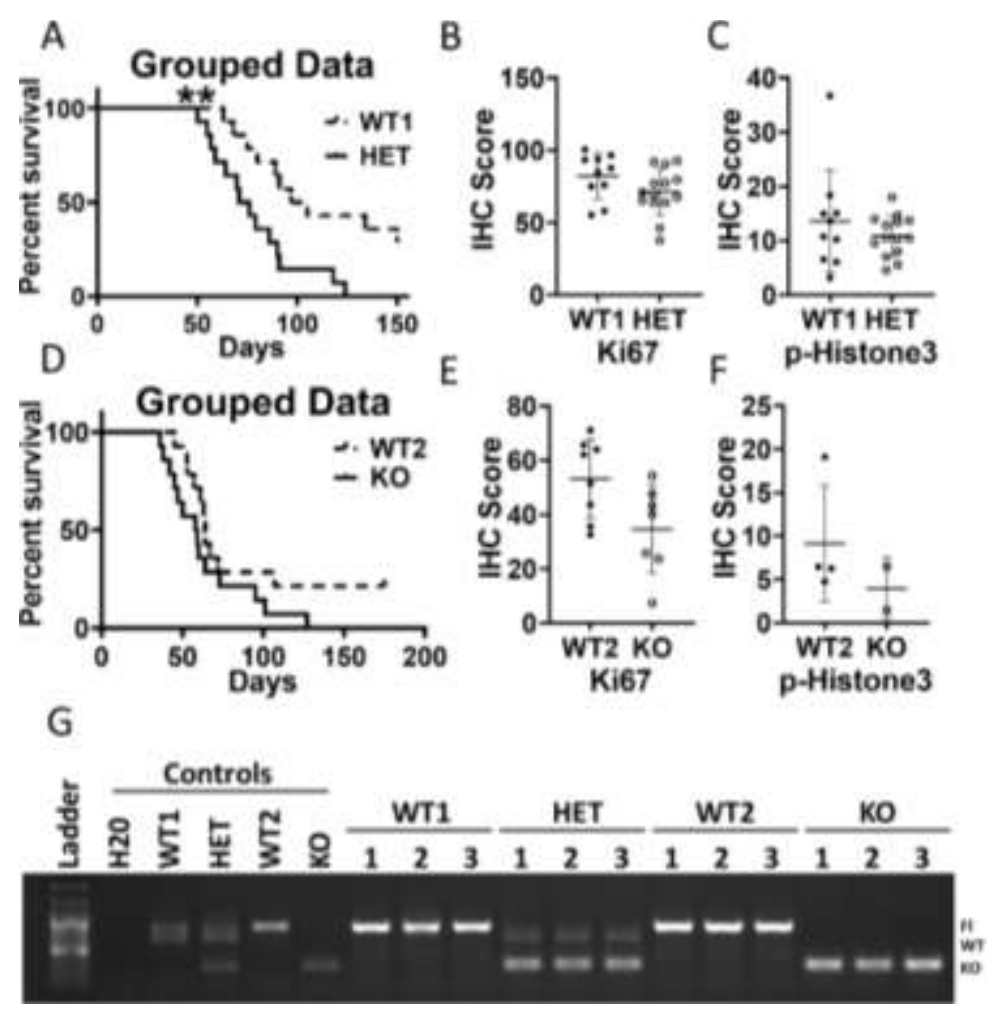

Figure 3-2. Grouped data recapitulates trends in Kaplan-Meier limiting dilution graphs.

(A) Grouped data from Figure 3-1 limiting dilution series for WT1 and HET. KaplanMeier plot of end point tumor growth kinetics $\left(1500 \mathrm{~mm}^{3}\right)$ in HET vs WT1; total mice injected per group $(\mathrm{n}=14) * * p=0.004$.

(B and C) Quantification of WT1 vs HET tumors at endpoint following IHC for proliferative markers Ki67 (WT1 n = 9, HET $=14) * \mathrm{P}=0.039$ and p-HistoneH3 (WT1 n $=9$, HET $=14)$ ns. $p=0.336$. Samples were analyzed by color deconvolution analysis.

Points represent staining and quantification from individual tumors.

(D) Grouped data from Figure 3-1 limiting dilution series for WT2 and KO. KaplanMeier plot of end point tumor growth kinetics $\left(1500 \mathrm{~mm}^{3}\right)$ in WT2 vs KO; total mice injected per group $(\mathrm{n}=14)$ ns. $p=0.093$.

(E and F), Quantification of WT2 vs KO tumors at endpoint following IHC for proliferative markers Ki67 (WT2 $\mathrm{n}=8, \mathrm{KO} \mathrm{n}=7$ ) ns. $p=0.054$ and $\mathrm{p}$-HistoneH3 (WT2 $\mathrm{n}=4, \mathrm{KO} \mathrm{n}=2)$ ns. $p=0.800$. Samples were analyzed by color deconvolution analysis. Points represent staining and quantification from individual tumors. (G), DNA genotyping analysis of Aridla in representative tumor derived cell lines derived from endpoint tumors. Primary cells were used as controls.

Grouped tumor study $p$-values were calculated using the Log-rank (Mantel-Cox) test; Ki67 and $\mathrm{pH} 3$ analysis generated P-values using Mann Whitney test $\left({ }^{*} p \leq 0.05,{ }^{* *} p \leq\right.$ $0.005)$. IHC Score $=1.0 *(\%$ Weak $)+2.0 *(\%$ Medium $)+3.0 *(\%$ Strong $)$. IHC $=$ immunohistochemistry, $\mathrm{pH} 3=\mathrm{p}$-HistoneH3. $\mathrm{Fl}=$ intact Aridla floxed allele at $845 \mathrm{bp}$. WT $=$ intact Aridla wild-type allele at $669 \mathrm{bp} . \mathrm{KO}=$ excised Aridla allele $(\mathrm{HET})$ or alleles (KO) at $298 \mathrm{bp}$. 
histone3 (pH3) analysis (Figure 3-2 B and $\mathbf{C}$ ). To further investigate the effects of homozygous loss of Aridla, we performed the same experiment in a matched pair of NCCs to compare WT2 versus KO tumor growth characteristics. We found no significant differences in tumor latency in KO compared to WT2 analyzed at tumor onset $\left(300 \mathrm{~mm}^{3}\right)$ and tumor endpoint $\left(1500 \mathrm{~mm}^{3}\right)$ in any of the four cell dilutions tested (Figure 3-1E and H). However, KO tumors did have an increased penetrance of $100 \%(\mathrm{KO}, \mathrm{n}=14 / 14)$ compared to the WT2 group's overall penetrance of $79 \%$ (WT2, n=11/14). Time until tumor initiation across all WT2 samples was a median of 55.5 days and 47 days in KO (Figure 3-2D). Furthermore, tumors were highly proliferative, which was measured by IHC against Ki67 and pH3 molecular markers (Figure 3-2E and F). To confirm that the initial Aridla deletions were maintained throughout tumorigenesis, tumor derived cell lines (TDCLs) from representative samples were subjected to genotyping analysis, which confirmed the expected Aridla status in the samples analyzed (Figure 3-2G). These data suggest a dose dependent requirement for Aridla during MYCN-driven NCC transformation.

To validate the findings of the limiting dilution experiment, NCCs were generated, and genetically modified in matched groups as described above and mouse numbers were expanded per group. These results further support the findings of the first study. Again, HET NCCs transformed more readily than WT1 as indicated by KaplanMeier analysis (Figure 3-3A). Furthermore, no tumor initiation advantage was observed in $\mathrm{KO}$ tumors compared to controls (Figure 3-3C). Tumor tissue was analyzed by genomic PCR for Aridla genotype, which confirmed that tumor genotypes were consistent with the original genetic manipulations in parental NCCs (Figure 3-3B and D). Furthermore, the expected Aridla and Mycn expression levels were confirmed by western analysis of final tumors (Figure 3-4A to $\mathbf{C}$ ). Results were consistent with the expected outcomes based on genetic manipulations. Taken together, these studies indicate that Aridla heterozygous deletion is a driver mutation that cooperates with $M Y C N$ in the context of $M Y C N$-driven NBL, which is consistent with the expected phenotype of a 1p36 haploinsufficient TSG.

\section{Gene expression and pathological and molecular characterization of in vivo Arid1a model}

Four tumors per group ( 16 total) were subjected to deep sequencing analysis (RNA-seq). Unsupervised hierarchical clustering analysis of RNA-seq expression levels revealed that tumor samples grouped based on Aridla status (Figure 3-5A), specifically, WT1 and WT2 grouped together, while HET and KO tumors grouped together, and were divided into 10 distinct groups. Our goal is to identify set of Aridla dependent upregulated genes that may indicate a cellular pathway or mechanism involved in NCC transformation; therefore, we chose to focus on genes that were determined to be upregulated in both HET and KO, but not WT1 or WT2 (light blue = group 1). This group consisted of 800 genes. Many genes in this group have been identified previously as genes consistent with de-reugulated expression profiles seen in NBL. In this study, 
A
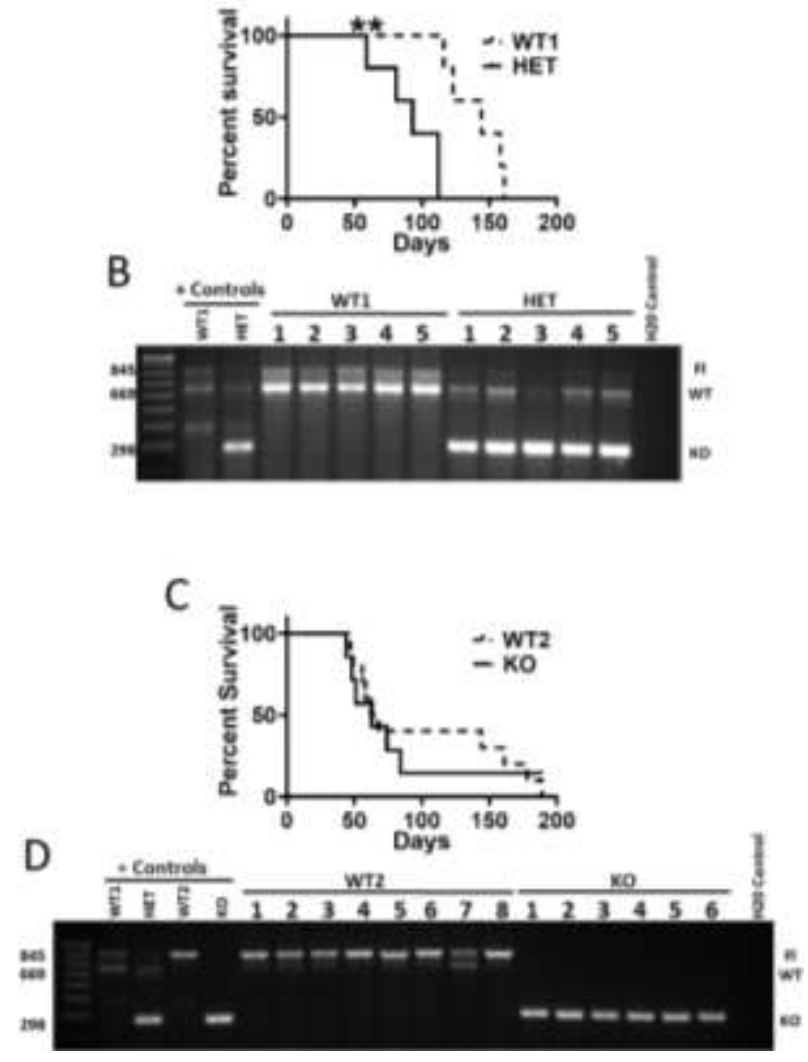

Figure 3-3. Heterozygous Arid1a synergizes with MYCN-driven NBL. (A and C) Aridl $a^{\text {flox/wild-type }}$ and Aridl $a^{\text {flox/flox }}$ NCCs were generated from Aridla floxed mice. Next, adeno-gfp or adeno-cre virus was used to transduce floxed cells ex vitro, Mycn was overexpressed (Mycn $\mathrm{OE})$ using retrovirus, and cells were flank injected into mice.

(A) Matched pairs of Aridla $a^{\mathrm{WT} 1}$ or Aridla ${ }^{\mathrm{HET}} \mathrm{NCCs}+$ Mycn OE were injected at $1.5 \mathrm{e} 6$ cells per mouse into nude mice. Kaplan-Meier plot of end point tumor growth kinetics $\left(1500 \mathrm{~mm}^{3}\right)$ in WT1 vs HET groups; ( $=5$ per group), ${ }^{*} p=0.003$,

(B) DNA genotyping analysis of Arid1a in representative tumor derived cell lines derived from endpoint tumors. Primary cells were used as controls.

(C) Matched pairs of Arid1 $a^{\mathrm{WT} 2}$ or Arid1 $a^{\mathrm{KO}} \mathrm{NCCs}+$ Mycn OE were injected at $1.5 \mathrm{e} 6$ cells per mouse into nude mice. Kaplan-Meier plot of end point tumor growth kinetics $\left(1500 \mathrm{~mm}^{3}\right)$ in WT2 vs KO groups; (WT2 $\mathrm{n}=10$, KO $\mathrm{n}=7$ ), ns. $p=0.954$,

(D) DNA genotyping analysis of Aridla in endpoint tumors. Primary cells were used as controls.

$p$-values were calculated using the Log-rank (Mantel-Cox) test; $(* p \leq 0.05, * * p \leq 0.005)$. $\mathrm{Fl}=$ intact Aridla floxed allele at $845 \mathrm{bp} . \mathrm{WT}=$ intact Aridla wild-type allele at $669 \mathrm{bp}$. $\mathrm{KO}=$ excised Aridla allele (HET) or alleles (KO) at $298 \mathrm{bp}$. 

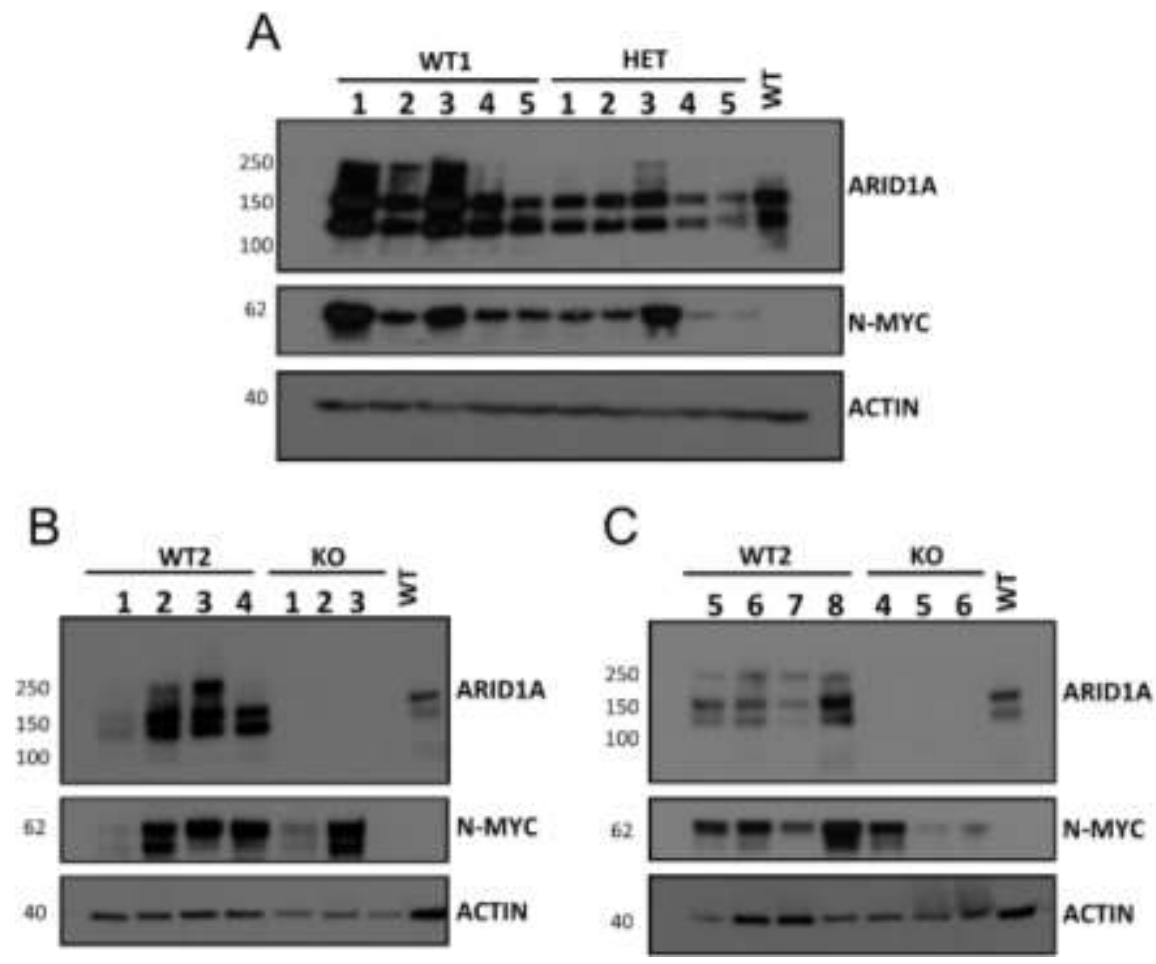

Figure 3-4. Loss of Arid1a expression is consistent with Aridla genotype in end point tumors.

(A through C) Western analysis of end point tumors from Figure 3-3.

(A) ARID1A and N-MYC expression relative to actin loading control in WT1 and HET tumors.

(B and C) ARID1A and N-MYC expression relative to actin loading control in WT2 and KO tumors. 

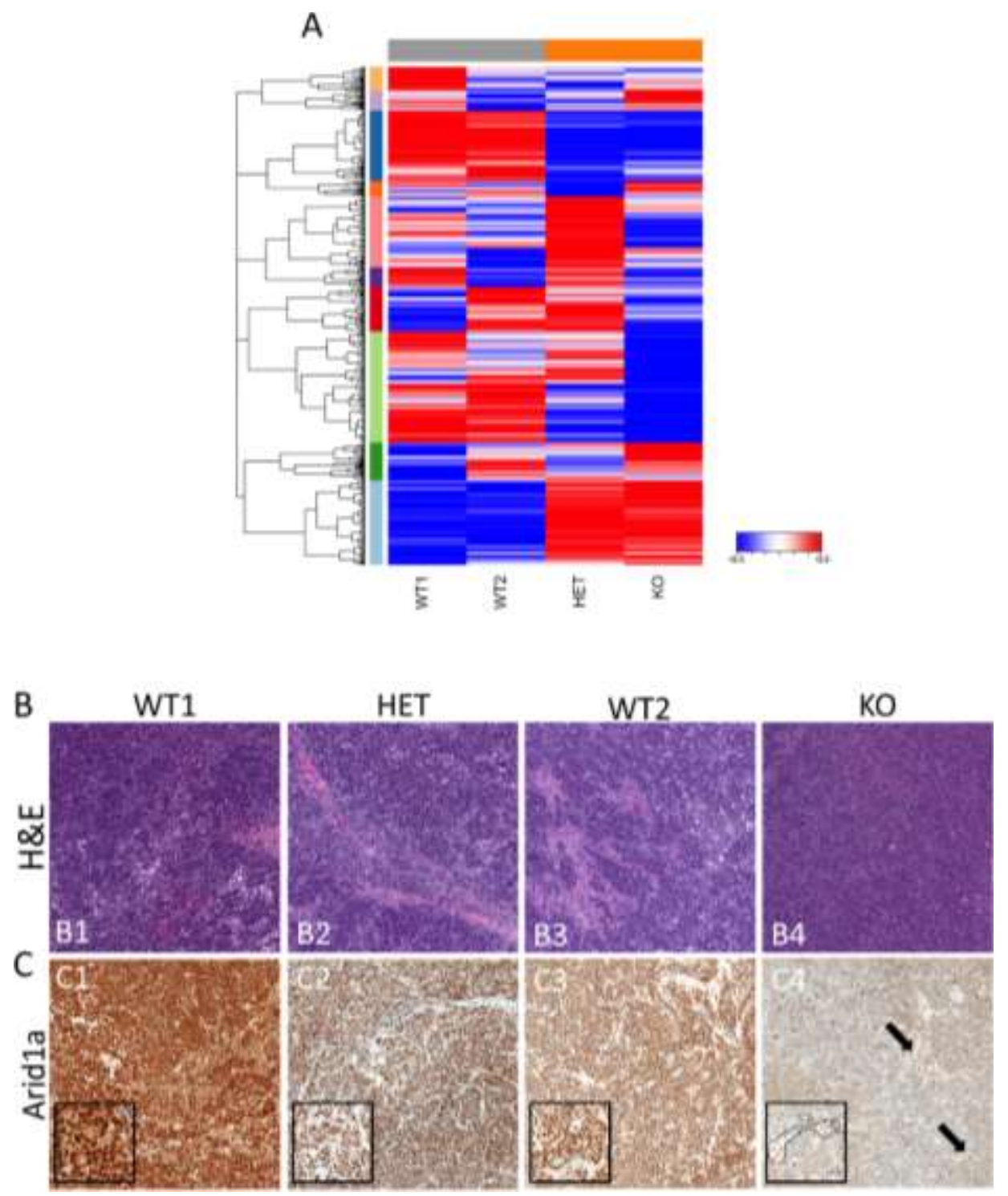

Figure 3-5. RNA-sequencing and histological characterization distinguishes HET and $\mathrm{KO}$ tumors from controls.

(A) Unsupervised hierarchical clustering heatmap of mean tumor samples reveals differentially expressed genes generated from mRNA-sequencing analysis. Samples are grouped into $\mathrm{k}=2$ clusters (gray and orange, top). Representative genes are grouped according to the row dendrogram height of clustered genes (various colors, left). (B1 through 4) Representative H\&E images of WT1, HET, WT2 and KO tumors, respectively. (C1 through 4) Representative IHC protein analysis images of Aridla staining in WT1, HET, WT2 and KO tumors, respectively. $\mathrm{IHC}=$ immunohistochemistry, $\mathrm{H} \& \mathrm{E}=$ Haemotoxylin and Eosin. 
Twist1, Sox9, Foxc1, Zeb1, CD96, Snai2, Snai1, Shh, Pdgfra, Eya1/2, Rara, Vnn1, Cdk6, Six1, Trim7, Runx 1, Wnt9, Ptch2, Hox9/10, Tert, Ascl2, Hoxa1 1, Shb, NeuroD1, and Trim 71 gene expression were upregulated in both Aridla-depleted grouped samples (8 total tumors). Downregulated genes consisted of 670 genes downregulated in both HET and KO, but not in WT1 or WT2. These genes were analyzed by STRING, a bioinformatics tool that determines the likelihood of connections in biological pathways based on a gene list. This analysis determined that the down-regulated genes were predicted to be biologically connected (PPI enrichment $\mathrm{p}$-value $=1 \times 10^{-16}$ ). The top GO Biological processes affected were nervous system development, neurogenesis, and the generation of neurons (Table 3-1).

Pathological review from board-certified veterinary pathologist Dr. Heather Tillman indicated that tumor morphologies were consistent with $M Y C N$-driven NBL. The tumors were locally invasive, but metastatic spread was not observed in full-body hematoxylin and eosin (H\&E) analysis from representative samples, nor in lymph, lung, or liver from samples collected at end-point analysis. Functional loss of Aridla in murine neural crest progenitor cells resulted in an undifferentiated NBL histology. WT tumors show limited neuroblastomic differentiation and a high mitosis-karyorrhexis index that is consistent with the histology of human tumors that have amplified MYCN (WT1, WT2) (Figure 3-5B1 and B3). Furthermore, WT1 and WT2 tumors were consistent with Schwannian-poor NBL (Shimada classification), with regions of undifferentiated, poorly differentiated, or differentiating cellular morphology highlighted in the H\&E sections (Figure 3-5B1 and B3). Reduction (HET) or loss (KO) of Aridla further synergizes with Mycn OE resulting in an earlier arrest in differentiation in transformed neural crest cells. Subpopulations within HET and KO allografts that have genetic and morphologic changes that are reminiscent of embryonic ectoderm and mesenchymal cells that are also able to give rise to terminally differentiated cell types, such as sensory neurons and glia, within the bulky tumor as detected by IHC (Figure 3-5B2 and B4). HET and KO groups presented with greater populations of poorly differentiated or undifferentiated morphologies compared to controls (Figure 3-5B2 and B4). All tumor samples were analyzed by IHC for Aridla expression. Genetic targeting of Aridla resulted in the reduction of detection of protein expression by IHC. WT1 have nuclear localization of Aridla while HET tumors have a reduction and partial loss of nuclear localization of Arid1 a (Figure 3-5C1 and C2). Arid1 a expression is drastically reduced in the knockout tumors by IHC relative WT2 control (Figure 3-5C4 and C2, respectively). Arid1a immunoreactivity remains localized to the nucleus of mouse host tissues when observed in KO tissue (Figure 3-5C4, arrows, insert, dotted lines). In summary, these findings are consistent with a phenotype characterized by deregulated differentiation in Aridla depleted tumors.

\section{Aridla loss causes a differentiation block in vivo and in vitro}

NBL arises from a transient population of migratory trunk NCCs that specify into Tuj1+ neurons, or GFAP+ glia of the SNS. 
Table 3-1. GOBiological processes affected by Arid1a-dependent down-regulated genes.

\begin{tabular}{llcc}
\hline GO-term & \multicolumn{1}{c}{ Description } & Count in gene set & FDR \\
\hline GO:0007399 & Nervous system development & 156 of 2181 & $2.40 \times 10^{-22}$ \\
GO:0022008 & Neurogenesis & 127 of 1650 & $4.11 \times 10^{-20}$ \\
GO:0048699 & Generation of neurons & 117 of 1538 & $5.45 \times 10^{-18}$ \\
GO:0048731 & System development & 211 of 4072 & $1.02 \times 10^{-15}$ \\
GO:0007275 & Multicellular organism development & 225 of 4603 & $2.55 \times 10^{-14}$ \\
\hline
\end{tabular}

GO STRING analysis of top biological processes affected by concordant HET and KO down-regulated genes. FDR $=$ False discovery rate. 
One trait of HR NBL is poorly differentiated tumors and a block in SNS development that are thought to contribute to NBL oncogenesis (Jiang, 2011). We used IHC to analyze markers of sympathoadrenal development to characterize the differentiation status of our model. We analyzed end stage tumors by IHC using molecular markers to measure neuronal and glial commitment. Tuj1 is an early, post-mitotic neuronal marker, present during NCC differentiation into SNS neurons. Both the HET and KO tumor samples demonstrated significantly reduced Tuj1 expression compared to control tumor samples, suggesting blocked neuronal differentiation (Figure 3-6A and $\mathbf{C}$ ). A decrease in neuronal differentiation suggested that Het and KO tumors would demonstrate one of two traits, 1 . an increase of glial cells suggestive of re-distribution of lineage commitment or 2. a decrease of glial cells symptomatic of a block in SNS commitment to the glial lineage. Analysis of GFAP positivity in tumors indicated no significant difference of GFAP expression in HET compared to WT1 tumors (Figure 3-6B), however, KO samples showed significantly reduced in GFAP expression relative to WT2 (Figure 3-6D). These results suggest that Aridla HET deletions inhibit neuronal differentiation, and $\mathrm{KO}$ deletions result in a more severe block, perturbing both neuronal and glial differentiation. Due to the Aridla dependent differences in tumor differentiation, we wanted to further test the effects of Aridla dose in vitro, to determine if Aridla loss alone could contribute to a block in SNS development.

To evaluate if Aridla loss affected differentiation in untransformed NCCs, we chose to work with the parental primary NCCs that harbored Aridla deletions, and wildtype $M y c n$. These cells were subjected to neural differentiation media and neuronal differentiation properties were tested in vitro. Confocal immunoflurorescent (IF) analysis was used to evaluate neuronal differentiation. Images for quantification were captured at random. As indicated in Figure 3-6E and G, Neuronal projections were evident in WT1 and WT2 fields of view but were reduced in an Aridla dose-dependent pattern. Consistent with the tumor data, we found that both Aridla ${ }^{+/-}$cells and Arid $1 a^{-/-}$cells exhibited a reduced number of neurons compared to controls (Figure 3-6F and $\mathbf{H}$ ). This finding was also demonstrated in WT cells transfected with different gRNAs to target Arid 1 a using CRISPR/CAS9 technology. Consistent with the floxed NCC data, Arid1a depleted cells recapitulate the reduction in NCC neuronal differentiation (Figure 3-7B). In summary, analysis of SNS differentiation distinguished WT1 and WT2 groups from less differentiated HET and $\mathrm{KO}$ groups. This data indicates that the progression of $\mathrm{NCC}$ differentiation was altered when Aridla expression was reduced or absent, with and without the presence of Mycn OE.

\section{Arid1a loss up-regulates genes associated with a mesenchymal cell identity}

Aridla HET deletions in vivo and in vitro resulted in a block in neuronal commitment, while Aridla KO blocked neuronal and glial differentiation. Differentiation programs involve a vast number of genes, and because ARID1A is an epigenetic regulator, Aridla loss may alter the expression of many genes that regulate development. 
Figure 3-6. Arid1a loss blocks differentiation in vivo and in vitro.

(A and B) Quantification of WT1 vs HET tumors at endpoint following IHC for differentiation markers Tuj1 (WT1 $\mathrm{n}=9$, HET $\mathrm{n}=13) * \mathrm{P}=0.0208$ and GFAP (WT1 $\mathrm{n}=$ 9 , HET $\mathrm{n}=13$ ) ns. $p=0.601$ as analyzed by color deconvolution analysis. Points represent staining and quantification from individual tumors.

(C and D) Quantification of WT2 vs KO tumors at endpoint following IHC for differentiation markers Tuj1(WT2 $\mathrm{n}=8, \mathrm{KO} \mathrm{n}=6) * * p=0.008$ and GFAP (WT2 $\mathrm{n}=8$, $\mathrm{KO} \mathrm{n}=5) \mathrm{ns} .{ }^{*} p=0.030$ as analyzed by color deconvolution analysis. Points represent staining and quantification from individual tumors. (E through H) Primary NCCs were cultured in differentiation media for one week, costained for ARID1A and MAP2, then cell morphology was analyzed using immunofluorescence (IF).

(E) Representative staining of NCC neurons in WT1 and HET populations. (F) Quantification of neurons counted in each field captured across $n=5$ experiments. Images analyzed were captured at random. ${ }^{*} p=0.0353$.

(G) Representative staining of NCC neurons in WT2 and KO populations. (H) Quantification of neurons counted in each image captured across $n=4$ experiments. Images analyzed were captured at random. $* * p=0.0008$.

(A, B, C and D) Tuj1 and GFAP analysis $p$-values were generated using Mann Whitney test. (F and $\mathrm{H}$ ) Neuron quantification $p$ values were calculated using an unpaired test with Welch's correction; $(* p \leq 0.05, * * p \leq 0.01, * * * p \leq 0.0005)$. ARID1A $=$ red, MAP2 $=$ green, $\mathrm{DAPI}=$ blue. 

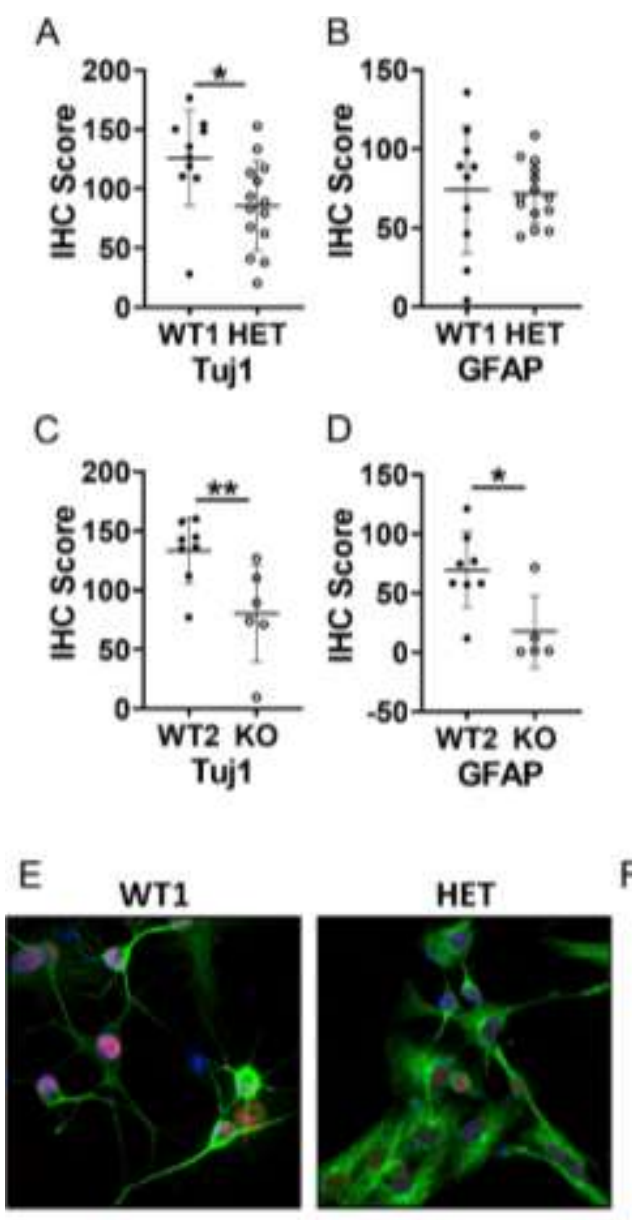

F
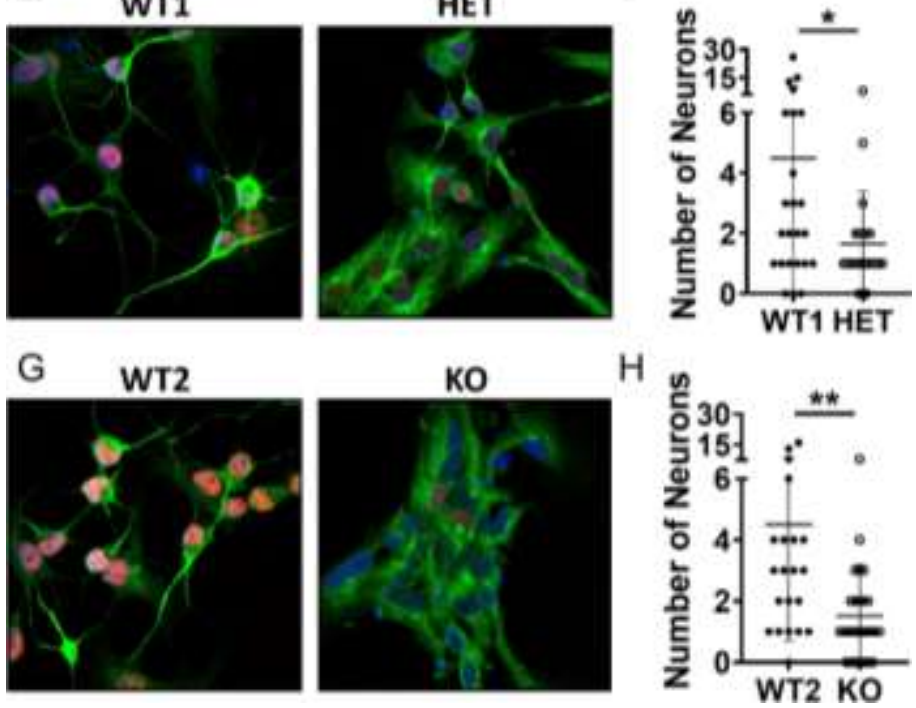

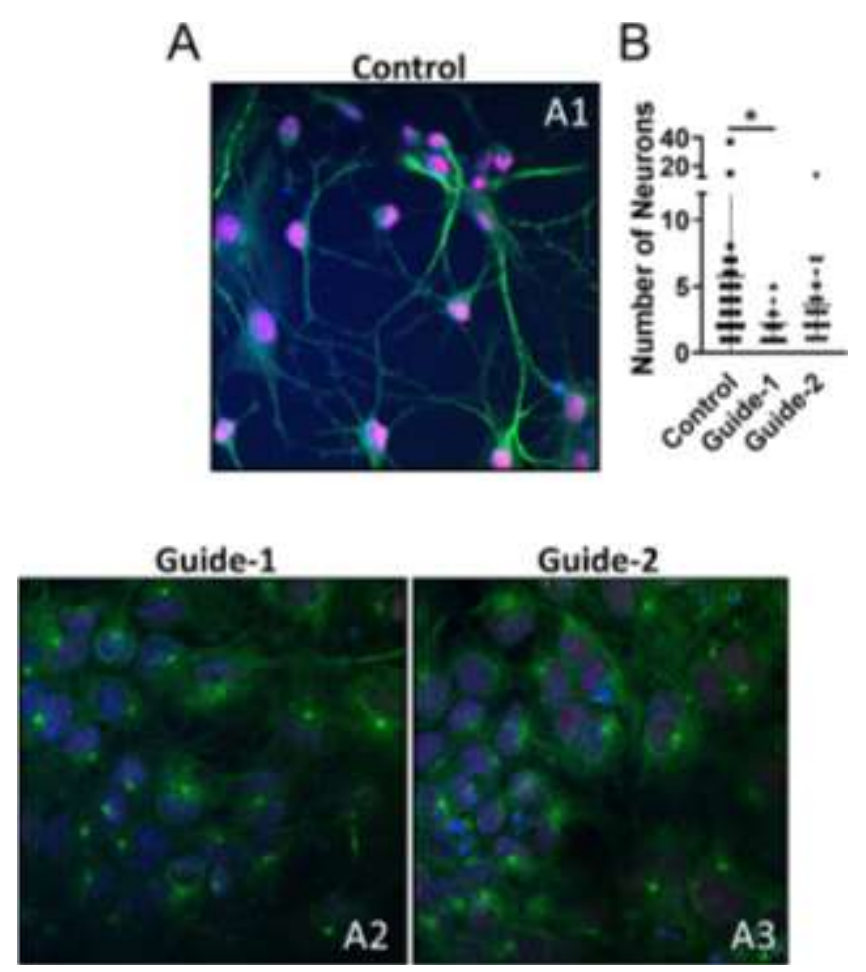

Figure 3-7. CRISPR-mediated deletion of Arid1a impaired neuronal differentiation.

(A) Wild type primary NCCs were electroporated with CRISPR guides to Aridla (Guide1 and Guide-2), or a non-targeting guide (Control). Cells were placed in differentiation media for one week, co-stained for ARID1A and MAP2, then cell morphology was analyzed using IF.

(A1 through A3). Representative staining of NCC neurons in control and Aridla modified populations.

(B) Quantification of neurons counted across $n=4$ experiments. Images analyzed were captured at random. Control vs. Guide- $1 * p=0.038$. Control vs. Guide-2 ns. $p=0.239$. $p$ values were calculated using an unpaired t test with Welch's correction; $(* p \leq 0.05)$. Arid1 $\mathrm{a}=$ red, Map2 $=$ green, DAPI $=$ blue. 
Therefore, we further analyzed our RNA-seq tumor comparisons to identify signaling nodes that are up regulated in response to Aridla depletion. The gene list included genes that are concordantly up-regulated in Aridla HET and KO tumors compared to WT1 and WT2 control tumors (Figure 3-5A light blue - group1). This gene list was analyzed using STRING protein analysis to identify possible functional protein associations. Outputs were constrained to high-confidence data from experimental, textmining, and database sources. The final list included 753 unique upregulated nodes and a PPI enrichment pvalue of 1e-16, indicating a significant amount of interactions that signifies connection among groups of biologically connected genes (Figure 3-8A). Multiple signaling hubs included genes involved in migration, EMT, and development (Figure 3-8A). EMT has been shown in multiple adult cancer models to be regulated by Aridla depletion (Wang, Gut, 2019, Sun, 2016, Cancer Cell). Furthermore, we identified a node comprised of multiple EMT-regulating genes that were connected to Twist1 (Figure 3-9A) These genes included Twist1, Sox9, Snai1/2, Zeb1, and CD44 in our model. TWIST1 is a NCC master regulator and EMT pioneering factor that is associated with poor patient outcomes in MNA NBL cases (Selmi et al., 2015; Valsesia-Wittmann et al., 2004). Recently, a landmark NBL study established that TWIST1 is necessary to regulate the oncogenic effects of MYCN at enhancers (Zeid et al., 2018). Considering the role of TWIST1 in MYCN-driven NBL, we highlighted the genes connected to the Twist1 node (Figure 39A). Other notable signaling hubs were centered around the genes Adamts, Wnt $9 a, S h h$, Ltbp 1, Six 1, Src, and Hoxa5 (Figure 3-8A).

Taken together, our previous results suggest Aridla loss altered cell fate decisions resulting in blocked NCC differentiation. Therefore, we expected tumors and tumor derived cell lines (TDCLs) to demonstrate phenotypes and gene expression programs of NCC progenitor cells. Migration due to an EMT transition during delamination is a normal process that occurs early during NCC development between days e9.5-11.5. We thought if transformed NCCs were blocked in an undifferentiated state, they would behave similarly to uncommitted and delaminating NCC precursors. Therefore, to test the connection between cell fate and behavior caused by Aridla loss, we measured migration rates of TDCLs. We expected that cells stuck in an uncommitted progenitor state would have higher rates of migration than controls. As we anticipated, Boyden chamber migration assays indicated that HET and KO TDCLs migrated significantly more than controls (Figure 3-9B and C). We also evaluated expression profiles that were altered in tumors and correlated with cell identity. The top hit based on GSEA analysis in KO versus WT2 tumors indicated that KO tumor gene expression values significantly correlated with the Wong Embryonic Stem Cell Core gene signature (Figure 3-9D). However, this signature was not in the top 20 significantly enriched GSEA gene sets in HET tumors versus WT1 tumors. This result indicates that KO tumors share genes that are concordant with the Wong Emrbyonic Stem Cell Core signature. This gene signature is indicative of embryonic stem cell-like transcriptional programs (Wong et al., 2009). Taken together the KO data reinforces the concept that Aridla ablation causes NCCs to be blocked in an immature state during transformation. Our findings in Aridla HET and KO samples imply that NCCs transformed with reduced Aridla levels display a phenotype and gene expression profile consistent with NCC progenitors. 


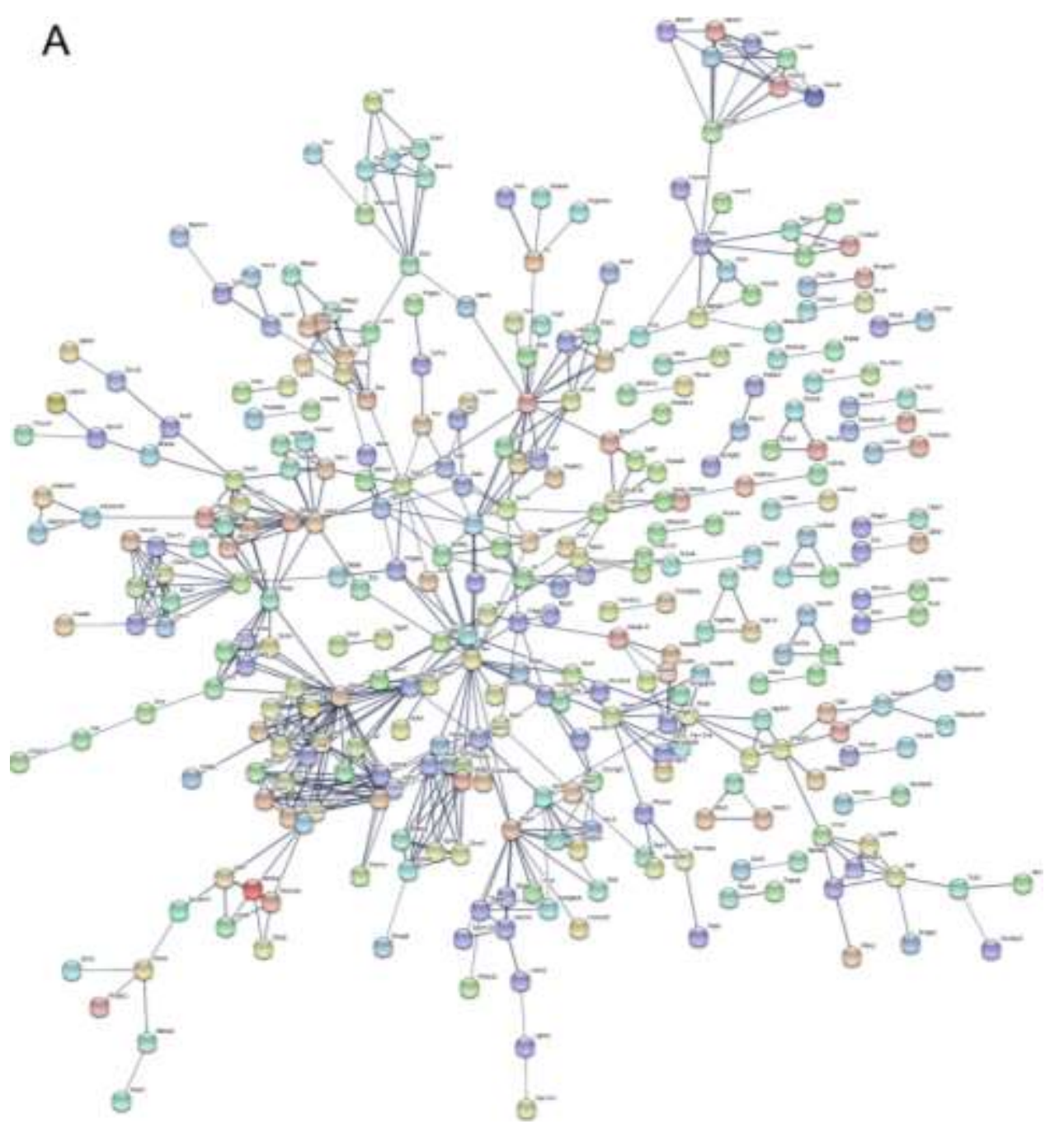

Figure 3-8. Arid1a-dependent up-regulated signaling nodes.

(A) STRING analysis of all genes concordantly up-regulated in HET and KO tumors identified by RNA-seq. Nodes were limited to high confidence textmining, experimental and database mining nodes. 


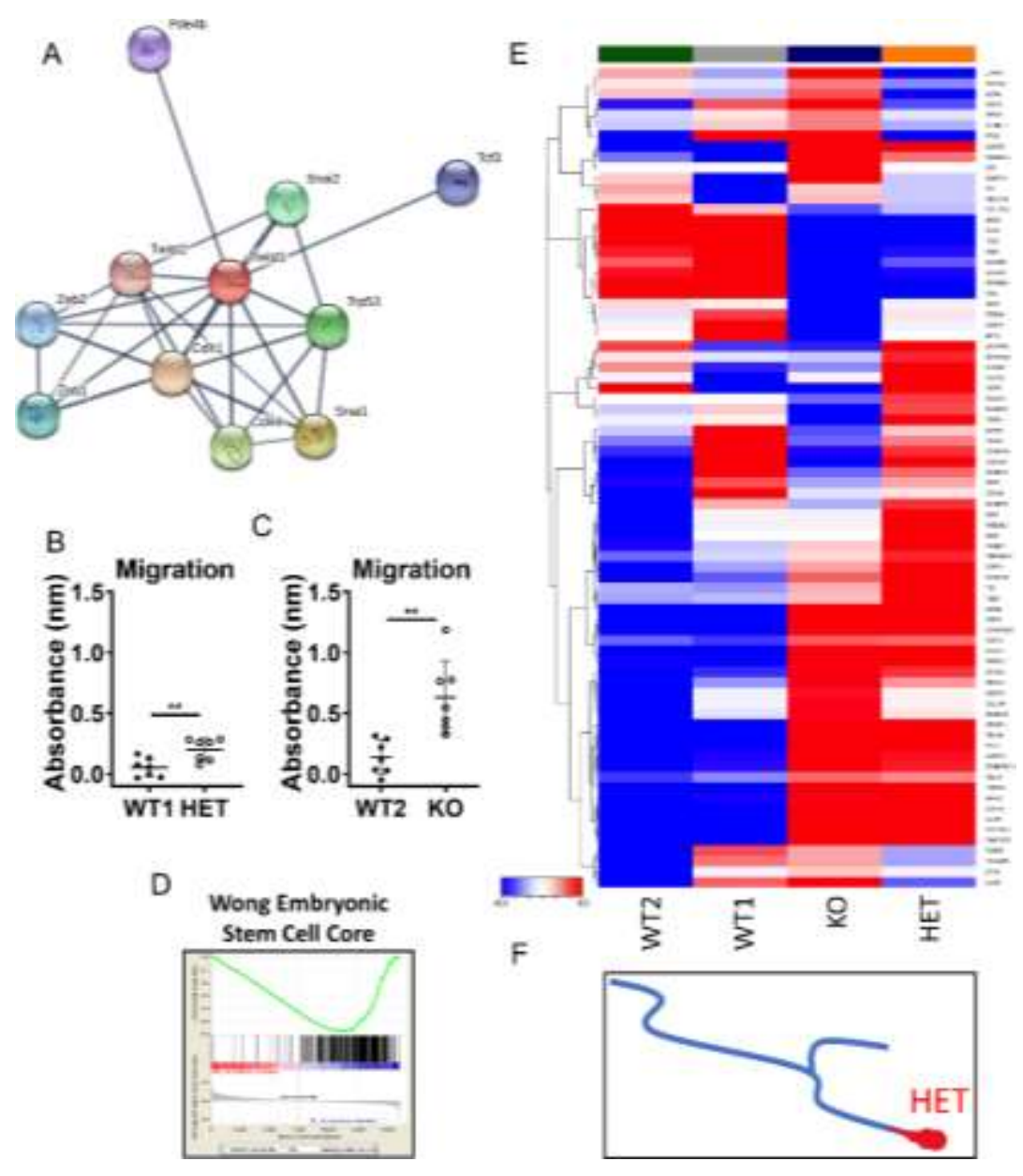

Figure 3-9. Arid1a loss disrupts EMT in vitro and in vivo.

(A) Twist1 signaling node identified by STRING analysis of concordantly up-regulated gene transcripts in HET and KO tumors.

(B and C) Boyden chamber migratory analysis of TDCLs in (B) WT1 and HET $(n=7)$; $* * \mathrm{P}=0.006$; or $(\mathrm{C}) \mathrm{WT} 2$ and $\mathrm{KO}(\mathrm{n}=7) ; * * \mathrm{P}=0.002$.

(D) Gene set enrichment (GSEA) analysis of WT2 versus KO tumors indicated a significant correlation with Wong embryonic stem cell core and KO samples, but not WT2 samples (Wong, 2008, Cell Stem Cell).

(E) Expression heatmap for tumor samples using mesenchymal genes of the cluster 3 gene set described by (Soldatov et al., 2019).

(F) Schematic indicating the mapping of HET samples to mesenchymal genes in cluster 3. (Soldatov et al., 2019).

$(* \mathrm{P} \leq 0.05, * * \mathrm{P} \leq 0.01)$.

Data Source. Soldatov, R., Kaucka, M., Kastriti, M. E., Petersen, J., Chontorotzea, T., Englmaier, L., ... Adameyko, I. (2019). Spatiotemporal structure of cell fate decisions in murine neural crest. Science, 364(6444). https://doi.org/10.1126/science.aas9536

Data Source. Wong, D. J., Liu, H., Ridky, T. W., Cassarino, D., Segal, E., \& Chang, H. Y. (2008). Module Map of Stem Cell Genes Guides Creation of Epithelial Cancer Stem Cells. Cell Stem Cell, 2(4), 333-344. https://doi.org/10.1016/j.stem.2008.02.009 
Our tumor latency data (Figures 3-1 through 3-3) and GSEA analysis suggests a difference between HET and KO tumors. Considering that HET tumors showed a reduced latency relative to WT1 samples and fit the genetic profile of a haploinsufficient 1 p36 TSG, we were interested to determine if HET tumors mapped to a cell fate associated with aggressive cellular behavior. Both HET and KO samples upregulated genes associated with an EMT signature, but the KO versus WT2 GSEA signature Figure 3-9D suggested along with differentiation data from Figure 3-6 that $\mathrm{KO}$ tumor cells have a severely blocked, stem-like NCC identity. In summary our data suggests that HET cells may have a different cell identity than KO cells. A recent paper eloquently mapped NCC gene expression profiles to the physical location and cell fate decision points during mouse development (Soldatov et al., 2019). Through extensive single-cell sequencing studies during trunk NCC differentiation, they developed gene lists that were associated with different cell fates in the developing SNS (Soldatov et al., 2019). This group segmented NCC expression profiles into the following 6 key NCC cell identities: premigratory, delaminating, sensory, glia, autonomic and mesenchymal. Mesenchymal cells are associated with increased cell motility and aggressive behaviors (Dongre \& Weinberg, 2019; Flavahan et al., 2017; Douglas Hanahan \& Weinberg, 2011). We thought this cell state most closely associated with the expression profiles and behavior of our HET samples so far. To test this assumption, we mapped our mean tumor RNAseq data against the cluster 3 list of genes associated with a mesenchymal NCC identity (Soldatov et al., 2019). This revealed that HET tumors had the greatest number of upregulated mesenchymal genes relative to the other three tumor types (Figure 3-9E). Taken together, we interpret these results to suggest that the TSG function of Aridla in trunk NCCs is through the regulation of cell identity.

\section{Arid1a heterozygosity correlates with an immature, mesenchymal cell state}

The mapping of mean tumor gene expression values against the mesenchymal gene list from Soldatov, et al. 2019 highlighted that there were gene expression differences between not only HET and WT1, but also between HET and KO tumors. Following from these findings, we were interested to see how substantially WT, HET, or KO Aridla expression shifted the cell identity in tumor populations toward different NCC identities. Furthermore, we wanted to map our tumors to the established lineage tracing gene lists. We used the comprehensive gene list of NCC master regulator genes generated by Soldatov et al., 2019 as a rubric for comparison. We evaluated the mean expression values in each of our tumor groups against their list to focus on the key regulators of cell identity (Figure 3-10). This analysis determined that WT1 and WT2 gene expression values were most consistent with a glial cell identity. HET tumor gene expression values were most consistent with a mesenchymal cell identity, and KO gene expression values were most consistent with a sensory cell identity.

To look more closely at this result, we also evaluated whether the expression levels of NCC master regulators differed based on Aridla genotype. To do this, we graphed the differentially expressed genes (DEGs) that were identified in at least one tumor group (Figure 3-11A through $\mathbf{C}$ ). 


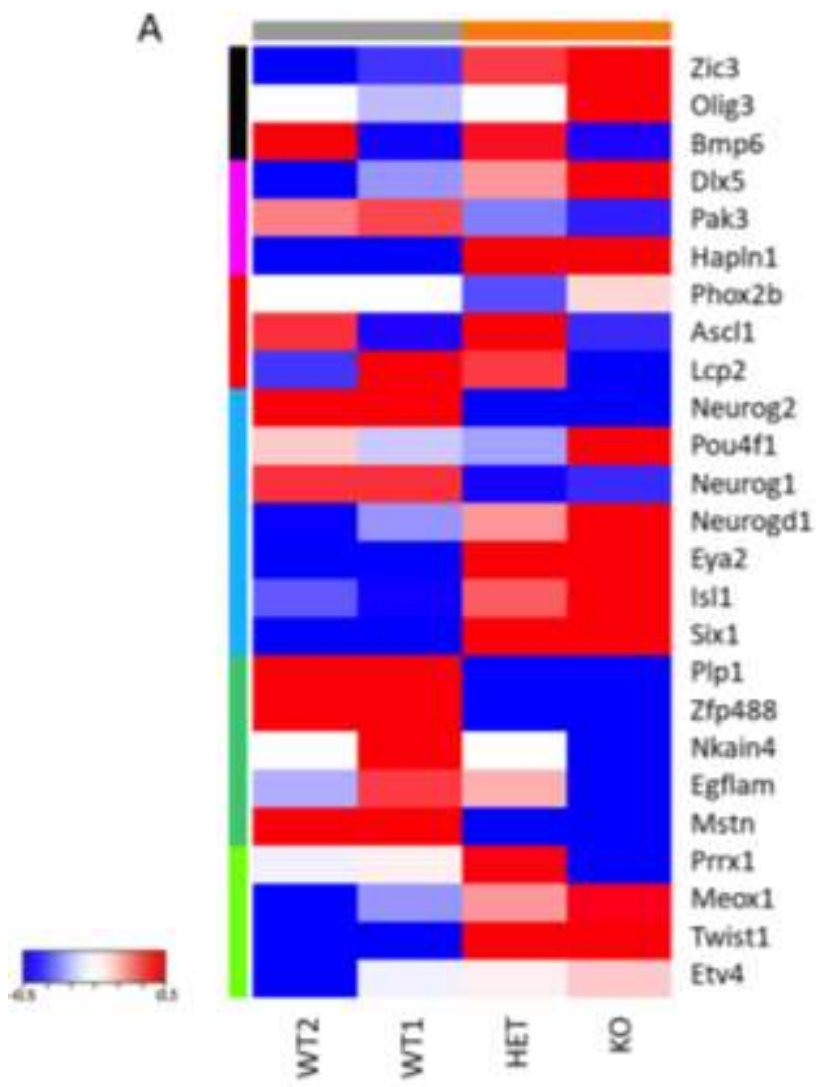

Figure 3-10. Tumor fate mapping reveals differences in HET and KO tumor samples.

(A) Gene expression heatmap for tumor samples using NCC master regulator gene signatures from Soldatov, et al. 2019 revealed differential expression patterns related to cell identity. (B) Schematic mapping of tumors to respective cell identity.

Modified with permission from AAAS. Soldatov, R., Kaucka, M., Kastriti, M. E., Petersen, J., Chontorotzea, T., Englmaier, L., ... Adameyko, I. (2019). Spatiotemporal structure of cell fate decisions in murine neural crest. Science, 364(6444).

https://doi.org/10.1126/science.aas 9536 
Figure 3-11. Differential gene expression analysis reveals alterations of NCC master regulators in tumor samples.

(A through F) Mean tumor values from RNA-sequencing were analyzed for differentially expressed genes (DEGs) between the following tumor samples WT1 vs HET, WT2 vs $\mathrm{KO}$, and HET vs KO ( $\mathrm{n}=4$ in each group). NCC master regulators were plotted to show the Log2 fold-change between each comparison group. Statistical significance was determined using adjusted $p$-values (Benjamini-Hochberg correction).

(A through C) Grouped list showing all master regulators that were altered in our system in at least one comparison group.

(D through E) Summary graphs highlighting NCC master regulators identified as significantly differentially regulated in the comparison listed.

(A) HET gene expression 2 log fold changes were set as the numerator. WT1 gene expression $2 \log$ fold changes were set as the denominator. Positive values represent genes that are up-regulated in HET and down-regulated in WT1. Negative values represent genes that are up-regulated in WT1, but down-regulated in HET.

(B) KO gene expression 2 log fold changes were set as the numerator. WT2 gene expression $2 \log$ fold changes were set as the denominator. Positive values represent genes that are up-regulated in KO and down-regulated in WT2. Negative values represent genes that are up-regulated in WT2, but down-regulated in KO.

(C) HET gene expression $2 \mathrm{log}$ fold changes were set as the numerator. KO gene expression 2 log fold changes were set as the denominator. Positive values represent genes that are up-regulated in HET and down-regulated in KO. Negative values represent genes that are up-regulated in $\mathrm{KO}$, but down-regulated in HET.

(D) Summary of data from (A). Graphical representation of NCC master regulators that were significantly altered in HET and WT1 tumors relative to one another.

(E) Summary of data from (B). Graphical representation of NCC master regulators that were significantly altered in $\mathrm{KO}$ and WT2 tumors relative to one another.

(F) Summary of data from (C). Graphical representation of NCC master regulators that were significantly altered in HET and $\mathrm{KO}$ tumors relative to one another.

Mean tumor values were calculated from 4 individual tumors for each genotype. Error bars were generated using log fold change standard error. Significance was determined using adjusted $p$ values. $(* p \leq 0.05, * * p \leq 0.005, * * * p \leq 0.0005)$. 


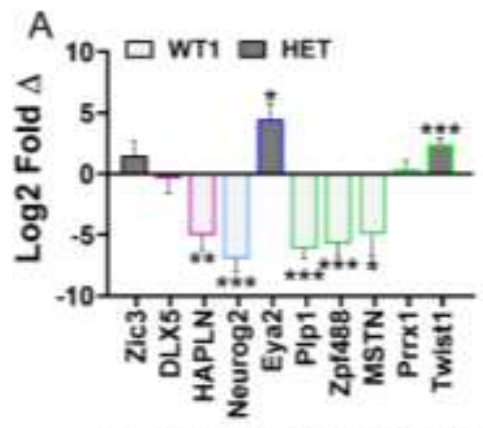

NCC Master Regulators

B

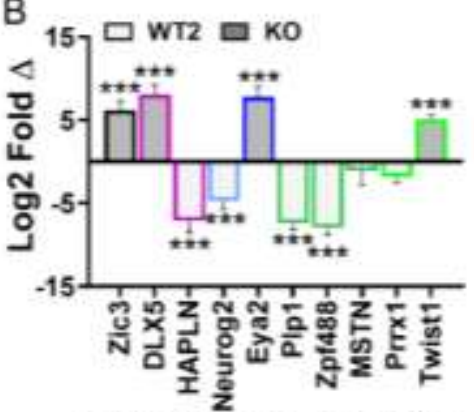

NCC Master Regulators

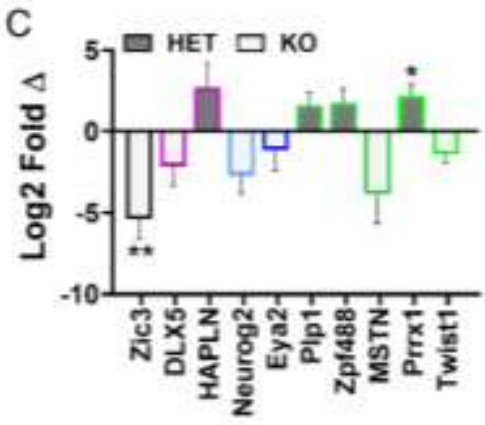

NCC Master Regulators

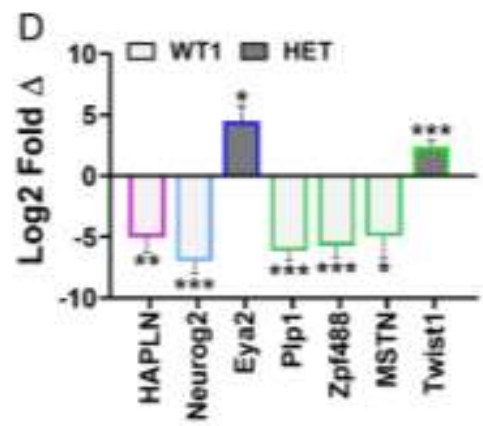

NCC Master Regulators

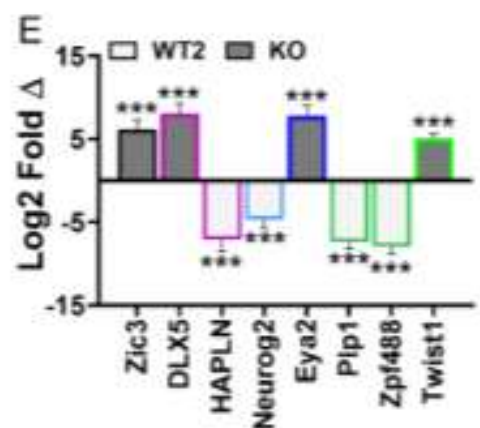

NCC Master Regulators

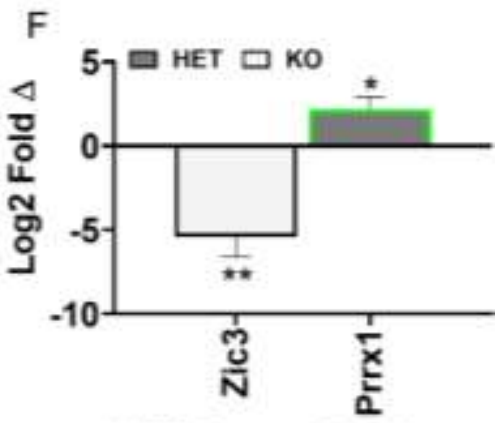

NCC Master Regulators

Premigratory $=$ Delamination $=$ Sensory 1

Eensory2 Glial

Mesenchymal 
Analysis of WT1 and WT2 in comparison to these lists identified two upregulated glial regulators Plp1 and Zfp488. KO tumors had increased Zic3 expression relative to other samples. Both HET and KO had increased Twistl relative to their respective controls. HET tumors demonstrated a modest increase in Prrx 1 relative to all samples, but it was the only gene significantly increased relative to KO samples (Figure 3-11F). This data suggests that WT1 and WT2 have more glial characteristics than HET and KO, with KO displaying high levels of the neural plate boarder specifying factor Zic3, and EMTpioneering factor Twist1, while HET samples had gene expression profiles consisting of increased Twist1 relative to controls, and slightly increased Prrx1 relative to all groups. A summary of this result is depicted in Figure 3-10B.

\section{Arid1a heterozygous tumors gene signature recapitulates human NBL mesenchymal signature}

Through mapping the mean expression values from individual tumor groups, we found that master regulatory genes of mesenchymal NCC cell fate were altered in HET tumors. Following from this finding, we were interested to test whether our mouse model of HR NBL faithfully recapitulates gene expression programs identified in HR NBL patient tumors. One recent NBL study worked to define gene expression programs that could be used to define the heterogeneity found in HR NBL tumors (van Groningen et al., 2017). This work divided the cells comprising HR NBL tumors into two categories, adrenergic (ADRN) and mesenchymal (MES) based on CD133 status and mRNA profile (van Groningen et al., 2017). The adrenergic type (ADRN) was characterized by CD133cells expressing the classic NBL master regulators Phox2A, Phox2B, and DBH, while the mesenchymal (MES) type was comprised of snail, vimentin, and fibronectin mesenchymal markers (van Groningen et al., 2017). MES tumors were stem-like while ADRN were more differentiated (van Groningen et al., 2017). Additionally, the MES signature was correlated to more aggressive tumor behavior in patients (van Groningen et al., 2017). We reasoned that if HET tumors expressed the most mesenchymal genes relative to the remaining groups, as suggested by our data in Figures 3-9 and 3-10, then we would expect our HET samples to display a greater amount of overlap to the human MES signature than other groups. First, we compared our mean tumor expression profiles to the gene list comprised of MES and ADRN associated genes to determine if our mouse model tumor groups overlapped with MES or ADRN signatures identified in patient NBL samples. We found that MES genes were increased in the HET samples, however the KO tumor samples did not demonstrate a strong MES nor ADRN signature (Figure 3-12A). We compared HET versus WT1, WT2, and KO tumor groups by GSEA analysis and determined that the HET mouse model and MES patient tumor signatures overlapped with statistical significance (Figure 3-11B). WT1, WT2, and KO versus HET tumors using GSEA determined that the genes enriched in these groups statistically significantly overlapped with the ADRN patient tumors gene set (Figure 3-11C). This gene set comparison supports the hypothesis that suggests Aridla loss contributes to a SNS developmental block in lineage commitment leading to a cell identity that is associated with aggressive NCC behavior. 


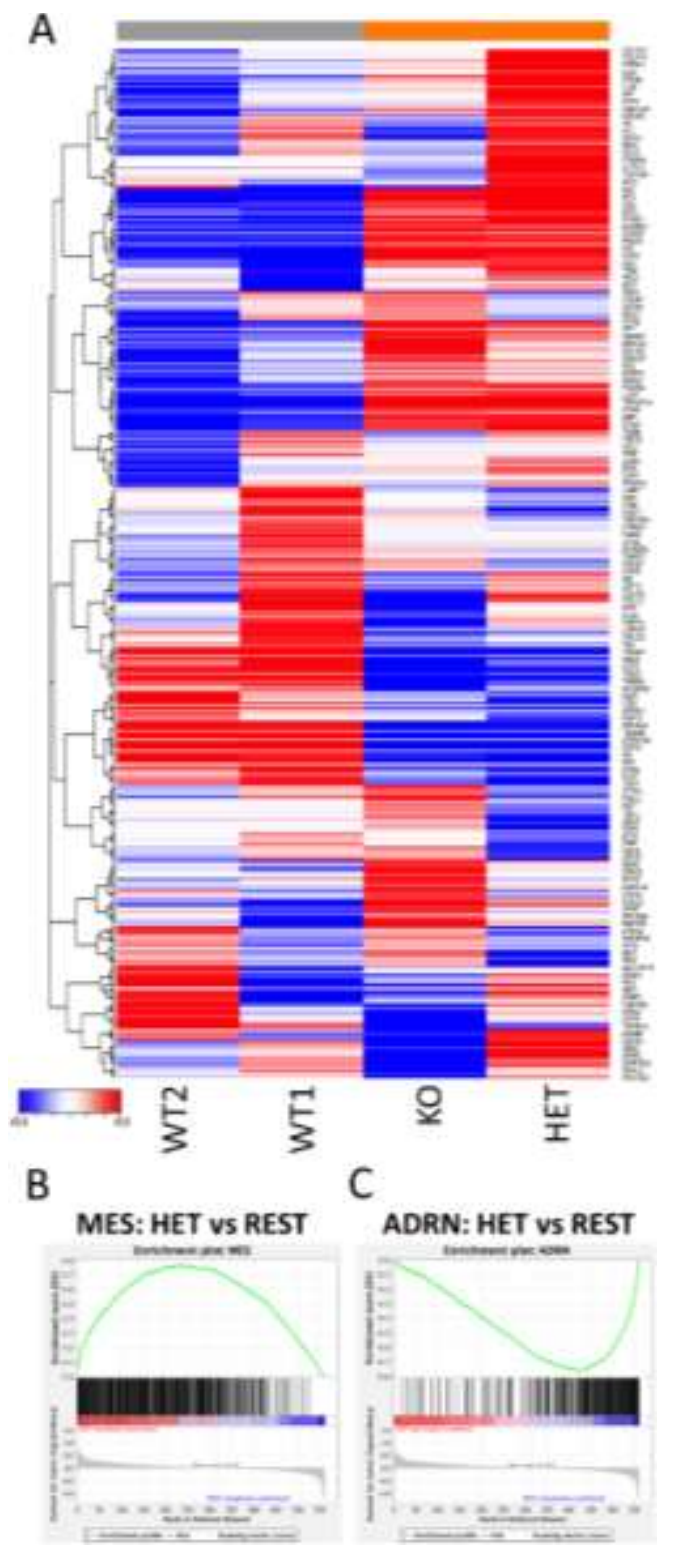

Figure 3-12. Mouse model comparison to human NBL adrenergic and mesenchymal signatures.

(A) Comparison of tumor mRNA expression profiles to ADRN and MES genes identified in NBL patient cells by van Groningen, et al. (van Groningen, 2018, Nat Gen)

(B) Gene set enrichment analysis (GSEA) of HET vs WT1, WT2, and KO tumors using the MES gene signature indicated a significant correlation between HET and MES genes. (C) GSEA of HET vs WT1, WT2, and KO tumors compared to ADRN gene signature indicated a significant correlation between HET and ADRN genes.

Data Source: van Groningen, T., Kroster, J., Valentijn, L. J., Zwijnenburg, D. A., Akogul, N., Hasselt, N. E., ... Versteeg, R. (2017). Neuroblastoma is composed of two superenhancer-associated differentiation states. Nature Genetics.

https://doi.org/10.1038/ng.3899 


\section{Discussion}

Our study focused on the functional testing and characterization of a genotype that has been posited for over 30 years as a detrimental combination in HR NBL (Attiyeh et al., 2005; G M Brodeur, 1995; Garrett M. Brodeur, 2003; García-López et al., 2020; Sausen et al., 2013; Schwab, Praml, \& Amler, 1996). We generated a novel mouse model of HR NBL by OE of Mycn and stepwise deletion of the putative 1p36 TSG, Aridla, in the NCC progenitor cells that give rise to this disease. Through this method, we asked if Aridla is a driver mutation in MYCN amplified NBL. We causally demonstrated that in our in vivo tumorigenesis model, Aridla heterozygous loss synergizes with MYCN driven oncogenesis, which is consistent with the 1p36 LOH and MNA genotype found in $\geq 15 \%$ of HR NBL patients (Attiyeh et al., 2005; G M Brodeur, 1995; Garrett M. Brodeur, 2003; Caron et al., 1995, 1996; Davidoff, 2012; C. T. Fong et al., 1989; Jiang et al., 2013; Maris et al., 1995; Nakagawara et al., 2018). Tumor histology analysis, in vitro differentiation studies, and RNA-seq characterization of Aridla mutant samples were consistent with an Aridla dependent block in differentiation resulting in altered cell identity and with developmental literature describing Aridla mutations in a variety of cell types (Chandler \& Magnuson, 2016; Han et al., 2019; Lei et al., 2015). Furthermore, we extended the current literature findings by defining an Aridla-dependent gene signature in our Mycn-driven NBL model.

Haploinsufficient Aridla is a driver mutation that collaborates with $M Y C N$ in NBL initiation. Epigenetic regulators have been highlighted as the most frequently mutated genes across pediatric cancers (Gröbner et al., 2018; Ma et al., 2018). Furthermore, epigenetic complexes, such as SWI/SNF are known regulators of cell identity (Lu \& Allis, 2017). Disruptions in the complexes and their critical components have been shown to subsequently disrupt normal progenitor commitment and differentiation and to even initiate cancer in some instances (B. G. Wilson et al., 2010). Pediatric cancer studies have collectively identified cell identity alterations to be a hallmark of pediatric tumors, including solid tumors such as NBL (Flavahan et al., 2017; Lawlor \& Thiele, 2012). Our tumor studies indicate that Aridla heterozygous loss is sufficient to increase the transformation kinetics of NCCs driven by Mycn. Only HET tumor groups demonstrated a consistent reduction in tumor latency. This finding is consistent with the phenotype expected of the TSG residing in the region of $1 \mathrm{p} 36 \mathrm{LOH}$ that is correlated to MNA. Large segmental gains and losses of chromosomes have made the identification of specific gene mutations difficult in NBL (Pugh et al., 2013). As stated by Pugh, et al "The notable lack of precisely defined genomic causes of this highly aggressive pediatric neoplasm reinforces the need to understand the interplay of host genetic factors, somatic mutations, chromosomal abnormalities and epigenetic alterations in the context of nervous system development." The increase of large NBL patient cohorts sequenced using WGS has identified rare point mutations that can be functionally tested (Sausen, et. al, 2014, Nat gen). We have worked to contribute a precise gene combination evaluated in the correct context that we hope will help address this issue. Furthermore, these data connect the concept of $1 \mathrm{p} 36 \mathrm{LOH}$ to the transformative events caused by loss of a specific gene in this locus. 
Aridla deletions cause a significant block in differentiation. The etology of MYCN-driven NBL has been linked to possible shifts in NCC identity during development (Huber, 2015; Tsubota \& Kadomatsu, 2018). To broadly test this idea in our model, we used in vivo and in vitro methods to study NCCs as they transformed, and differentiated, respectively. In vivo, HET and KO tumors included undifferentiated, poorly differentiated, and differentiating cell types throughout tumors as determined by pathological review. Neuronal differentiation in tumors was substantially affected by Aridla loss in histological analysis of differentiation using Tuj1 as a molecular marker. In vitro analysis of neuronal differentiation studies in primary NCCs with Aridla partial or full ablation also revealed a statistically significant blocks in neuronal differentiation. Aridl $a$ has a demonstrated role as a necessary component of SWI/SNF during cardiac, SNS, and hematopoietic development in mouse models with homozygous deletions (Gao et al., 2008; Han et al., 2019; M. R. Wilson et al., 2019) and hematopoietic development in one heterozygous experiment (Han et al., 2019). Our model fits with the current literature and highlighted an additional role for Aridla in SNS lineage differentiation into neurons and glial cells. These data suggest that NCCs display a broader sensitivity to loss of Aridla during development than previously expected.

The mesenchymal gene signature in Aridla HET tumors is consistent with an NBL MES signature derived from patient tumors. One theme consistent throughout Aridla developmental and cancer studies, is the ability of Aridla to regulate EMT-related phenotypes (Sun, Wang, et al., 2017; S. C. Wang et al., 2019). EMT is both a transition state during development and an initiation step of metastasis. We compared our tumor gene sets to two models published recently. The first was a developmental model that developed lists of the master regulators of each step during trunk NCC maturation using single cell sequencing throughout early SNS development. Using this the gene identified in this study as NCC master regulators, the HET samples demonstrated a partial block in differentiation characterized by an increase in Prrx1, a master regulator of mesenchymal identity. Two additional genes that induce migration and mesenchymal identity, Twist1 and Meox1, were upregulated in both HET and KO tumors. However, KO tumors also demonstrated an increase of the sensory glia inducer Pouf41, and two neural plate boarder specifiers, Zic3 and Olig3 that were not up regulated in HET tumors. We interpret this suggest a more immature gene signature in KO relative to HET tumors that is supported by the significant block in differentiation identified by IHC, neuronal differentiation IF studies, and significant Wong embryonic stem cell core signature identified by GSEA. We found it interesting that $\mathrm{KO}$ did not confer a tumorigenic advantage to NCCs and consider the data to collectively suggest a window of transformation during NCC progenitor differentiation that left HET NCCs extremely vulnerable to $M Y C N$-driven transformation. Gene signature analysis indicates that HET tumors are blocked in a state that significantly overlaps with the patient-derived mesenchymal signature indicative of HR NBL. Furthermore, the remaining tumor groups display a gene signature suggestive of the ADRN signature identified by van Groningen, et al (van Groningen et al., 2017). One view describing the effects of epigenetic deregulation in cancer points to deregulated cell fate as a tumorigenic consequence of epigenetic mutations (Flavahan et al., 2017).This is consistent with large pan-pediatric 
cancer studies that identified cell identity as the top alteration consistently identified across all pediatric cancers (Gröbner et al., 2018; Ma et al., 2018).

Here, we have built a novel option for future testing of fundamental mechanistic questions, as well as tools for high-throughput drug screening and biomarker development. In the context of our findings, we found that there are two limitations of this study. The first that was partially addressed and warrants further study is the concept of tumor heterogeneity. Our histological evaluation of tumors identified populations with unique differentiation statuses, however, single-cell RNA-seq would be necessary to evaluate the distribution of cell types comprising the tumor. Our findings support continuing this path in future studies. Second, a key principle of tumor stem cells that was not evaluated here is relapsed disease following a clinical treatment regimen. Considering that our study focused on the initiation properties of Aridla loss, we cannot exclude the possibility that Arid1a heterozygous and full ablation may have functional consequences in the context of disease relapse, therefore, the low frequency of homozygous Aridla mutations identified in NBL patients is worth noting. One NBL patient tumor screen identified that 3 patients with 1p36 LOH also harbored point mutations on the second allele of Aridla (Sausen et al., 2013). The point mutations were expected to result in premature protein termination functional loss of Aridla protein (Sausen et al., 2013). It is unclear if these were driver or passenger mutations and our results suggest the later. However, further characterization is necessary to determine if full Aridla ablation contributes to any tumor relapse characteristics. Despite these limitations we believe our work provides a springboard for future NBL studies. For example, an interesting addition to this model would be to include 17q gain. Chromosomal translocation and gain of chromosomal material at this locus is the most common genetic lesion in NBL patients and also correlates with 1p36 LOH and MNA tumors (Garrett M. Brodeur et al., 2014; Tolbert \& Matthay, 2018). The prevalence of $17 \mathrm{q}$ gain in $50-80 \%$ of patient tumors suggests that this region may confer additional tumor characteristics and is an important lesion to model in future studies (Garrett M. Brodeur, 2003; Davidoff, 2012).

We interpret the Arid1a-dependent phenotype identified by our study to be linked to cell identity, which is plastic in progenitor cells during development and can lead to oncogenic adaptations of cell fate (Flavahan et al., 2017; J. N. Wu \& Roberts, 2013). In a recent pan-cancer study, pediatric cancers were reported to have $45 \%$ overlap with the driver genes reported in pan-adult cancer studies (Ma et al., 2018). Currently, Aridla has been causally studied in developmental, and adult cancer mouse models, and defined as a context specific TSG (Chandler \& Magnuson, 2016; Gao et al., 2008; Han et al., 2019; Mathur et al., 2017; Sun, Wang, et al., 2017). Consistent with this concept, our model identified both similarities and differences to Aridla mutated adult cancers. Our model combines aspects of adult cancer studies, the ARIDIA field, and developmental biology to focus on the role of Aridla as a driver mutation in trunk neural crest transformation into the pediatric cancer NBL. Our findings in this novel mouse model of NBL support the hypothesis that Aridla is a haploinsufficient TSG that synergizes with MNA to disrupt lineage development programs, resulting in increased oncogenesis due to altered cell identity and furthermore, is a model consistent with NBL patient tumors by gene 
expression analysis. The results presented in this body of work highlight the importance of context specific disease modeling, especially in high-mortality orphan diseases such as this pediatric cancer. Therefore, this study functionally expands not only the NBL field's understanding of the consequences of deregulated epigenetic programs in pediatric cancer, but also provides higher resolution and connectivity to 25 cancer types by furthering our understanding of the most mutated member of the SWI/SNF epigenetic complex. 


\section{CHAPTER 4. ARIDIA AND DNA DAMAGE}

\section{Introduction}

Aridla is a known regulator of cell cycle. ARID1A has been shown to bind to the MYC promoter, and subsequently regulate p21 expression, a target of C-MYC (Nagl et al., 2005; Nagl, Zweitzig, Thimmapaya, Beck, \& Moran, 2006). Furthermore, deactivating $A R I D 1 A$ and $P 53$ mutations are mutually exclusive across many cancer types, and $A R I D I A$ mutations are found in cancers with multi satellite instability, while P53 mutations are not (Kadoch et al., 2013; Mathur, 2018; R. C. Wu et al., 2014). This suggests a partial overlap of $A R I D 1 A$ and P53 functions. Recently, two independent groups published studies implicating ARID1A as a regulator of the DNA damage response following double-stranded DNA damage through its binding partner ATR (Lord et al., 2016; Shen et al., 2015). ATR induces phosphorylation of chk-1 causing cell cycle arrest and apoptosis (Goto, Kasahara, \& Inagaki, 2014). When ARIDIA was homozygously deleted in colorectal cancer line HCT116, the cell cycle checkpoint was impaired, and cells were sensitized to PARP inhibitor (Shen et al., 2015). In the same cell line, an additional study was published implying that $A R I D 1 A$ knockout cells impaired the DNA damage response by ATR, which no longer transmitted the DNA damage response through p-chk1(Lord et al., 2016). More recently, a third study was published using "other" cell lines with shRNA mediated knockdown of ARID1A and ARID1A depletion was strongly correlated to resistance in 496 cancer cell lines, but most notably in sarcoma lines (Hu et al., 2018).

\section{Materials and Methods}

\section{Proliferation assays and drug treatment}

Tumor derived cell lines were plated in fibronectin coated 96-well plates at 6000 cells per well in IMDM $+10 \%$ FBS. Three hours after plating, the drugs were added at the indicated concentrations and incubated for 72 hours at 5\% CO2 until analysis. At the experimental end point, CyQuant direct fluorescent cell detection reagent (Thermo Fischer) was added to wells and incubated for 1 hour at $37^{\circ} \mathrm{C}$. Data was imported into GraphPad Prism and

\section{Cell lines}

Neuroblastoma cell lines SK-N-AS, SY5Y, SK-N-SH, SK-N-DZ, Kelly, IMR-32, and SK-N-BE2 were purchased from American Type Culture Collection (ATCC) and cultured according to manufacture instructions. A table listing a summary of genetic information from Harenza, et al. for MYCN and 1p36 status in common neuroblastoma cell lines are listed in Table A-1 (Harenza et al., 2017). 


\section{Histology analysis}

Tumors were fixed in 10\% neutral buffered formalin, embedded in paraffin, sectioned at $4 \mu \mathrm{m}$, stained with hematoxylin and eosin (HE). All formalin-fixed, paraffin-embedded (FFPE) tissues were sectioned at $4 \mu \mathrm{m}$, mounted on positive charged glass slides (Superfrost Plus; Thermo Fisher Scientific, Waltham, MA), and dried at $60^{\circ} \mathrm{C}$ for 20 minutes. The IHC procedures and antibodies used for mouse tissue to detect protein markers are listed in Table A-2. All HEs and IHCs were reviewed by light microscopy and interpreted by a board-certified veterinary pathologist (HT). IHCs were scanned at a 20x (objective lens) magnification using an Aperio ScanScope XT scanner. Immunoreactivity was quantified as a weighted IHC score (0-300) using a modified version of the Color Deconvolution algorithm and ImageScope software (Leica Biosystems).

\section{Immunoblotting}

Samples were collected on ice by washing $1 \mathrm{x}$ with PBS, then adding RIPA buffer (Thermo Scientific, PI89900) and 2x Halt Protease Phosphatase inhibitor. Samples were scraped into a tube, vortexed for 10 seconds and lysed for 20 minutes rotating at $4^{\circ} \mathrm{C}$. Samples were sonicated for 10 second intervals totaling 30 seconds, then lysates were cleared by centrifugation. Samples were incubated for 30 minutes in 1x SDS-loading buffer with 9\% BME and 50ug of total lysate was separated by gel electrophoresis on 4$20 \%$ tris-glycine gels (BioRad \#4561094) at 300V for 25 minutes. Samples were transferred according to protein size. Membranes were blocked for 1 hour at room temperature (RT) in PBS .1\% Tween 20 (PBST)/5\% (w/v) milk. PVDF membranes were probed with primary antibodies incubated $\mathrm{o} / \mathrm{n}$ at $4^{\circ} \mathrm{C}$ with gentle shaking. Next, membranes were washed with PBST and incubated with secondary antibody (1:4000, Cell Signaling Technologies) in PBST/5\% (w/v) milk for 1 hour at RT. Membranes were washed in PBST and protein signal was detected using chemiluminescent substrate (Genesee Scientific \#20-302). Antibody concentrations and protein sizes reference Table A-3.

\section{Neural crest cells}

NCCs with Arid1a modifications were subjected to increasing amounts of Mycn virus (Addgene \#35394). Samples were harvested 72 hours following exposure to virus, processed using the Qiagen RNeasy kit according to the manufacturer's protocol. cDNA was synthesized, and qPCR was performed with Taqman primer/probe sets for Mycn and Aridla. Ppib was used as the house keeping gene. Reads were normalized to the internal control (Ppib), and reported as changes in expression relative to the control group.

Statistical analysis was used to test differences in Mycn expression between the two groups, or Aridla differences between the two groups.of NCCs. Statistical analysis was 
performed under the following parameters: RM two-way ANOVA with the GeisserGreenhouse correction, matched values are spread across a row. Sidak's multiple comparisons test, with individual variances computed for each comparison using GraphPad Prism 8.2.1 (441).

\section{Annexin V analysis}

Statistical analysis was completed under the following parameters: Discovery determined using the Two-stage linear step-up procedure of Benjamini, Krieger and Yekutieli, with $\mathrm{Q}=1 \%$.Each row was analyzed individually, without assuming a consistent SD. Number of $t$ tests: 5 using GraphPad Prism 8.2.1 (441).

\section{Results}

\section{ARID1A protein levels are increased in MNA cell lines}

We were interested to evaluate relative protein levels of N-MYC and ARID1A in 1 p36 deleted cell lines and in control cell lines, and to determine if expression of N-MYC and ARID1A were as expected. The cell lines chosen for analysis had been analyzed previously by sequencing studies that confirmed the chromosomal changes (Harenza et al., 2017). We controlled this study by including three non-MNA/1p36 cells, one MNA cell line, and next compared four cell lines with $1 \mathrm{p} 36$ and MNA. Interestingly, the cell lines with $1 \mathrm{p} 36 \mathrm{LOH}$ did not exhibit an obvious expression decrease in ARID1A, even though the genetic material was lost (Figure 4-1A). Furthermore, the only cell line examined with MNA and WT 1p36 expressed increased levels of Arid1 a protein relative to NBL cell lines without MNA (Figure 1A). This suggested that MNA causes an ARID1A expression response. To validate this result in primary NCCs, MYCN was titrated into WT1, HET, WT2 and KO primary cells in a range of MOI 0-3 (Figure 4-1C and D). Mycn and Aridla levels were validated by qPCR. Consistent with our previous results, Aridla expression increased as MYCN expression increased at a MOI of 2 and 3 in WT1 and an MOI of 2 in HET NCCs (Figure 4-1C and D). This MYCN-dependent increase of Arid1a expression suggested that Aridla behavior in NCCs modeled the expression of ARID1A in NBL cell lines. Next, we monitored cell death in response to MYCN OE. As before, we transduced the cells with an increasing amount of Mycn virus (MOI 0-3) and submitted the cells to annexinV flow-cytometry analysis at 72 hours posttransduction (Figure 4-2A and B). In this study, no significant differences in cell death were identified between controls and Aridla deleted samples. Taken together, this data suggests a regulatory connection between $M Y C N$ and ARIDIA, however our data did not support the concept that Aridla loss alters NCC cell death in response to $M Y C N$ expression. 


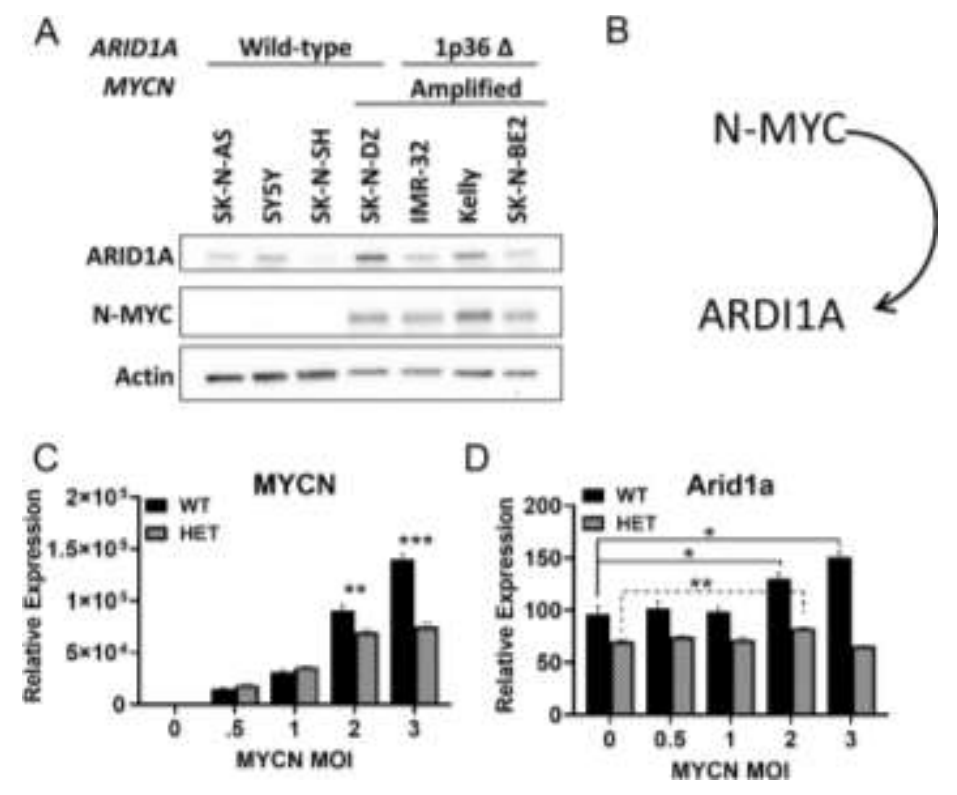

Figure 4-1. N-MYC and ARID1A protein expression are correlated in NBL and NCC samples.

(A) Panel of NBL cell lines with known chromosomal alterations analyzed for ARID1A and N-MYC protein expression by western analysis.

(B) Schematic of proposed regulation of ARIDIA by N-MYC.

(C and D) qRT-PCR results from Aridla wild-type and heterozygous NCCs transduced with increasing amounts of Mycn virus.

(C) Mycn mRNA was significantly increased in WT1 compared to HET NCCs in samples transduced with a MOI of $2(* * p=0.003)$ and MOI of $3(* * * p=0.00005)$.

(D) To determine if Mycn expression correlated to Aridla expression, a 2way ANOVA with multiple comparisons was run to evaluate Aridla expression relative to its MYCN MOI 0 control. Aridla expression in WT1 samples significantly increased in response to increasing amounts of MYCN at MOI $2 * p=0.026$ and MOI $3 * p=0.01$. Aridla HET demonstrated a significant increase at MOI $2 * * p=0.002$ when compared to HET at MOI 0. $\left({ }^{*} p \leq 0.05, * * p \leq 0.005, * * * p \leq 0.0005\right)$. Representative results of $\mathrm{n}=3$ biological replicates. 

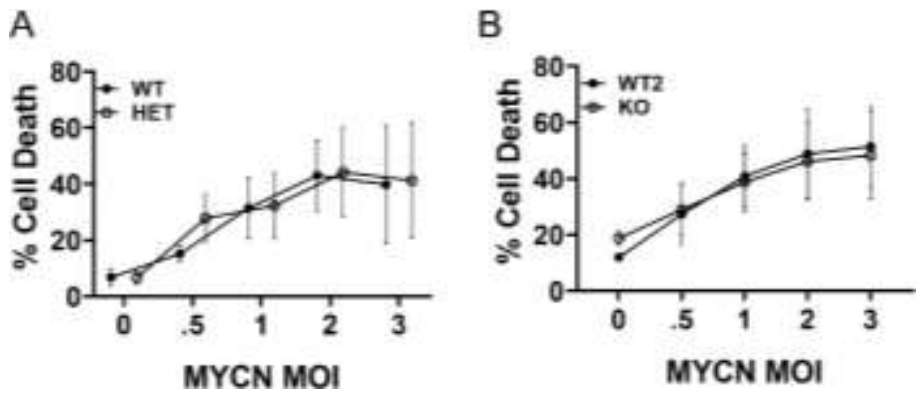

Figure 4-2. Aridla status was not correlated to apoptotic response when Mycn was titrated into primary NCCs.

(A and B) Annexin V and DAPI were used to determine fractions of early and late apoptotic and dead cells by flow cytometry. No statistical differences were observed between WT1 and HET, or WT2 and KO at any MOI tested. 


\section{Characterization of DNA damage and cell death in end-stage tumors}

Aridla loss has been shown to increase mismatch repair deficiency and mutagenesis (Shen et al., 2018). One possible reason HET NCCs transformed more readily than WT1 might be explained by DNA damage repair deficiency resulting in an accumulation of genetic mutations, reduced DNA damage signaling, and decreased cell death relative to controls. Therefore, we analyzed end stage tumors for signs of a mutator phenotype (Loeb, 2001; Shen et al., 2018). We expected tumors with a possible mutator phenotype to demonstrate a low rate of $\gamma \mathrm{H} 2 \mathrm{ax}$ induction, and low levels of cleaved caspase 3 relative to controls. This would suggest possible genomic instability, and point to a potential TSG caretaker role for Aridla in our model (Shen et al., 2018; R. C. Wu et al., 2014). To this end, we evaluated $\gamma \mathrm{H} 2 \mathrm{ax}$, an upstream DNA damage sensor, and cleaved Caspase-3, a downstream mediator of cell death by IHC in end point tumors (Shen et al., 2018, 2015). Interestingly, no significant differences were observed in HET samples (Figure 4-3A and B). KO samples indicated an increase in cleaved caspase-3 relative to controls, but no difference in $\gamma \mathrm{H} 2 \mathrm{ax}$ (Figure 4-3C and D). Therefore, we did not observe leading indicators of a mutator phenotype or genomic instability in Aridla depleted tumors in our model. This suggests that if impaired DNA damage response is involved in NCC transformation, it was not evident in the end-stage tumors.

\section{Aridla heterozygous and homozygous tumor derived cell lines do not have impaired ATR signaling}

ARID1 A has been shown to bind p53 and to interact with ATR to induce DNA damage signaling (Guan et al., 2011; Lord et al., 2016; Shen et al., 2015). We reasoned that if Aridla is involved in the DNA damage response, impaired DNA damage signaling may be involved in our model of tumorigenesis. We did not see an increase of DNA damage using $\gamma \mathrm{H} 2 \mathrm{ax}$ as a readout in in any tumor groups evaluated and considered that one reason may be that a reduced DNA damage response may be evident during early stages of NCC transformation but missed at end point analysis. Therefore, we decided to induce DNA damage and measure the consequences in tumor derived cell lines (TDCLs).

Due to the emerging data indicating Aridla-ATR interactions (Lord et al., 2016; Shen et al., 2015), we decided to induce DNA damage in TDCLs to see if Aridla deletions affected ATR signaling, and altered drug resistance. We chose topotecan and etoposide, two front line chemotherapeutics used in the COG treatment regimen, to induce DNA damage in tumor derived cell lines (TDCLs) (Pinto et al., 2015). First, a dose response was established in three TDCLs for each genotype. Next, TDCLs (three per genotype) were treated with both compounds, collected across time points and probed for key mediators of the DNA damage response. Phosphorylated chk1 was used as a readout of ATR-mediated DNA damage. Chk1 is an effector protein that is phosphorylated by p-ATR, and transduces the DNA damage signal downstream to p53 (Balmus et al., 2019). 

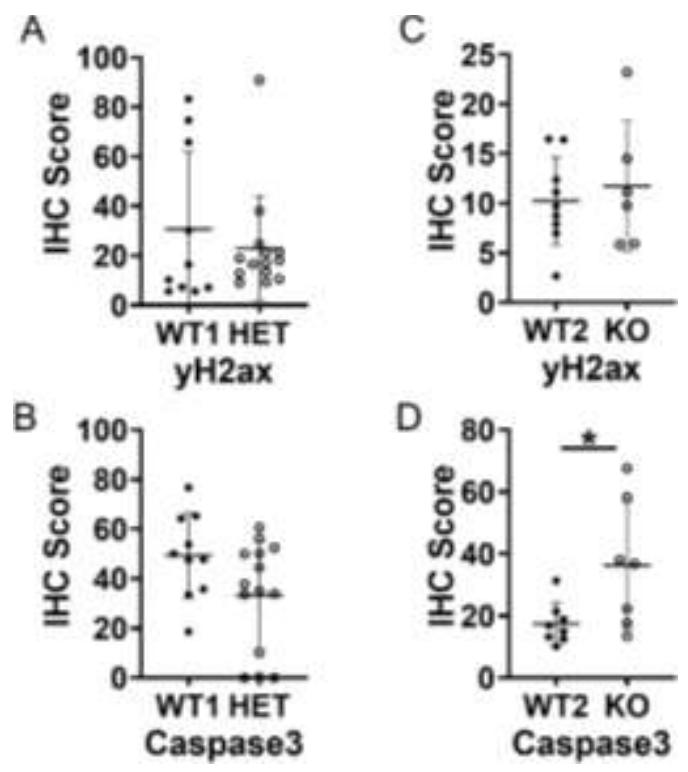

Figure 4-3. DNA damage and cell death analysis of tumors. (A and B) Quantification of WT1 vs HET tumors at endpoint following IHC (A) for the DNA damage response protein $\gamma \mathrm{H} 2 \mathrm{ax}(\mathrm{WT} 1 \mathrm{n}=9$, HET $\mathrm{n}=13)$ ns. $p=0.744$ and (B) the cell death mediator Caspase3 (WT1 $\mathrm{n}=9$, HET $\mathrm{n}=13$ ) ns. $p=0.209$ as analyzed by color deconvolution analysis. Points represent staining and quantification from individual tumors. (C and D) Quantification of WT2 vs KO tumors at endpoint following IHC (A) for the DNA damage response protein $\gamma \mathrm{H} 2 \mathrm{ax}(\mathrm{WT} 2 \mathrm{n}=9, \mathrm{KO} \mathrm{n}=6) \mathrm{ns} . p=0.955$ and

(B) the cell death mediator Caspase $3(\mathrm{WT} 1 \mathrm{n}=8, \mathrm{KO} \mathrm{n}=7) * p=0.029$ as analyzed by color deconvolution analysis. Points represent staining and quantification from individual tumors.

$p$ values were calculated using Mann Whitney test; $(* p \leq 0.05)$. 
As a dsDNA damage control, we used $\gamma \mathrm{H} 2 \mathrm{ax}$, an upstream transducer of DNA damage. HET lines did not demonstrate therapeutically relevant resistance to etoposide or topotecan at any dose evaluated (Figures 4-4A and 4-5A). Similarly, KO lines were not significantly resistant or sensitive to either drug relative to WT2 TDCLs (Figures 4-4B and 4-5B). Our study did not demonstrate a relationship between Aridla loss and deficient ATR signaling at the time points evaluated for etoposide or topotecan induced DNA damage in HET samples relative to WT1 control TDCLs (Figures 4-4C and 4-5C), nor in KO TDCLs compared to WT2 control lines (Figures 4-4D and 4-5D). To determine if our TDCL results were consistent with ATR-mediated DNA damage response in NBL samples, we evaluated human NBL cell lines for signs of impaired ATR signaling (Figure 4-6). We treated cells with increasing amounts of topotecan, and evaluated the samples following six hours of treatment. Although DNA damage was induced, no striking differences in ATR signaling were evident in cells with 1p36 LOH and MNA (Kelly) relative to MNA only control cells (SK-N-AS) (Figure 4-6).

Taken together, the differences in tumorigenic advantage in HET tumors were not explained by the ATR mediated DNA damage response. Aridla has been implicated as a modulator of lineage commitment in mouse embryoid bodies (Gao et al., 2008). Considering these results, we hypothesized that rather than the DNA damage-mediated proliferative advantage commonly seen in adult cancers, a developmental aberration may result in a tumorigenic advantage in our pediatric cancer model.

\section{Discussion}

MNA upregulated ARID1 a protein levels in NBL cell lines. Additionally, Aridla was slightly increased at the mRNA level following the titration of a Mycn OE virus. ARIDIA mutations and MYC amplification co-exist in a multiple cancers (Giulino-Roth et al., 2012; Kim et al., 2018; C. Love et al., 2012; Muzny et al., 2012; Sun, Wang, et al., 2017; Witkiewicz et al., 2015). This suggests that ARID1A, and possibly other BAF family members, collaborate with MYC family oncogenes in many cancers, although these synergies may present with cancer specific phenotypes. Furthermore, the regulatory circuits controlling Arid1a have not been established. Further testing to determine if MNA regulates $A R I D 1 A$ may provide insights into other cancers with synergy between a transcription factor oncogene and a chromatin remodeling tumor suppressor. This link may also provide details into broader regulation of SWI/SNF and its regulatory partners. Our data suggest that further experiments using ChIP-PCR or ChIP-seq to determine if N-MYC binds to the ARIDIA promoter would provide insight into the mechanism of $A R I D 1 A$ regulation.

Mycn OE did not induce differential apoptosis in HET and KO primary cells. TSGs are classified as either caretakers or gatekeepers (Shen et al., 2015; R. C. Wu et al., 2014). Caretakers comb the DNA to identify DNA damage and signal repair, and gatekeepers regulate proliferation and apoptosis (R. C. Wu et al., 2014). 
A

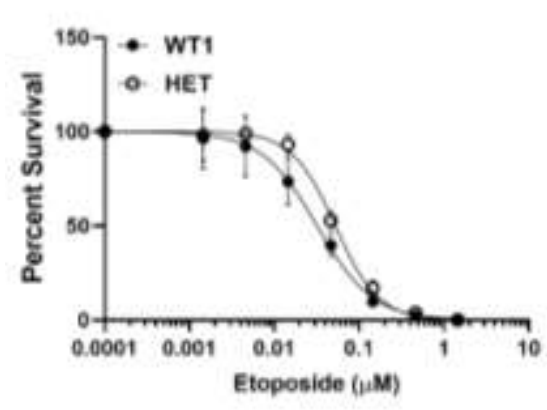

C

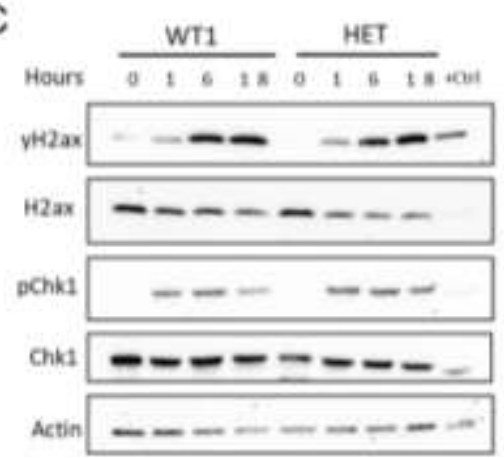

B

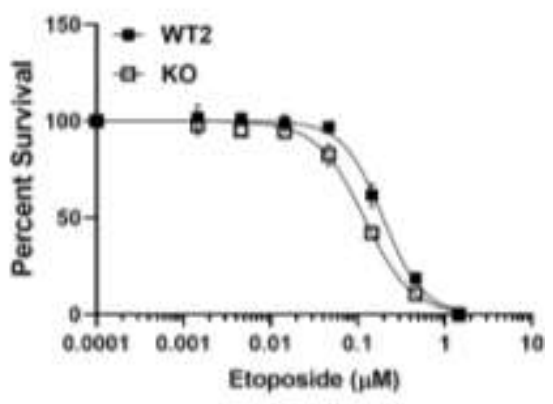

D

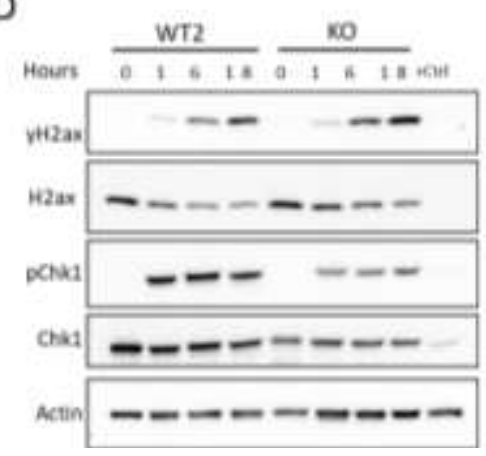

Figure 4-4. Aridla HET and KO tumor derived cell lines do not have impaired ATR signaling in response to etoposide treatment.

(A and B) Tumor derived cell lines ( $\mathrm{n}=3$ per genotype) were treated with etoposide in increasing half-log doses for 72 hours. Percent survival was measured by CyQuant $(n=3)$. Best curve fit and $\mathrm{EC}_{50}$ and $\mathrm{AUC}$ were calculated and determined to be statistically insignificant.

(C and D) Representative western blots indicating ATR-dependent DNA damage response activation following treatment with either etoposide $(10 \mu \mathrm{M})$. Experiments were repeated in three independent cell lines for each genotype.

(A and C) WT1 or HET TDCLs; (B and D) WT2 or KO TDCLs treated with etoposide. $\mathrm{EC}_{50}=$ half-maximal response, $\mathrm{AUC}=$ area under curve 
A

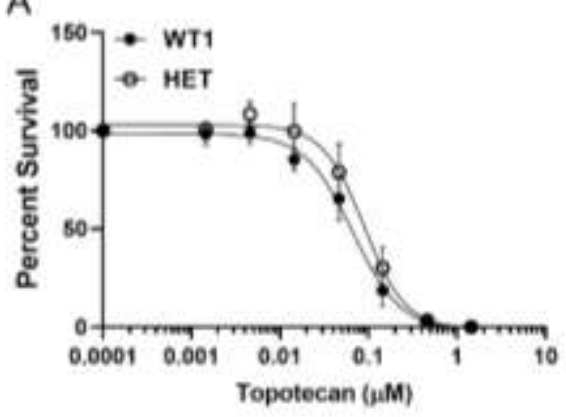

C

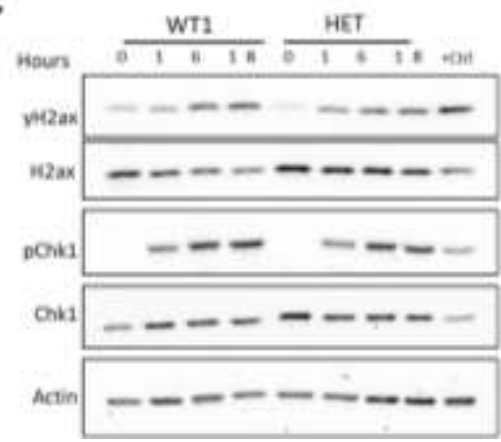

B

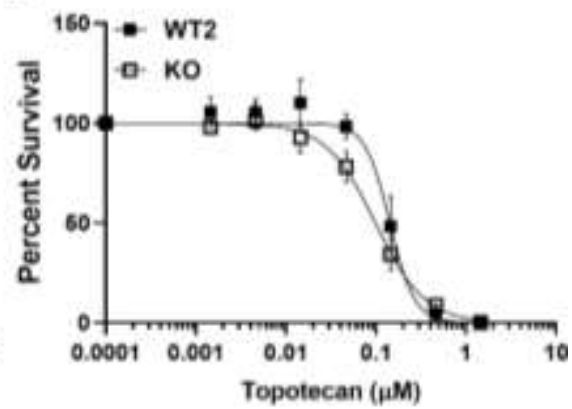

D

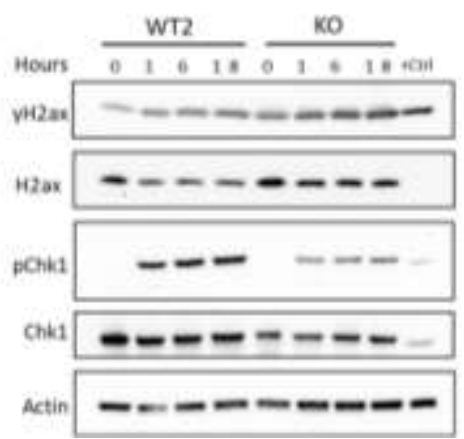

Figure 4-5. Arid1a HET and KO tumor derived cell lines do not have impaired ATR signaling in response to topotecan treatment.

(A and B) Tumor derived cell lines ( $\mathrm{n}=3$ per genotype) were treated with topotecan in increasing half-log doses for 72 hours. Percent survival was measured by CyQuant $(n=3)$. Best curve fit and $\mathrm{EC}_{50}$ and $\mathrm{AUC}$ were calculated and determined to be statistically insignificant.

(C and D) Representative western blots indicating ATR-dependent DNA damage response activation following treatment topotecan $(1 \mu \mathrm{M})$. Experiments were repeated in three independent cell lines for each genotype.

(A and C) WT1 or HET TDCLs; (B and D), WT2 or KO TDCLs treated with topotecan. $\mathrm{EC}_{50}=$ half-maximal response, $\mathrm{AUC}=$ area under curve 


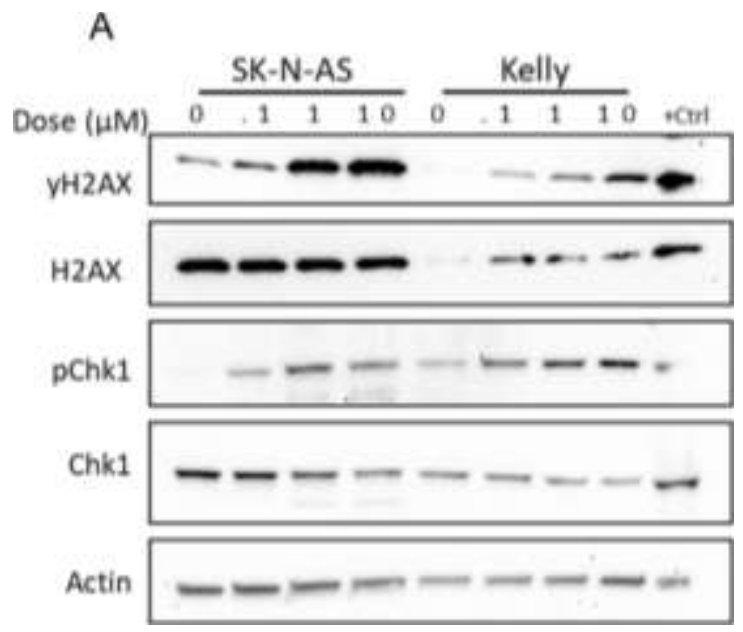

Figure 4-6. 1p36 LOH did not alter ATR signaling in topotecan treated NBL cells. (A) NBL cell lines were treated with topotecan in 10x increasing doses for 72 hours. Representative western blot indicating ATR-dependent DNA damage response activation following treatment topotecan $(\mathrm{n}=1)$. 
Aridla is a known regulator of the G2-M transition, which suggests a gatekeeper role (Shen et al., 2015). ARID1A and P53 mutations have been identified as mutually exclusive, which may suggest a role for Arid1a in the apoptotic response. Avoiding apoptosis is a hallmark of a transforming phenotype, and Aridla deletion has been shown to increase a mutability phenotype (Shen et al., 2018). Caspase 3 has also been shown to increase in Aridla KO tumors compared to controls (Shen et al., 2018). Furthermore, MNA drives DNA synthesis, which causes replication stress and subsequent apoptosis (Ham et al., 2016; Ruiz-Pérez et al., 2017). Therefore, it is possible that Aridla loss may relieve this path to cell death. It is interesting that we did not see significant differences across our primary cells.

IHC analysis of tumors did not indicate a mutator phenotype in HET tumors. Furthermore, our Caspase 3 IHC results may suggest that Aridla KO induces stress in during tumor development. One possible explanation for this result is that it suggests a necessary minimum requirement of Aridla for NCCs to thrive. Next, it is possible that differences in the DNA damage response are necessary for primary cells to establish themselves in the host tissue. Therefore, differences may not be observed in endstage tumors that have adapted to their environment. Considering this possibility, we decided to induce DNA damage in TDCLs to determine if the DNA damage response was impaired in vitro.

Aridla loss does not impair the ATR-dependent DNA damage response. The role of Aridla as a TSG is emerging, and a burgeoning field of DNA damage studies have suggested a caretaker role based on p53 and ATR experiments (Guan et al., 2011; Lord et al., 2016; Mathur, 2018; Shen et al., 2018, 2015). Furthermore, multiple impactful studies have indicated both increased resistance and increased sensitivity to chemotherapeutics in Aridl $a$ adult cancer models (Hu et al., 2018; Lord et al., 2016; Shen et al., 2015). One explanation is that these differing results may be representative of the tissue dependent role of $A R I D 1 A$, or a difference in the role of $A R I D 1 A$ loss in adult versus pediatric cancer. In the model presented here, we maintained tissue specificity while modifying Aridla dose to better understand the characteristics of ARIDIA loss in NBL. The findings of our pediatric model of NBL provides a tool and rationale for dosage and tissue controlled Aridla studies that may benefit therapeutic development efforts. 


\section{CHAPTER 5. DISCUSSION}

\section{Significance of Aridla Findings in the NBL Community}

In order to discuss the molecular mechanisms that underpin HR NBL, first we must establish an understanding of the NBL patterns that can be identified clinically from large patient cohorts.

\section{Connecting causal gene mutations and patient outcomes}

Briefly, the overarching theme of NBL patient care, is that low and intermediate risk patients have good prognosis and $>90 \%$ 5-year survival rates (Coughlan et al., 2017; Davidoff, 2012; Nakagawara et al., 2018). Surgery and or limited exposure to chemotherapy are enough to achieve these results (Coughlan et al., 2017; Davidoff, 2012). In some patients, disease spontaneously regresses without any clinical treatment. However, as previously discussed, HR NBL patients present with a disease composed of multiple compounding negative factors. Two of the most negative indicators of disease progression include heterogeneous tumors and metastatic disease at diagnosis (Garrett M. Brodeur et al., 2014; Tolbert \& Matthay, 2018). Following intensive multimodality therapy, HR patients often relapse and succumb to disease (Attiyeh et al., 2005; Garrett M. Brodeur, 2003; Davidoff, 2012; Jiang et al., 2013; Maris \& Matthay, 1999; Muzny et al., 2012; Nakagawara et al., 2018; J. R. Park et al., 2010). It is important to understand the primary event or events that link each of these issues to disease initiation and poor outcomes. However, a common thread throughout the entire NBL community is a lack of knowledge around the precise genes that initiate this disease. (Garrett M. Brodeur, 2003; Pugh et al., 2013). This results in a disconnect between causal gene mutations and resulting poor patient outcomes (Garrett M. Brodeur et al., 2014). Next, we consider one potential mechanism of NBL initiation in the context of recent literature and results from our study.

\section{Genetic mutations that disrupt NCC identity may contribute to NBL initiation}

\section{Supportive findings}

Disrupted cell identity of NCCs during SNS development is one possible route to NBL initiation (Jiang et al., 2013; Tsubota \& Kadomatsu, 2018). Clinical and causal studies have supported the concept that poorly differentiated NCCs give rise to this disease (Davidoff, 2012; Etchevers et al., 2019; Furlan et al., 2017; Jiang et al., 2013; Soldatov et al., 2019; van Groningen et al., 2017). Some of the strongest evidence in support of this argument come from clinical trials that found differentiation therapy using retinoic acid added to multimodal therapy improved patient event free survival significantly (Davidoff, 2012). Causal studies from models of $M Y C N$-driven NBL also support this argument. The first $M Y C N$-driven mouse model of NBL spontaneously 
enforces $M Y C N$ expression off of a $T H$ promoter, which leads to disrupted sympathetic neuron commitment and function and is a faithful model of human disease (Weiss et al., 1997). Two additional models generated by MYCN OE in SNS cells NBL have reproduced these results (Olsen et al., 2017; Zhu et al., 2012). Our recent addition to these causal models considers 1 and 2 copy deletions of Aridla combined with Mycn OE, resulting in reduced tumor latency in HET tumors (Figures 3-1, 3-2 and 3-3), and tumor histology and gene signatures that distinguished HET and KO tumors from Mycn OE controls (Figure 3-5). Our findings support the long standing hypothesis that NBL initiation is due to the chromosomal aberrations MNA and 1p36 LOH (G M Brodeur, 1995; G M Brodeur et al., 1977; Garrett M. Brodeur et al., 2014; Caron et al., 1995; C.

Fong, Look, Vogelstein, \& Cavenee, 1992; García-López et al., 2020; Olsen et al., 2017; Sausen et al., 2013). Our findings further support a connection between disrupted cell identity contributing to NCC transformation into NBL (Figures 3-6 through 3-12). (Etchevers et al., 2019; Furlan et al., 2017; Jiang et al., 2013; Soldatov et al., 2019).

\section{Outstanding questions}

Despite the cumulative findings that support the idea that de-regulated SNS differentiation is causal in NBL initiation, possibly through the oncogenic function of $M Y C N$, it is unclear whether de-differentiation or a block in differentiation during SNS development are the causal routes (Tsubota \& Kadomatsu, 2018). Conclusions from a recent study experimentally illustrate this point. Van Groningen, et al. found that NBL tumors were composed of cells with two unique identities (van Groningen et al., 2017). This study viewed the two cell identities to be defined and regulated by super-enhancer gene programs (van Groningen et al., 2017). This is consistent with recently published data from multiple groups (Boeva et al., 2017; Decaesteker et al., 2018; L. Wang et al., 2019). Furthermore, this study found that the mesenchymal type of NBL tumor cells (MES) were more primitive, and expressed a genetic profile that resembled NCCs (van Groningen et al., 2017). The second tumor cell group, adrenergic (ADRN), could be dedifferentiated into the MES cell type (van Groningen et al., 2017). Collectively, their data supports the hypothesis that NBL tumors are comprised of cells with plastic cell identity regulated by super enhancers (Boeva et al., 2017; Decaesteker et al., 2018; van Groningen et al., 2017; L. Wang et al., 2019). Their work further highlights the lack of clarity of whether NBL tumors result from blocked differentiation, de-differentiation, or a mixture of both features. The $T H$-driven $M Y C N$ model suggests that de-differentiation of committed SNS cells may contribute to tumorigenesis, since TH is expressed in differentiated neurons. Our findings, however, suggest a block of differentiation during NCC commitment to SNS lineages gives rise to an aggressive form of this disease. Altogether, studies published across groups and NBL cancer models suggest that cancer cell plasticity contributes to the heterogeneity of NBL. Additional insights can be gleaned from the NCC field and a detailed discussion of recent studies regarding NCC progenitor cell plasticity and cell fate can be found in the "Cell identity" section of this dissertation. 


\section{Hunt for the 1 1p36 Tumor Suppressor Gene}

In the context of intrinsic factors that contribute to disease onset and progression, one needs to understand the mechanism underlying this disease and how these are disrupted during disease onset. Therefore, the next question to consider is what are the cellular disruptions that are proposed to lead to an altered cell identity and subsequent HR disease. If we evaluate NBL genetic studies, we begin to see the supportive data linking the cellular events of this disease to the mechanisms that are suspected to cause it. Over 40 years ago, the first chromosomal rearrangement that was consistently identified in NBL patients were large deletions of 1p (G M Brodeur et al., 1977). These were found to correlate with MNA, origin in the adrenal glands, and HR disease, which has been supported by findings from multiple groups (Caron et al., 1995; C. T. Fong et al., 1989). Dr. Brodeur's findings suggested that at least one or a collection of TSGs resided in the $1 \mathrm{p}$ region of $\mathrm{LOH}$ lost in HR patients, based on the two-hit-hypothesis, the hunt for loss of function genes driving this catastrophic illness ensued (G M Brodeur, 1995; Garrett M. Brodeur, 2003; C. T. Fong et al., 1989; Maris et al., 1995; Versteeg et al., 1995; White et al., 2005). Sequencing studies mapped chromosomal alterations with increasing specificity, and causal studies systematically tested each putative gene (Maris \& Matthay, 1999; White et al., 2005). The combination of these studies converged on the smallest region of consistent deletion (SRCD), which is positioned in distal 1p36, and spans 261 kb at 1p36.3 (Maris \& Matthay, 1999; Schulte \& Eggert, 2015; White et al., 2005). Overall, these studies have concluded that the chromosomal breakpoints in 1p36 do not consistently map to one gene locus (Garrett M. Brodeur, 2003; Caron et al., 1995; Maris \& Matthay, 1999; Pugh et al., 2013). This inconsistency has complicated separating the driving chromosomal lesions from passenger events in mapping studies. Because 1p36 deletions include large regions of chromosomal loss, the remaining allele was evaluated for mutations that could result in complete loss of one gene, and suggest that it is a 1p36 TSG (Maris \& Matthay, 1999). These mapping approaches did not reveal point mutations on the second allele of any of the putative TSGs (Maris \& Matthay, 1999). To complement mapping studies, groups looked for gene and protein expression loss (Carén, Fransson, Ejeskär, Kogner, \& Martinsson, 2007; Fujita et al., 2008; Janoueix-Lerosey et al., 2004; Okawa et al., 2008). However, these studies uncovered few TSG prospects that demonstrate causal evidence in driving NBL (Garrett M. Brodeur et al., 2014).

In 2008, a bisulfite sequencing study revealed methylation on the sister allele of CHD5, a 1p36 TSG candidate (Fujita et al., 2008). Additionally, a large sequencing study identified point mutations corresponding to the TSG Aridla in 4 of 71 patients studied (Sausen et al., 2013). One of these was a single point mutation expected to cause termination of the transcript and the resulting protein product of that allele (Sausen et al., 2013). The additional three patients demonstrated a 1p36 LOH and a point mutation on the sister chromatin (Sausen et al., 2013). These mutations were expected to result in the early termination of mRNA transcript and a subsequent loss of protein expression (Sausen et al., 2013). Furthermore, the CHD5 findings and ARIDIA findings are consistent with an earlier study that proposed two TSG regions in 1p36, one distal region that was independent of MNA disease, and a larger proximal region that correlated to 
MNA (Caron et al., 1995). Taken together, these results supported further investigation of CHD5 and ARIDIA.

Further investigation of CHD5 in causal studies indicated that deletion of this gene did not synergize with Mycn-driven disease but may be a contributor to tumors containing 1p36 LOH without MNA, which correlates to a patient cohort with favorable outcomes (García-López et al., 2020; Maris \& Matthay, 1999). Consistent with the study by Sausen et al., one study found that in $M Y C N$-driven cancer during a tumor evolution study, the tumor bulk maintained Aridla deletions, but not Chd5 deletions, which strengthened the evidence for further testing of $A R I D 1 A$ deletions in $M Y C N$-driven causal studies of NBL (García-López et al., 2020).

In Chapter 3 figures 1 and 2, we move the field forward by casually testing Aridl $a$ as a driver in NCC transformation into NBL. Our study provides a comprehensive analysis of Aridla as a driver mutation. To rigorously test the hypothesis that a haploinsufficient gene loss collaborates with MYCN to drive NBL, we genetically modified Aridla by deleting none, one, or both alleles of Aridla before enforcing Mycn $\mathrm{OE}$ in untransformed primary NCCs (Figure 3-1A). Biological studies from different $\mathrm{NCC}$ isolations reproduced the result that heterozygous Aridla synergized with Mycn $\mathrm{OE}$ to initiate NBL (Figures 3-1B through E, 3-2A and 3-3A). Therefore, we propose that our findings connect the proximal 1p36 LOH identified in MNA patients to a specific gene that functions as a haploinsufficient TSG, and these findings furthermore support Aridla's candidacy as a proximal 1 p36 LOH TSG that correlates with MNA.

\section{Collaborative $A R I D 1 A$ and $M Y C N$ gene expression programs}

Not only is it important to test cooperating mutations, but it is important to validate that mouse models of cancer are faithful to human disease. Previous studies published in a comparative model found that tumors resulting from MYCN-driven transformation of primary NCCs molecularly and by pathology recapitulates NBL (Olsen et al., 2017). To characterize the model developed in this study and identify disrupted signaling events, we analyzed tumors by histology and mRNA sequencing analysis. We thought that characterizing the consequences of Aridla and Mycn alterations would highlight disruptions that correlate to findings in HR NBL patients. To this end, we investigated the gene signature resulting from sixteen tumors ( $n=4$ per group). An unsupervised hierarchical clustering analysis mapped the genes differentially expressed in these four groups (Figure 3-5A). As expected, the controls from both WT1 and WT2 groups clustered together. Additionally, there are obvious differences in gene expression in the HET compared to WT1 and KO compared to WT2 (Figure 3-5A). Subsequently, we were able to identify genes that are sensitive to Arid1a dose (Figures 3-5A and 3-8). These findings will be reviewed and discussed thoroughly in the upcoming sections.

In the past, a lack of identification and causal testing of precise genes and gene combinations that initiate this disease has prevented downstream gene signature analysis and therapeutic development (Garrett M. Brodeur et al., 2014). This was the result of 
inconsistent chromosomal deletions, lack of guiding point mutations, and a lack of the scientific tools, such as bisulfite and high-throughput sequencing, needed to find these genetic events. Our identification of a haploinsufficient TSG fits into the NBL field's expectations of the proximal $1 \mathrm{p} 36 \mathrm{TSG}$, however it is an unconventional finding overall. Finally, we consider these findings as a step toward identifying gene targets and subsequent therapeutic testing and development with the aim of eventually improving patient care and outcomes. Only through identifying the precise genes, their signaling partners, and cellular consequences can we begin to build small molecule inhibitors (SMIs) to target the causal molecular abnormalities underlying NBL initiation. The remainder of this discussion will focus on the gene expression profiles and cellular consequences found in our initial tumor study.

\section{The oncogenic role of $M Y C N$}

$M Y C N$ is the oncogenic driver of HR NBL and has been connected to tumorigenic cellular events, such as increases in proliferation and cell stemness (Rickman et al., 2018; Ruiz-Pérez et al., 2017). However, as described previously, the majority of MNA is present in combination with 1p36 LOH (Garrett M. Brodeur, 2003; Caron et al., 1995; C. T. Fong et al., 1989; Jiang et al., 2013). Often these two mutations are combined with 17q gain (G M Brodeur, 1995; Garrett M. Brodeur, 2003; Davidoff, 2012; Jiang et al., 2013; Maris \& Matthay, 1999; Maris et al., 1995; Pugh et al., 2013). The synergizing genetic lesions that combine with MNA influence downstream gene expression and signaling events. Therefore, the studies completed here are reviewed in the context of the synergistic partner, Aridla, identified through this body of work, and the resulting cellular events.

Furthermore, following from recent studies in NBL with $M Y C N$ amplification, one opportunity to understand how the oncogenic action of $M Y C N$ is actualized is through the identification and study of deregulated enhancer modifying genes (Boeva et al., 2017; Decaesteker et al., 2018; Durbin et al., 2018; van Groningen et al., 2017; L. Wang et al., 2019). This approach may provide a point of connection between the amplification of $M Y C N$ and its oncogenic behavior through synergy with epigenetic regulators. In summary, $M Y C N$ is a key regulator of many genes. Targeting the downstream processes associated with $M Y C N$ driven oncogenesis is an opportunity to treat $M Y C N$-driven NBL (Boeva et al., 2017; Garrett M. Brodeur et al., 2014; Rickman et al., 2018; Zeid et al., 2018). To this point, identifying the collaborative initiating events, and the resulting gene expression signatures will be vital to identifying new therapeutic targets.

\section{$A R I D 1 A$ and $M Y C$ family members}

Our system provides a unique opportunity to modify primary NCCs step by step and to functionally test the connection between genetic lesions and the transformative events they confer. To this end, in Chapter 4, we explored how Mycn OE affected cell 
death in primary cells and tumors and asked if there was a connection between Mycn overexpression and Arid la regulation. Furthermore, we evaluated whether tumors and TDCLs were competent in their DNA damage response. We did not identify consistent differences in any attributes examined in this set of experiments.

Our studies in Chapter four began with protein expression analysis of Arid1a and MYCN in a panel NBL cell lines. Although sequencing studies have confirmed ARID1A deletions in the $1 \mathrm{p} 36 \Delta$ samples, we observed that MNA samples had higher than expected Arid1a expression relative to controls (Figure 4-1A). Considering the synergy observed in tumors, we thought this finding may indicate a MYCN-regulated increase in ARID1A expression. To further test this idea, we evaluated Mycn and Aridla mRNA expression in heterozygous primary untransformed NCCs. We observed two results. First, titration of Mycn did produce a small shift in Arid1a expression (Figure 4-1D). Second, Arid1 a was expressed at levels between 65 and 85 percent relative expression. If this result holds under more rigorous analysis, then it is possible that the thresholds set during mRNA analysis to screen for potential TSGs would cause false negatives. One hallmark of cancer is escape from apoptotic cell death (D Hanahan \& Weinberg, 2000; Douglas Hanahan \& Weinberg, 2011). Next, we considered that Mycn OE may induce apoptotic pressure on WT1 samples, resulting in an escape from apoptotic death when Arid1a is heterozygously lost. To test this possibility, we used flow analysis of Annexin$\mathrm{V}$ to establish apoptotic cell fractions in primary cells treated with increasing amounts of Mycn (Figure 4-2). We expected HET samples to demonstrate a decrease in cell death relative to WT1 controls and that KO would demonstrate no difference or more sensitivity to $M y c n$-induced apoptosis. However, no significant differences were observed in the MOIs tested (MOI 0-3) (Figure 4-2A and B).

We considered that this result may be a consequence of the time points measured (72hrs). If Aridla loss resulted in a hypermutator phenotype, we expected that it might be identified in end point tumors. Next, we evaluated $\gamma \mathrm{H} 2 \mathrm{ax}$ and cleaved caspase 3 levels in end point tumors (Figure 4-3). No statistically significant differences were observed in HET tumors relative to controls (Figure 4-3A and B), however KO tumors displayed a statistically significant increase in Caspase-3 (Figure 4-3C and D). Considering that Aridla has been identified as a regulator of the DNA damage response, is mutually exclusive of $\mathrm{p} 53$, and has been linked to high rates of mutation, we were surprised that these traits were not evident in our study.

Furthermore, in our model, no significant differences in resistance, sensitivity, or ATR signaling of DNA damage were overserved in response to treatment of TDCLs with chemotherapeutics (Figures 4-4 and 4-6). This study was limited to time points of 72hrs and under. In the apoptosis experiments using primary cells, pilot studies out to 96 hours were performed, and 72 hours was expected to capture differences. However, repeating the experiments and extending the time points may highlight potential differences. Furthermore, culturing cells in vitro using media spiked with low doses of chemotherapeutics over weeks may highlight possible differences in drug resistance across TDCLs. 
To summarize, our studies evaluated the hypothesis that Mycn OE inducesAridla expression to signal an apoptotic response that would be abrogated by Aridla deletions. This hypothesis was proven false to the extent tested. However, the increase in Aridla protein and mRNA suggests a feedback loop between ARIDIA and MYCN that supports the concept of co-regulation. Closer study of this system may uncover co-regulated genes and the mechanisms that control them.

\section{N-MYC and TWIST1 co-regulation of enhancers}

Recently, one study determined how different $M Y C N$ expression levels regulated MYCN positioning at promoters and enhancers in NBL (Zeid et al., 2018). They identified that at promoters with canonical E-box sites, MYCN binding overlapped with MYC binding signatures, however MYCN preferentially invaded enhancers that were established critical lineage factors of the developing SNS (Boeva et al., 2017; Zeid et al., 2018). Furthermore, MYCN amplified samples had a symbiotic relationship with TWIST1, a pioneering factor of stemness and migration (Zeid et al., 2018). High levels of TWIST1 cooperated with MYCN to facilitate binding at distal enhancers, suggesting that MYCN binding at non-canonical sites is regulated by cell type-dependent proteins. Although evident that TWIST1 expression correlated with MYCN binding at these critical enhancer sites, the mechanism was not indicated. This paper applied a known approach in a unique way. MYCN was studied by leveraging Chip-seq consistently throughout the article. This approach used the binding of promoters and enhancers, a key function of this TF, as a readout for $M Y C N$ activity following the manipulation of its expression. Importantly, they defined one new possibility for how MYCN exerts its oncogenic function, through cooperation with TWIST1 (Zeid et al., 2018).

TWIST1 is a bHLH transcription factor that can form either homo or heterodimers before binding to E-box sites in promoters of its target genes (Castanon \& Baylies, 2002; Merindol et al., 2014). Twist1 was identified in cranial neural crest cells as a key regulator of migration and stemness (Soldatov et al., 2019). Furthermore, Twist1 expression has been shown to regulate trans-differentiation of neuronal and mesenchymal cell identity in cardiac NCCs (Vincentz et al., 2013). In trunk NCCs, TWIST1 expression is regulated, at least in part, by PBAF, which was demonstrated in human NCCs (Bajpai et al., 2010). In NBL, TWIST1 expression is correlated to MNA tumors, and poor overall patient survival (Selmi et al., 2015; Valsesia-Wittmann et al., 2004; Zeid et al., 2018). Two studies have suggested that N-MYC regulates TWIST1 expression, and one suggested that MYC regulated TWIST1 expression, and a recent article determined that MYC and TWIST1 cooperate to induce metastasis (Valsesia-Wittmann et al., 2004; Selmi, de Saint-Jean, Jallas, Garin, Hogarty, Bernard, et al., 2015; Zeid et al., 2018). Although N-MYC was shown to bind the TWIST1 promoter, experiments testing the effects of MYCN modulation of TWIST1 expression have been inconsistent, suggesting additional regulators of TWIST1 may contribute to its high expression levels in MNA cases (Selmi et al., 2015; Valsesia-Wittmann et al., 2004; Zeid et al., 2018). 
Following from this more recent picture of $M Y C N$ amplification, one opportunity to understand how the oncogenic action of $M Y C N$ is actualized is through the identification and study of deregulated enhancer modifying genes. This approach may provide a point of connection between the amplification of $M Y C N$ and its oncogenic behavior through synergy with epigenetic regulators. In summary, $M Y C N$ is widely expressed and is a key regulator of many genes. Targeting the downstream processes associated with $M Y C N$ driven oncogenesis is an excellent opportunity to treat $M Y C N$ driven NBL (Garrett M. Brodeur et al., 2014). To this point, identifying the collaborative initiating events, and the resulting gene expression signatures will be vital to identifying new therapeutic targets.

\section{Potential synergies of ARID1A and N-MYC}

Does ARID1A regulate TWSIT1 expression? Our data demonstrates that Arid1a deletion causes an increase in TWIST1 expression in HET and KO tumors (Figure 3-10A). TWSIT1 is a master regulator of the normal NCC EMT process, and is an initiating mutation in metastasis (Yang et al., 2004). Furthermore, the migration rates of HET and KO TDCLs correlate to TWSIT1 expression levels in tumors. NCCs undergo EMT as they migrate away from the neural tube and differentiate into the SNS (Jiang et al., 2013; Kuo \& Erickson, 2010; Soldatov et al., 2019). Interestingly, N-MYC and TWSIT1 expression have been correlated in NBL by two groups, although the exact mechanism of regulation has not been published (Selmi et al., 2015; Zeid et al., 2018).

We demonstrated that Aridla heterozygous loss impaired differentiation and induced an EMT gene expression signature (Figures 3-6 through 3-10). Taken together, our studies support the role of Aridla in EMT described in the current literature and contributes additional details to the context dependent effects of Aridla in cancer initiation (Han et al., 2019; Sun, Chuang, et al., 2017; Sun, Wang, et al., 2017; W. Wang et al., 2019). EMT is a highly regulated process that may become deregulated and initiate metastasis (Yang et al., 2004). Our study supports the concept of a link between these behaviors and cell maturity. Although suspected, failure to exit EMT has not previously been linked to cancer initiation in NBL (Etchevers et al., 2019; Furlan et al., 2017; Soldatov et al., 2019).

\section{The $A R I D 1 A$ tumor suppressor gene}

Identification of a causal role for Aridla in our model may elucidate areas for continued studies in HR NBL and may also influence how the SWI/SNF field studies sub-unit loss. ARID1A protein function cannot be determined solely based on protein expression, and our work suggests that genetic screens, and protein expression will need to be complimented with immunoprecipitation (IP) analysis of the SWI/SNF complexes (Figure 4-1). For example, preliminary evaluation ARID1A protein levels in MNA NBL cases, and in response to Mycn titration suggest that ARID1A expression may be upregulated even when one copy of the gene is lost. The use of IPs to pull down the 
SWI/SNF complex following Aridla deletion or ARIDIA mutation, can determine if the complex composition changes. For example, does ARID1B or ARID2 become the predominant ARID subunit in the new complex (Bajpai et al., 2010; Helming et al., 2014)? Furthermore, Zied et al., performed an analysis of MNA by not only evaluating protein expression, but by using ChIP-seq binding patterns to connect expression levels to activity (Zeid et al., 2018). Adapting their approach to a chromatin remodeling complex would be a useful way to fully resolve the functionality and outcomes of chromatin remodeler mutations, such as Arid1a loss in the SWI/SNF complex. As Zeid et al. demonstrated, ChIP experiments can evaluate SWI/SNF targets when ARID1A is absent (Zeid et al., 2018). Combining these approaches will likely provide more clarity in studies aiming to represent how SWI/SNF functions in cancers with ARID1A mutations.

Our data opens the possibility that some epigenetic TSGs may operate under the goldilocks principle, rather than the framework posited by the two hit hypothesis or current definitions of haploinsufficiency (Berger \& Pandolfi, 2011; Knudson, 1971; D. S. Park et al., 2019). Dose dependent TSGs, like P53 demonstrate a partial phenotype when 1 gene copy is lost, but the phenotype is further exacerbated when both gene copies are lost, thus demonstrating that P53 haploinsufficiency is sufficient for tumor transformation (Berger \& Pandolfi, 2011). Another type of haploinsufficiency is demonstrated by the PTEN TSG (Berger \& Pandolfi, 2011). Haploinsufficient PTEN loss is sufficient to induce cancer, however full ablation induced senescence (Berger \& Pandolfi, 2011; Chen et al., 2005). This is termed obligate haploinsufficiency (Berger \& Pandolfi, 2011).

For example, tumor latency differences were insignificant between $\mathrm{KO}$ compared to WT2 tumor kinetics (Figures 3-1 through 3-3). Although HET tumors demonstrated reduced latency. Further TSG studies across models and cell types will be needed to confirm the effects demonstrated by Aridla dose dependent loss. For example, in embryoid bodies evaluated in this mouse model, one study reported differences between Aridl $a$ heterozygous and knockout deletions (Gao et al., 2008). In adult cancer studies, Aridla heterozygous deletions have demonstrated a tumorigenic advantage during tumor outgrowth, but not initiation in MYC-driven mouse models of liver cancer (Sun, Chuang, et al., 2017; Sun, Wang, et al., 2017). Our data suggests that in the context of Mycndriven NBL, heterozygous Aridla deletion provide the most advantage during initiation, indicating haploinsufficiency. However, rather than $\mathrm{KO}$ inducing senescence, we found that $\mathrm{KO}$ reduced tumor initiation kinetics to that of WT2 tumors. We consider this to demonstrate the goldilocks principle, where there is a critical gene dose for transformation (Amin, Rajan, Groysman, Pongtornpipat, \& schatz, 2015; D. S. Park et al., 2019). Our study supports conclusions by by Sun et al. that suggest ARIDIA is a dose and context dependent TSG (Mathur, 2018; Sun, Wang, et al., 2017; J. N. Wu \& Roberts, 2013). It is possible that a new category of TSGs may be uncovered as more epigenetic TSGs are evaluated, and that phenotypes may be tissue and cancer type specific (Sun, Wang, et al., 2017). However, further causal examination in mouse models of cancer will be necessary to confirm our report. 
Aridla loss has been implicated in adult cancer as an essential mediator of wound healing, and initiator of metastatic spread (Sun, Chuang, et al., 2017; Sun, Wang, et al., 2017). Taken together, multiple studies and our results suggest that EMT is involved (Han et al., 2019; Sun, Chuang, et al., 2017; Sun, Wang, et al., 2017; S. C. Wang et al., 2019). TWIST1 is a master regulator of EMT, and its overexpression has been causally determined to increase metastatic spread of mammary tumors (Yang et al., 2004). EMT induces a quasi-mesenchymal state, and is thought to induce cancer stem cells, although a direct mechanism describing how EMT induces stemness has not been established (Dongre \& Weinberg, 2019). One area of metastatic spread that is lacking primary literature is an understanding of the traits that confer an advantage to migrating cells allowing them to colonize new tissue sites (Dongre \& Weinberg, 2019). One possibility is that stem-like traits are a feature of the original stem cell, rather than the EMT TFs, which might explain some of the overlap between EMT and metastasis seen in various cancer types (Etchevers et al., 2019).

While tumors were locally invasive, metastatic disease was not observed in our sub-cutaneous mouse model. However, our molecular analysis suggested, and functional analysis confirmed that tumors with Aridla deletions had higher rates of migration than WT controls (Figure 3-9B and C). Furthermore, one signaling node of genes concordantly up-regulated in HET and $\mathrm{KO}$ was defined by multiple metalloproteases (Figure 3-8A). Also considering the gene signatures identified in our study, we interpreted these studies to collectively indicate an increase in metastatic potential of Aridla mutant NCCs.

Despite the wide relevance of Aridla heterozygous mutation rates across adult and pediatric cancers, to date, few cancer studies have directly tested the ability of Aridla heterozygous deletions to drive cancer in mouse models, and this work was performed in adult cancers (Mathur et al., 2017; Sun, Wang, et al., 2017; S. C. Wang et al., 2019). The most prevalent types of Aridla cancer studies are performed in cancer cell lines with siRNA mediated knockdown, or in models of Aridla homozygous deletions (Guan et al., 2011; Lord et al., 2016; Shen et al., 2018). Specifically, in one Aridla deleted mouse model of colon cancer, Aridla was identified as a context-dependent TSG that was found to control tumor metastasis (Sun, Wang, et al., 2017). Interestingly, in this adult cancer model, Aridla deletion was not implicated in tumor initiation of liver cancer (Sun, Wang, et al., 2017). Our experimental findings of the Aridla dose dependent regulation of motility fit with this previously established liver cancer model. However, in addition to regulating migration, multiple biologically independent mouse studies confirmed that Aridla deletion synergized with $M Y C N$ amplification in NBL initiation. The difference between the two Aridla mouse models is intriguing. One concept that may be useful in framing the results from this pediatric study relative to the adult cancer study, is the role of epigenetic mutations in pediatric disease.

Pediatric cancers were recently shown to have a high incidence of epigenetic mutations relative to adult cancer (Filbin \& Monje, n.d.; Flavahan et al., 2017; Gröbner et al., 2018). Pediatric cancers can be considered a disease of development, and epigenetic factors direct the biological systems of development. Alterations in cell fate contribute to 
tumorigenesis (Vogelstein, Papadopoulos, \& Velculescu, 2013). The high incidence of epigenetic mutations in pediatric cancers suggest that mutations in epigenetic regulators during development influence cell fate. Outside of speculating why one mutation, such as ARIDIA los of function mutations, may disproportionally affect one cell type over another, there is one conclusion that can be drawn when considering the role of ARIDIA in disease. Functional testing of genetic events via context specific disease models is extremely important for identifying cancer drivers and the resulting downstream consequences (Berger \& Pandolfi, 2011; Garrett M. Brodeur et al., 2014; D. T. W. Jones et al., 2019; Vogelstein et al., 2013).

\section{Cell Identity}

In Chapter 3, our work identified a gene signature that correlates to mesenchymal gene signatures identified by two other studies (Soldatov et al., 2019; Zeid et al., 2018). We interpret this phenotype to be linked to cell identity, which is plastic early during development, but becomes restricted as differentiation progresses (Flavahan et al., 2017). This is consistent with large pan-pediatric cancer studies that discovered mutations in epigenetic regulators was the top alteration consistently identified across all pediatric cancers (Gröbner et al., 2018; Ma et al., 2018). In a recent pan-cancer study, pediatric cancers were reported to have $45 \%$ overlap of the driver genes mutated in adult cancer (Ma et al., 2018). Currently, Aridla has been causally studied in developmental, and adult cancer mouse models (Chandler \& Magnuson, 2016; Gao et al., 2008; Han et al., 2019; Mathur et al., 2017; Sun, Chuang, et al., 2017; Sun, Wang, et al., 2017; S. C. Wang et al., 2019). Our model combines aspects from adult cancer and developmental studies and addressed the role of Aridla as a driver mutation in trunk NCC transformation into the pediatric cancer NBL. Although we did not establish a direct molecular mechanism, our findings in this novel mouse model of NBL support the hypothesis that Aridla is a haploinsufficient TSG that synergizes with MNA to disrupt lineage development programs, resulting in increased oncogenesis due to altered cell identity.

\section{Cancer initiating stem cells}

The cancer stem cell model is characterized by four drivers of disease, cancer initiation, intratumor heterogeneity, metastatic disease and post-therapy relapse (Meacham \& Morrison, 2013). These key tumor traits are considered consequences of cancer driven by a tumorigenic stem cell population (Meacham \& Morrison, 2013). HR NBL tumors as a group fit these criteria, and we interpret this to suggest that the genes significantly contributing to disease may contribute to these phenotypes. Therefore, the development of mouse models that faithfully exhibit the genotypes and phenotypes of NBL are necessary to testing fundamental cancer biology questions.

In our model, we tested the hypothesis, does Aridla loss contribute to NCC transformation through a block in differentiation? Therefore, we focused on the role of Aridla as a loss of function initiator of Mycn-driven NBL. Consistent with this model, we 
did determine that Aridla heterozygous loss was causal in initiating Mycn-driven NBL. However, through characterizing our model, we identified signs of intratumoral heterogeneity in HET and KO tumors by pathological analysis. Although not the primary focus of this study, these results support our findings that our model faithfully recapitulates traits of NBL and suggest a rationale for further studies evaluating heterogeneity in this model.

Tumor model characterization revealed up-regulated migration and metastatic signaling nodes We evaluated the evidence of metastatic disease in our model in vivo, in vitro, and with RNA-sequencing to better identify the potential metastatic effects of HET and $\mathrm{KO}$ cell populations. Furthermore, tissue invasion and metastatic disease is one of the six original hallmarks of cancer and is a key trait of the cancer stem cell model (Douglas Hanahan \& Weinberg, 2011; Meacham \& Morrison, 2013; Yang et al., 2004). One interesting study of Arid1a in a MYC-driven model of liver cancer found that one and two-copy loss of Aridla did not initiate disease, but rather initiated metastasis when conditionally ablated in pre-established tumors (Sun, Wang, et al., 2017). We evaluated full body histology of mice hosting tumors with long latency. These experiments did not identify metastatic colonization at distant sites however, allografts were locally invasive. Boyden chamber migration assays testing migration traits of TDLCS indicated an increase in cell motility in HET and KO populations (Figure 3-9B and $\mathbf{C}$ ). Additional results from STRING analysis of RNA-seq data identified two signaling nodes upregulated in both HET and $\mathrm{KO}$ tumors that are related to metastatic potential. These included a Twitst1 EMT signaling node, and an Adamts metalloproteinase signaling node (Figures 3-8 and 3-9A). In a breast cancer mouse model, Twist1 was causally studied and found to be an essential master-regulator of EMT and metastasis initiation (Yang et al., 2004). Adamts genes are associated with developmental and metastatic cellular programs although causal evaluation is limited (Kelwick, Desanlis, Wheeler, \& Edwards, 2015). Taken together, our results suggest that Arid1a HET and KO deletions are insufficient to induce all steps of metastasis but do increase metastatic potential.

In chapter 4, we tested TDCLs for signs of resistance or sensitivity to chemotherapeutics (Figures 4-4 and 4-5). However, we did not identify differences in HET or KO TDCLs compared to controls that would indicate a clinically significant resistance to etoposide or topotecan. Potential future studies will be discussed in the future directions section of this discussion.

\section{Cell identity master regulators}

Heterozygous Aridla deletion correlated with an immature, mesenchymal cell state (Figures 3-6, 3-7 and 3-9 through 3-12). One theme consistent throughout Aridla developmental and cancer studies, is the ability of Arid1 $a$ to regulate EMT-related phenotypes (Han et al., 2019; Sun, Wang, et al., 2017; S. C. Wang et al., 2019). EMT is both a transition state during development and an initiation step of metastasis. Failed NCC differentiation leading to inappropriate SNS patterning is considered a possible explanation for NBL initiation (Jiang et al., 2013). Given the high prevalence of 
metastatic disease in HR NBL, it is possible that transformation of highly migratory progenitors contributes to this phenotype. This fits with the suggested role of Aridla in oncogenesis, which suggests that when Aridla loss, the results in de-regulated cell commitment to differentiation or de-differentiated cells (Gao et al., 2008; Han et al., 2019; Mathur, 2018; Sun, Chuang, et al., 2017). This is supported by findings in multiple stages of development including liver, pancreatic, colorectal, and hematopoietic studies (Han et al., 2019; Mathur et al., 2017; Sun, Chuang, et al., 2017; Sun, Wang, et al., 2017; S. C. Wang et al., 2019). To evaluate the overall identity of the cells comprising the tumors in our study, we compared our RNA-seq tumor gene expression data to the NCC expression profiles recently posited by (Soldatov et al., 2019). Soldatov et al. mapped NCC maturation during development using single cell RNA sequencing to focus on the decision points of multipotent NCCs as they commit to cell fates of the SNS (Soldatov et al., 2019). Their work describes the process of cell fate decisions as a process of coactivation, competition, and eventual up-regulation of one TF over a competing TF resulting in commitment to one cell identity (Soldatov et al., 2019). Lineage tracing, causal studies, and RNA-sequencing approaches resulted in lists of the TFs determined to be the master regulators during each step of trunk NCC maturation (Soldatov et al., 2019). This comparison enabled us to connect tumor group genotypes and the expression profiles that are associated with cell identities of the developing SNS.

Cell identity studies of multipotent SNS progenitor cells have recently identified key transcription factor programs that regulate NCC cell fate (Flavahan et al., 2017; Soldatov et al., 2019). The differential genes identified in our tumors were mapped against an expression profile that corresponds to NCC fates. Interestingly, HET tumor samples mapped to a mesenchymal fate signature (Figure 3-9E), (Figure 3-10A). KO tumor sample fate mapping revealed a signature defined by stem-initiating genes and genes associated with sensory ganglia (Figures 3-10, 3-11B through F). WT1 and WT2 tumor gene signatures most closely mapped to a glial SNS signatures (Figures

3-10, 3-11A through $\mathbf{E}$ ). Due to the nature of our analysis, this RNA-seq gene signature represented a population of tumor cells. Histological analysis supported the RNA-seq data and suggested that tumors are comprised of more than one cell population (Figure 3-5B). However single-cell sequencing studies, or cell sorting studies would be necessary to better define the different cell populations and tumor compositions. In summary, our tumor analysis data supports the idea that the tumors analyzed are heterogeneous but are enriched with cells from one fate. This concept may apply to all tumor groups in this study but is particularly supported by our findings in the KO tumor group. These tumors demonstrated significant upregulation of master regulators from mesenchymal and premigratory cell profiles but mapped overall to a sensory cell profile. This work suggests that the NCC master regulator gene list provided by Soldatov et al. is a useful framework for interpreting our study. However, our RNA-seq data includes cell types represented throughout the tumor, and furthermore, our data is from transformed NCCs. It is unsurprising that fate mapping revealed an overlap of multiple gene signatures found in non-transformed $\mathrm{NC}$ progenitors. Therefore, we wanted to further investigate and group the differentially regulated genes in this model, to pull out interesting master regulator candidates in this system. 
We identified gene signatures in our tumors that were consistent with the gene expression NCC profiles developed by multiple single-cell sequencing studies (Figure 3-10A) (Furlan et al., 2017; Soldatov et al., 2019). These studies provide extensive modeling and analysis of cell-fate tracing during adrenal gland development and trunk NCC maturation, respectively (Furlan et al., 2017; Soldatov et al., 2019). We evaluated the differentially expressed genes (DEGs) in three comparison groups, HET versus WT1, $\mathrm{KO}$ versus WT2, and HET versus KO (Figure 3-11). Here, we graphically represented differences between paired tumor sample groups. Notably, the glial master regulators Plp1 and Zpf488 were upregulated in both WT1 and WT2 relative to their counterparts Aridla modified (Figure 3-11D and E). Furlan, et al. and Soldatov et al. described the glial lineage as the cell population that gives rise to Schwann cell precursors (SCPs) (Furlan et al., 2017; Soldatov et al., 2019). SCPs have been proposed as the cell type that develops into the adrenal medulla during normal murine development (Furlan et al., 2017). DEG analysis of HET tumors indicated a statistically significant increase in the sensory lineage master regulator, Eya2, and the mesenchymal fate specifier,

Twist 1(Figure 3-11A and D) (Soldatov et al., 2019). KO tumors demonstrated significant increases in Zic3, a neural plate boarded specifier, Dlx5 a regulator of delamination, Eya2 and Twist1(Figure 3-11B and E) (Soldatov et al., 2019). Eya2 and Twist1 were concordantly upregulated in HET and KO tumor samples (Figure 3-11D and E). Prrx1 was significantly upregulated in HET tumors relative to $\mathrm{KO}$, and Zic3 was significantly upregulated in KO tumor samples relative to HET tumor samples (Figure 3-11F).

HET samples demonstrated a partial block in differentiation characterized by an increase in Prrx1, a master regulator of mesenchymal identity. Twist1 was upregulated in both HET and KO tumors relative to controls. Electroporation of Twist1 to induce its overexpression in chick embryos was sufficient to re-route trunk $\mathrm{NC}$ migration, and induce a mesenchymal identity (Soldatov et al., 2019). Additionally, Twist1 was shown to up-regulate Prrx1, a master regulator of mesenchymal identity at the autonomic/mesenchymal decision point (Soldatov et al., 2019). However, KO tumors also demonstrated an increase of the sensory glia inducer the neural plate boarder specifier, Zic3, that was not up regulated in HET tumors. We interpret this suggest a more immature gene signature in $\mathrm{KO}$ relative to HET tumors that is supported by the significant block in differentiation identified by IHC (Figure 3-6D through F), neuronal differentiation IF studies (Figure 3-6H and I), and a significant Wong embryonic stem cell core signature identified by GSEA (Figure 3-9D) (Wong et al., 2008). We found it interesting that $\mathrm{KO}$ did not confer a tumorigenic advantage to NCCs relative to controls (Figures 3-1F through I, 3-2D and 3-3C) and consider the data to collectively suggest a window of transformation during NCC progenitor differentiation that left HET NCCs vulnerable to $M Y C N$-driven transformation relative to other NCC populations. In the context of the mesenchymal gene signature identified in HET samples, Twist1 was shown to increase the oncogenic effects of MYCN (Zeid et al., 2018). Prrx1 has been identified as a gene of interest that is upregulated in NBL patients at post-therapy relapse (van Groningen et al., 2017). Therefore, our data supports the concepts posited by two studies suggesting a TWIST11 collaboration with MYCN, and our study contributes the idea that other TFs regulating mesenchymal cell identity, like Prrx1, may synergize with the oncogenic function of MYCN (van Groningen et al., 2017; Zeid et al., 2018). 


\section{Arid1a heterozygous tumors model human mesenchymal NBL}

The previous data set used as a framework to interpret our gene expression results came from a study tracing non-transformed multipotent progenitor cells (Soldatov et al., 2019)s. Following from that study and considering the Aridla haploinsufficient genotype that synergized with Mycn OE in NCCs, we decided to test whether HET tumors recapitulated gene expression signatures identified in HR NBL patients. Therefore, the second dataset we used as a rubric for our model was a sequencing analysis of NBL patient samples (van Groningen et al., 2017). Evaluation of our tumors against the signature identified by van Groningen, et al supported the concept that our tumors developed a statistically significant mesenchymal gene expression signature that was concordant with human HR NBL by GSEA (Figure 3-12) (van Groningen et al., 2017). The premise of their study was to identify possible gene signatures that would help characterize the cell types comprising HR NBL tumors. Overall their project evaluated the stem cell heterogeneity in HR NBL tumors. Using the stem cell marker CD133 to sort NBL patient tumors into CD133+ and CD133- populations, and CD133+ populations generated a signature that was consistent with a mesenchymal phenotype and included the EMT genes FN1, VIM, SNAI2, and PRRX1 (van Groningen et al., 2017). In contrast, CD133- cells expressed gene signatures consistent with established adrenergic lineage markers of NBL, like PHOX2A/B, GATA2/3, and DBH which the authors termed ADRN (adrenergic) (van Groningen et al., 2017). CD133+ cells would be expected generate tumors that demonstrate aggressive traits like metastatic disease and relapse after therapy (Meacham \& Morrison, 2013). The CD133- tumors would be expected to lack tumorigenic ability or have greatly reduced tumor initiation ability (Meacham \& Morrison, 2013). Interestingly, van Groningen et al. found that both CD133+ and CD133- cells could form tumors in vitro, which may suggest that tumors are composed of cells with a flat hierarchy of cancer initiating stem cells. Interestingly, both populations were tumorigenic, however, the CD133+ cells demonstrated more aggressivity, such as in vitro resistance to chemotherapy, and they mapped more closely to NCC progenitor cells, suggesting immaturity relative to the adrenergic counterparts, which is consistent with a study by Boeva et al. (Boeva et al., 2017; van Groningen et al., 2017). To establish the master regulators of the MES phenotype, the authors enforced PRRX1 expression in the ADRN cells, which induced SNAI2 and was sufficient to re-program ADRN into a mesenchymal state (van Groningen et al., 2017). This experiment confirmed that these two cell populations were from the same lineage, and led authors to interpret that NBL cells were able to transdifferentiate, which was also proposed in another study (Boeva et al., 2017; van Groningen et al., 2017). PRRX1 positive cells were present in a small population of cells in pre-treatment patient samples but was enriched post-therapy in the two patients evaluated (van Groningen et al., 2017). This indicates a correlation between PRRX1 and NBL resistance to chemotherapy. In summary, this publication supports the idea that cell identity is regulated by master transcription factors. The authors proposed that the cell MES and ADRN cell identities were defined by TFs that form feed-forward, or core regulatory circuits at super-enhancers resulting in alterations of cell identity and tumorigenic properties (van Groningen et al., 2017). This idea is consistent with other 
recent NBL literature (Boeva et al., 2017; Decaesteker et al., 2018; Durbin et al., 2018; L. Wang et al., 2019; Zeid et al., 2018). Their conclusions provide a helpful framework that can be used to interpret our findings and consider future studies to validate TFs we identified.

Furthermore, gene expression and histological analysis support the idea that our model is consistent with altered SNS differentiation causing HET tumors to sustain a mesenchymal phenotype that is consistent with HR NBL patient tumors (Soldatov et al., 2019; van Groningen et al., 2017). Further analysis indicated that HET tumors are blocked in a state that significantly overlaps with the patient-derived MES signature indicative of HR NBL while the remaining tumor groups display a gene signature with statistically significant overlap with the ADRN signature identified in patient samples (Figure 3-10C) (van Groningen et al., 2017). Therefore, our model may be a useful tool for future testing of fundamental mechanistic questions, as high-throughput drug screening and biomarker development.

\section{Tying Together $M Y C N, A R I D 1 A$ and Cell Identity}

Pan-cancer studies of pediatric cancer identified the top significantly mutated genes to be epigenetic regulators (Gröbner et al., 2018; Ma et al., 2018). Modeling Aridl $a$ as an initiating event allowed close monitoring and capture of the downstream effects of Aridla alterations. ARIDIA literature is founded on powerful genetic and expression screens across many tumor types, and these have correlated the effects of Aridla mutations to haploinsufficient and initiating events in a variety of cancers (Gröbner et al., 2018; Kadoch et al., 2013; Tomlinson, Lambros, Roylance, \& CletonJansen, 2002; J. N. Wu \& Roberts, 2013). Altogether, these studies have demonstrated that Aridla is a context dependent TSG. Furthermore, our findings expand Aridla mouse model work into the pediatric cancer field and contribute causal evidence of an epigenetic loss of function mutation driving a HR pediatric cancer.

\section{A working model connecting $A R I D I A$ loss, $M Y C N$ enhancer invasion and TF circuits}

This dissertation has focused on the interplay of an oncogene and TSG during NBL initiation. Our studies support the hypothesis that MNA and heterozygous ARIDIA synergize to disrupt normal NCC differentiation during a window of transformation. To

summarize, we now describe a possible mechanism behind this synergy and its effects on cell identity. TWIST1 is a helpful example in thinking about this mechanism. $M Y C N$ has been shown to bind to the promoter of TWIST1 in NBL (Selmi et al., 2015; ValsesiaWittmann et al., 2004; Zeid et al., 2018). However experiments performed in these three studies did not demonstrate an increase in TWIST1 protein levels following increased MYCN expression (Selmi et al., 2015; Valsesia-Wittmann et al., 2004; Zeid et al., 2018). Taken together, it is possible that MYCN does not directly regulate Twist1 expression. It is clear that MYCN and TWIST1 synergize to bind enhancers (Zeid et al., 2018). This 
leaves us with the question, if not MYCN, then what causes TWIST1 up-regulation in MNA NBL? Our studies identified that Twist1 mRNA expression was significantly increased in tumor samples when Aridla expression decreased $(n=8)$. This suggests that Twist 1 expression is regulated by Aridla at the mRNA level. Furthermore, the mRNA gene signature of up-regulated genes in HET samples indicates that loss of Aridla leads to an up regulation of multiple TFGs - especially TFGs that regulate EMT. TF circuits have been shown to produce feed-forward loops that bind one another's super enhancers and define cell identity (Hnisz et al., 2013; van Groningen et al., 2017; Whyte et al., 2013; Young, 2011). Considering the findings presented here, we modify our hypothesis to include a mechanism and to propose the working model that loss of function ARIDIA mutations disrupt enhancers that regulate cell identity, thus, leaving enhancer binding sites open to act as reservoirs for abundant proteins, such as MYCN (Zeid et al., 2018). Furthermore one may envision the possibility that if master regulators of stemness are deregulated and overexpressed in this environment, for example MYCN and TWIST1, these oncogenic proteins may saturate normal DNA binding sites resulting in enhancer invasion, the reprogramming of normal cell fate commitment TF circuits and consequently holding NCCs in an immature cell state that is oncogenic.

\section{Disease modeling - key features and limitations}

Our mouse model was neither spontaneous nor orthotopic. Lack of modeling in the adrenal medulla was a limitation and an advantage of our study. For example, in our sub-cutaneous model, tumors were measured on a regular basis without the administration of luciferase and use of an imaging machine (D. T. W. Jones et al., 2019). Furthermore, compared to spontaneous models, gene modifications in primary cells can be added in vitro, without the need to produce an additional mouse model (D. T. W. Jones et al., 2019). Although we did not use an orthotopic model, one insight into the origin of NBL can be gleaned from sub-cutaneous disease modeling. Taken together, our data suggests that modifying NCCs before sub-cutaneous injection faithfully models this sub-type of $M Y C N$-driven NBL.

One challenge in relating our model to the field's experimental results can be easily contextualized with the following example. In bioinformatic analysis that compared samples from our four tumor groups to the patient samples derived by van Groningen et al., we saw a significant overlap of our model's gene expression signature compared to their patient samples' gene expression signature (van Groningen et al., 2017). However, the patient samples' genetic characterization at the time of analysis was unpublished, making this comparison valuable, but open to the following questions: Do the patient samples analyzed by van Groningen, et al. have $M Y C N$ amplification and/or 1p36 LOH? Additionally, during CD133 separation by cell sorting, are there any chromosomal differences in the positive vs negative groups that may further support a link of chromosomal mutations to cell identity? The ADRN/MES distinction overall is an interesting approach to grouping patient tumors based on gene expression signatures and provides a helpful rubric for the comparison and evaluation of our model. However, chromosomal information, such as amplifications and deletions in patient samples, 
remains an important refence in order to classify and compare samples across patients and experimental models. Additional consideration for referencing samples based on genotype would be an excellent way to organize and streamline the communication around experimental findings and appropriately correlate experimental results back to patient samples. Improving this step in communicating experimental findings in the NBL field would improve the speed and accuracy of interpreting experimental findings as it relates to patient samples. Our data supports the hypothesis proposed by Dr. Brodeur, that 1p36 LOH contributes to tumor aggressivity (Brodeur, Sekhon and Goldstein, 1977; Maris et al., 1995; Brodeur, 2003). As the NBL field moves toward precision medicine, communicating the exact genetic events found in patients will become increasingly important.

\section{Future Directions}

Oncogene and tumor suppressor gene disruptions are fundamental to tumorigenesis. Identifying driver mutations is critical to resolving each cancer type's unique genetic footprint and gene expression signatures. However, the gene expression cascades that act downstream of driver mutations to signal these disruptive inputs are opportunities for synthetic-lethal therapeutics. It is obvious that oncogenes and tumor suppressors that are frequently mutated together enable a proliferative advantage and tumor onset. A better understanding of the regulatory mechanisms essential to the establishing feedback loops between these driver mutations, leading to their synergy, may provide insights into the disrupted core regulatory circuits in NBL. Identifying the genes that connect mutant TSGs and oncogenes may uncover options for therapeutic targeting that would disrupt feedback and feed-forward oncogenic circuits and ultimately reduce tumor burden (Garrett M. Brodeur et al., 2014; Durbin et al., 2018; van Groningen et al., 2017; L. Wang et al., 2019; Zeid et al., 2018).

Our trunk neural crest explant model is positioned to connect developmental biology concepts to the pediatric cancer community. An additional advantage of this NCC cell model is the option to modify progenitor cells using combinations of GEMM and gene editing techniques simultaneously. For example, 17q translocations have been identified as the most frequent chromosomal abnormality in HR NBL (Garrett M. Brodeur et al., 2014; Davidoff, 2012; Pugh et al., 2013; Tolbert \& Matthay, 2018). Modeling candidate oncogenic chromosomal events in these primary cells may further pinpoint the genetic events that are causal in de-regulation of normal cell properties and transformation of NCCs into NBL.

This body of work connected epigenetic de-regulation to the oncogenic action of $M Y C N$ through a demonstrating a collaboration between Aridla loss and MYCN overexpression. Furthermore, gene expression profiling identified a set of genes that are linked to altered cell identity in other cancer models. Finally, a de-regulated cellular identity was characterized by in vitro assessment of primary cells, and in vivo tumorigenesis studies and subsequent analysis of tumor tissue and TDCLs. Following from this work, there are key experiments that could be performed to validate the 
findings discussed in this dissertation and propel this work toward clinical applications. To close this discussion, I have developed an approach of validation and pre-clinical modeling of these findings that is described below. I end this discussion with a short list of ideas that would link this body of work to outstanding NBL questions that may be addressed through additional experimentation in our model.

\section{Functional validation of the Arid1a-regulated gene signature}

First, the up-regulated Aridl $a$-dependent gene targets identified by RNAsequencing studies need to be confirmed with Aridla modulation experiments. A sleeping beauty transposase system can be used to re-introduce Aridla into TDCLs. Next, the Aridla depletion and re-introduction could be confirmed with immunoprecipitation analysis of the SWI/SNF complex, shown in Figure B-2. If it is possible to modulate Aridla's association with the complex following the re-introduction of Aridla, a qPCRbased check for Aridla-dependent modulation of the proposed Aridla targets before and after Arid1a addition would validate the findings of this work and suggest top hits.

Next, small molecule inhibitors (SMIs) could be used to target and modulate the Aridla down-stream genes that regulate EMT and differentiation. For positive controls, SMIs can be pooled 5+ (each for a different target), before assaying multiple SMIs that target the same protein. To confirm SMI efficacy, one or two down-stream targets could be validated using an ELISA, which can be purchased or built in-house.

Once Aridla-dependent targets and SMIs have been validated, high-throughput screening methods using robotics monitoring of cell viability and migration could be used to determine whether cell viability and migration are affected by inhibition of target proteins. The top 10 SMIs that affect both viability and migration would then pass into the next set of screens.

Hits from the SMI in vitro screen would need to be screened in vivo. First, a pilot experiment would need to be performed using only controls to determine the critical experimental conditions. Only two cell lines would be necessary for the pilot study- one Aridl $a^{\mathrm{WT}}+M Y C N$ OE (Aridla re-expressing if possible), and one Aridla ${ }^{\mathrm{HET}}+M Y C N$ $\mathrm{OE}$ cell line. Injecting $1.5 \mathrm{e} 6$ cells per mouse would ensure time until tumor could be accurately monitored and treated after establishment $(300 \mathrm{~mm} 3)$. To establish the controls for SMIs, one drug that failed from the first screen could serve as a negative control. DMSO should also be included as a negative SMI control. A combination of all drugs could serve as positive SMI controls (60 mice) (Table 5-1).

Following the establishment of experimental conditions, the in vitro hits could be confirmed in vivo using results from the pilot experiment in Table 5-1. In all experiments, at least one Aridla ${ }^{\mathrm{WT}}$ TDCL treated with one of the positive SMI controls and a DMSO negative control would need to be included. Injecting five mice per drug 
Table 5-1. Pilot drug study in mice.

\begin{tabular}{lccc}
\hline & \multicolumn{3}{c}{ Cell Lines } \\
\cline { 2 - 4 } Drug & Arid1a $^{\text {WT }}$ & Arid1a $^{\text {Re-WT }}$ & Arid1a $^{\text {HET }}$ \\
\hline DMSO (- ctrl) & $\mathrm{n}=5$ & $\mathrm{n}=5$ & $\mathrm{n}=5$ \\
Failed Drug (- ctrl) & $\mathrm{n}=5$ & $\mathrm{n}=5$ & $\mathrm{n}=5$ \\
Combo 1 (+ ctrl) & $\mathrm{n}=5$ & $\mathrm{n}=5$ & $\mathrm{n}=5$ \\
Combo 2 (+ ctrl) & $\mathrm{n}=5$ & $\mathrm{n}=5$ & $\mathrm{n}=5$ \\
\hline
\end{tabular}

Notes: $(-\mathrm{ctrl})=$ negative control, $(+\mathrm{ctrl})=$ positive control, Arid1 $\mathrm{a}^{\mathrm{re}-\mathrm{WT}}=$ Aridla depleted TDCL with re-expression of Aridla and Mycn OE, Arid1a ${ }^{\mathrm{HET}}=$ Aridla heterozygous TDCL with Mycn OE. 
would ensure top drug hits could be separated from negative controls (Table 5-2). To allow appropriate monitoring of the experiment, the top 10 list of SMI hits should be split in half, resulting in two experiments that include five SMIs each (68 mice for 5 SMIs). Repeating Table 5-2 a second time would complete in vivo screening of the top $10 \mathrm{SMI}$ hits (136 mice total).

Dissociated patient-derived xenografts could be used to confirm top in vivo hits in human samples. NBL xenografts would need to be tested for (proximal) $1 \mathrm{p} 36$ and $M Y C N$ amplification status first. The following xenograft genetic sub-types would be needed for experimental and control groups. Xenografts: 1x Non-MNA/1p36 LOH (negative control), 3x MNA alone (1p36 negative control), 4x MNA + 1p36 LOH (NBL sub-type of interest), 3x 1p36 LOH (experimental group to test effects of this deletion in the absence of $M Y C N$ ). These sub-groups could be supplemented with cell lines if necessary. Descriptions of genetic alterations in NBL cell lines can be found in Table A-1. Following sample validation, xenografts could be injected into their respective original host mouse strains in a pilot study. Injecting a varied number of cells per mouse would determine optimal establishment and growth conditions. Recommended doses are 1.5e6, 3e6, and 9e6 (99 mice) (Table 5-3). Once the optimal conditions are established, hits from the Aridla SMI screen in human xenografts could be validated in the xenografts (341 mice for five SMIs) (Table 5-4).

\section{Intratumoral heterogeneity}

Another avenue that would be interesting to explore in more detail, is fate mapping of the cells that initiate tumorigenesis, followed by single-cell sequencing to generate gene expression profiles that are consistent across the tumor sub-types generated in this study. This approach is not only relevant to NBL and could easily be expanded to other adult and pediatric cancer types. However, for simplicity in our model, I have generated over 100 cell lines that could be transduced with a ROSA-confetti expressing virus, and re-injected orthotopically, or sub-cutaneously, then harvested at $300 \mathrm{~mm} 3$, and cell sorted into populations for evaluation.

This experiment could be approached with a limiting dilution series that would be calculated based on the number of colors expressed by the ROSA system. This is necessary to evaluate and expand rare populations of stem-cells presumed to initiate this disease. Unfortunately, no true tumor stem cell markers have been established in NBL. Currently used markers include CD133, and CD44 (van Groningen et al., 2017). CD44 was upregulated in Ardil a HET and KO tumors according to RNA-seq analysis, and subsequently, may be a useful marker in our system. Additional projects in the Freeman lab are related to the identification of NCC markers that can be used for lineage tracing and may elucidate other tumor stem cell markers relevant to our system. 
Table 5-2. Drug study in mice.

\begin{tabular}{lcc}
\hline Drug & Aridla $^{\text {Re-WT }}$ & Arid1 $^{\text {HET }}$ \\
\hline DMSO (- ctrll) & $\mathrm{n}=3$ & $\mathrm{n}=3$ \\
Failed Drug (- ctrl) & $\mathrm{n}=3$ & $\mathrm{n}=3$ \\
Combo 1 (+ ctrl) & $\mathrm{n}=3$ & $\mathrm{n}=3$ \\
SMI 1 & $\mathrm{n}=5$ & $\mathrm{n}=5$ \\
SMI 2 & $\mathrm{n}=5$ & $\mathrm{n}=5$ \\
SMI 3 & $\mathrm{n}=5$ & $\mathrm{n}=5$ \\
SMI 4 & $\mathrm{n}=5$ & $\mathrm{n}=5$ \\
SMI 5 & $\mathrm{n}=5$ & $\mathrm{n}=5$ \\
\hline
\end{tabular}

Notes: $(-\operatorname{ctrl})=$ negative control, $(+\operatorname{ctrl})=$ positive control, Arid1 ${ }^{\text {re-WT }}=$ Aridla depleted TDCL with re-expression of Aridla and Mycn OE, Arid1a ${ }^{\mathrm{HET}}=$ Aridla heterozygous TDCL with Mycn OE.

Table 5-3. Validation of xenograft growth in mice.

\begin{tabular}{|c|c|c|c|c|c|c|c|c|c|c|c|}
\hline \multirow[b]{2}{*}{ Cell \# } & \multirow{2}{*}{$\begin{array}{c}\text { Non- } \\
\text { MNA/ } \\
1 \mathrm{p36} \\
1 \\
\end{array}$} & \multicolumn{3}{|c|}{ MNA } & \multicolumn{4}{|c|}{ MNA + 1p36 } & \multicolumn{3}{|c|}{$1 p 36 \mathrm{LOH}$} \\
\hline & & 1 & 2 & 3 & 1 & 2 & 3 & 4 & 1 & 2 & 3 \\
\hline $1.5 \times 10^{6}$ & $\mathrm{n}=3$ & $n=3$ & $\mathrm{n}=3$ & $n=3$ & $n=3$ & $n=3$ & $n=3$ & $\mathrm{n}=3$ & $n=3$ & $\mathrm{n}=3$ & $n=3$ \\
\hline $3.0 \times 10^{6}$ & $n=3$ & $n=3$ & $n=3$ & $n=3$ & $n=3$ & $n=3$ & $n=3$ & $\mathrm{n}=3$ & $n=3$ & $\mathrm{n}=3$ & $n=3$ \\
\hline $9.0 \times 10^{6}$ & $\mathrm{n}=3$ & $n=3$ & $n=3$ & $n=3$ & $n=3$ & $n=3$ & $n=3$ & $\mathrm{n}=3$ & $\mathrm{n}=3$ & $\mathrm{n}=3$ & $\mathrm{n}=3$ \\
\hline
\end{tabular}

Notes: $\mathrm{MNA}=M Y C N$ amplified, $\mathrm{LOH}=$ loss of heterozygosity 
Table 5-4. Xenograft drug study.

\begin{tabular}{|c|c|c|c|c|c|c|c|c|c|c|c|}
\hline \multirow[b]{2}{*}{ Sample \# } & \multirow{2}{*}{ 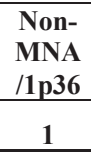 } & \multicolumn{3}{|c|}{ MNA } & \multicolumn{4}{|c|}{ MNA + 1p36 } & \multicolumn{3}{|c|}{$1 \mathrm{p} 36 \mathrm{LOH}$} \\
\hline & & 1 & 2 & 3 & 1 & 2 & 3 & 4 & 1 & 2 & 3 \\
\hline DMSO (- ctrl) & $\mathrm{n}=3$ & $\mathrm{n}=3$ & $\mathrm{n}=3$ & $\mathrm{n}=3$ & $\mathrm{n}=3$ & $n=3$ & $\mathrm{n}=3$ & $\mathrm{n}=3$ & $\mathrm{n}=3$ & $\mathrm{n}=3$ & $\mathrm{n}=3$ \\
\hline Combo $(+$ ctrl $)$ & $\mathrm{n}=3$ & $\mathrm{n}=3$ & $\mathrm{n}=3$ & $\mathrm{n}=3$ & $\mathrm{n}=3$ & $\mathrm{n}=3$ & $\mathrm{n}=3$ & $\mathrm{n}=3$ & $\mathrm{n}=3$ & $\mathrm{n}=3$ & $\mathrm{n}=3$ \\
\hline SMI 1 & $\mathrm{n}=5$ & $\mathrm{n}=5$ & $\mathrm{n}=5$ & $\mathrm{n}=5$ & $\mathrm{n}=5$ & $\mathrm{n}=5$ & $\mathrm{n}=5$ & $\mathrm{n}=5$ & $\mathrm{n}=5$ & $\mathrm{n}=5$ & $\mathrm{n}=5$ \\
\hline SMI 2 & $\mathrm{n}=5$ & $\mathrm{n}=5$ & $\mathrm{n}=5$ & $\mathrm{n}=5$ & $\mathrm{n}=5$ & $\mathrm{n}=5$ & $\mathrm{n}=5$ & $\mathrm{n}=5$ & $\mathrm{n}=5$ & $\mathrm{n}=5$ & $\mathrm{n}=5$ \\
\hline SMI 3 & $\mathrm{n}=5$ & $\mathrm{n}=5$ & $\mathrm{n}=5$ & $\mathrm{n}=5$ & $\mathrm{n}=5$ & $\mathrm{n}=5$ & $\mathrm{n}=5$ & $\mathrm{n}=5$ & $\mathrm{n}=5$ & $\mathrm{n}=5$ & $\mathrm{n}=5$ \\
\hline SMI 4 & $\mathrm{n}=5$ & $\mathrm{n}=5$ & $\mathrm{n}=5$ & $\mathrm{n}=5$ & $\mathrm{n}=5$ & $\mathrm{n}=5$ & $\mathrm{n}=5$ & $\mathrm{n}=5$ & $\mathrm{n}=5$ & $\mathrm{n}=5$ & $\mathrm{n}=5$ \\
\hline SMI 5 & $\mathrm{n}=5$ & $\mathrm{n}=5$ & $\mathrm{n}=5$ & $\mathrm{n}=5$ & $\mathrm{n}=5$ & $\mathrm{n}=5$ & $\mathrm{n}=5$ & $\mathrm{n}=5$ & $\mathrm{n}=5$ & $\mathrm{n}=5$ & $\mathrm{n}=5$ \\
\hline
\end{tabular}

Notes: $\mathrm{MNA}=M Y C N$ amplified, $\mathrm{LOH}=$ loss of heterozygosity 


\section{Modeling and testing metastatic disease}

Metastasis has been described as a multistep process that leads to dissemination of primary disease and colonization at distant sites (Dongre \& Weinberg, 2019). The study by Sun et al that found loss of Aridla was an initiator of metastasis but not tumorigenesis is intriguing. Paired with our study indicating that Aridla loss is a driver of a frequently metastatic disease, these findings warrant further exploration. For example, it would be informative to compare metastatic biopsies to primary tumors then evaluate them for Arid1a deletions. Furthermore, analysis of the expression levels of other genes, like TWIST1 and PRRX1 in metastatic colonies would directly test hypotheses posited by multiple groups (Soldatov et al., 2019; Sun, Wang, et al., 2017; van Groningen et al., 2017).

Furthermore, our NBL cancer model indicated altered EMT, but did not demonstrate an increase in metastatic disease. However, Sun et al. demonstrated a significant increase in metastatic disease following Aridla deletion (Sun, Wang, et al., 2017). The discrepancy between these results may be due to a difference in the cancer models, but there are additional interesting plausible biological explanations: 1: The $\mathrm{SWI} / \mathrm{SNF}$ complex is comprised of different members in each cell type, resulting in over 288 combinations; therefore, deletion of Aridla has different outcomes in HCC versus NBL models (Weissman \& Knudsen, 2009). 2: ARID1A loss deregulates the same set of genes (EMT factors) in all cell types, and this has a variety of effects depending on the cell type and differentiation stage, ranging from a block in differentiation in progenitor cells, to increased metastatic potential in differentiated cells. Mouse models of disease will be increasingly useful in determining the effects of $A R I D I A$ mutations across pediatric and adult cancers. We have precisely regulated Aridla dosage in the NCC cell type, and have determined that Aridla loss blocks the differentiation of NCCs. Our data supports the hypothesis that ARIDIA is a haploinsufficient 1p36 TSG that collaborates with MYCN in NBL initiation.

\section{Conclusion}

The results presented in this body of work highlight the importance of context specific disease modeling, especially in high-mortality orphan diseases such as this pediatric cancer. Not only do these findings identify a distinct set of genes with functional consequences in cancer, they also direct our focus to potential new drug targets for MNA NBL. Finally, this data functionally and molecularly supports data from studies across a variety of ARIDIA mutated cancers and expands not only the NBL field's understanding of an epigenetic regulator in pediatric cancer, but also provides higher resolution and connectivity to 25 cancer types by furthering our understanding of the most mutated member of the SWI/SNF epigenetic complex and its connection to cell identity. 


\section{LIST OF REFERENCES}

Ahmed, A. A., Zhang, L., Reddivalla, N., \& Hetherington, M. (2017, April 3).

Neuroblastoma in children: Update on clinicopathologic and genetic prognostic factors. Pediatric Hematology and Oncology, Vol. 34, pp. 165-185. https://doi.org/10.1080/08880018.2017.1330375

Alver, B. H., Kim, K. H., Lu, P., Wang, X., Manchester, H. E., Wang, W., ... Roberts, C. W. M. (2017). The SWI/SNF chromatin remodelling complex is required for maintenance of lineage specific enhancers. Nature Communications. https://doi.org/10.1038/ncomms 14648

Amin, A. D., Rajan, S. S., Groysman, M. J., Pongtornpipat, P., \& schatz, J. H. (2015). Oncogene Overdose: Too Much of a Bad Thing for Oncogene-Addicted Cancer Cells. Biomarkers in Cancer, 7s2, BIC.S29326. https://doi.org/10.4137/bic.s29326

Attiyeh, E. F., London, W. B., Mossé, Y. P., Wang, Q., Winter, C., Khazi, D., ... Maris, J. M. (2005). Chromosome 1p and 11q Deletions and Outcome in Neuroblastoma. New England Journal of Medicine, 353(21), 2243-2253. https://doi.org/10.1056/NEJMoa052399

Bajpai, R., Chen, D. A., Rada-Iglesias, A., Zhang, J., Xiong, Y., Helms, J., ... Wysocka, J. (2010). CHD7 cooperates with PBAF to control multipotent neural crest formation. Nature. https://doi.org/10.1038/nature 08733

Balmus, G., Pilger, D., Coates, J., Demir, M., Sczaniecka-Clift, M., Barros, A. C., ... Jackson, S. P. (2019). ATM orchestrates the DNA-damage response to counter toxic non-homologous end-joining at broken replication forks. Nature Communications, 10(1). https://doi.org/10.1038/s41467-018-07729-2

Berger, A. H., \& Pandolfi, P. P. (2011). Haplo-insufficiency: A driving force in cancer. Journal of Pathology. https://doi.org/10.1002/path.2800

Birkeland, A. C., Ludwig, M. L., Spector, M. E., \& Brenner, J. C. (2016). The Potential for Tumor Suppressor Gene Therapy in Head and Neck Cancer. Discovery Medicine, 21(113), 41-47. Retrieved from https://www.ncbi.nlm.nih.gov/pmc/articles/PMC4772772/

Bitler, B. G., Aird, K. M., Garipov, A., Li, H., Amatangelo, M., Kossenkov, A. V., ... Zhang, R. (2015). Synthetic lethality by targeting EZH2 methyltransferase activity in ARID1A-mutated cancers. Nature Medicine, 21(3), 231-238. https://doi.org/10.1038/nm.3799

Bitler, B. G., Fatkhutdinov, N., \& Zhang, R. (2015). Potential Therapeutic Targets in ARID1A-Mutated Cancer. Expert Opinion on Therapeutic Targets, 19(11), 14191422. https://doi.org/10.1517/14728222.2015.1062879

Bitler, B. G., Wu, S., Park, P. H., Hai, Y., Aird, K. M., Wang, Y., .. Zhang, R. (2017). ARID1A-mutated ovarian cancers depend on HDAC6 activity. Nature Cell Biology, 19(8), 962-973. https://doi.org/10.1038/ncb3582

Boeva, V., Louis-Brennetot, C., Peltier, A., Durand, S., Pierre-Eugène, C., Raynal, V., ... Janoueix-Lerosey, I. (2017). Heterogeneity of neuroblastoma cell identity defined by transcriptional circuitries. Nature Genetics, 49(9), 1408-1413.

https://doi.org/10.1038/ng.3921 
Brodeur, G M. (1995). Molecular Basis for Heterogeneity in Human Neuroblastomas. EurJ Cancer, 31A(4), 505-510. https://doi.org/10.1016/0959-8049(95)00040-p

Brodeur, G M, Sekhon, G. S., \& Goldstein, M. N. (1977). Chromosomal aberrations in human neuroblastomas. Cancer, 40(5), 2256-2263. https://doi.org/10.1002/10970142(197711)40:5<2256::aid-cncr2820400536>3.0.co;2-1

Brodeur, Garrett M. (2003, March). Neuroblastoma: Biological insights into a clinical enigma. Nature Reviews Cancer, Vol. 3, pp. 203-216. https://doi.org/10.1038/nrc1014

Brodeur, Garrett M., Iyer, R., Croucher, J. L., Zhuang, T., Higashi, M., \& Kolla, V. (2014, March). Therapeutic targets for neuroblastomas. Expert Opinion on Therapeutic Targets, Vol. 18, pp. 277-292. https://doi.org/10.1517/14728222.2014.867946

Carén, H., Fransson, S., Ejeskär, K., Kogner, P., \& Martinsson, T. (2007). Genetic and epigenetic changes in the common 1p36 deletion in neuroblastoma tumours. British Journal of Cancer, 97(10), 1416-1424. https://doi.org/10.1038/sj.bjc.6604032

Caron, H., Peter, M., Van Sluis, P., Speleman, F., De Kraker, J., Laureys, G., ... Versteeg, R. (1995). Evidence for two tumour suppressor loci on chromosomal bands 1p35-36 involved in neuroblastoma: one probably imprinted, another associated with N-myc amplification. Human Molecular Genetics, 4(4), 535-539. https://doi.org/10.1093/hmg/4.4.535

Caron, H., van Sluis, P., de Kraker, J., Bökkerink, J., Egeler, M., Laureys, G., ... Versteeg, R. (1996). Allelic Loss of Chromosome 1p as a Predictor of Unfavorable Outcome in Patients with Neuroblastoma. New England Journal of Medicine, 334(4), 225-230. https://doi.org/10.1056/NEJM199601253340404

Castanon, I., \& Baylies, M. K. (2002). A Twist in fate: Evolutionary comparison of Twist structure and function. Gene. https://doi.org/10.1016/S0378-1119(01)00893-9

Chan, W. H., Anderson, C. R., \& Gonsalvez, D. G. (2018, May 1). From proliferation to target innervation: signaling molecules that direct sympathetic nervous system development. Cell and Tissue Research, Vol. 372, pp. 171-193. https://doi.org/10.1007/s00441-017-2693-x

Chandler, R. L., Damrauer, J. S., Raab, J. R., Schisler, J. C., Wilkerson, M. D., Didion, J. P., ... Magnuson, T. (2015). Coexistent ARID1 A-PIK3CA mutations promote ovarian clear-cell tumorigenesis through pro-tumorigenic inflammatory cytokine signalling. Nature Communications, 6. https://doi.org/10.1038/ncomms7118

Chandler, R. L., \& Magnuson, T. (2016). The SWI/SNF BAF-A complex is essential for neural crest development. Developmental Biology, 411(1), 15-24. https://doi.org/10.1016/j.ydbio.2016.01.015

Chen, Z., Trotman, L. C., Shaffer, D., Lin, H. K., Dotan, Z. A., Niki, M., ... Pandolfi, P. P. (2005). Crucial role of p53-dependent cellular senescence in suppression of Ptendeficient tumorigenesis. Nature, 436(7051), 725-730.

https://doi.org/10.1038/nature03918

Cohn, S. L., Pearson, A. D. J., London, W. B., Monclair, T., Ambros, P. F., Brodeur, G. M., ... Matthay, K. K. (2009). The International Neuroblastoma Risk Group (INRG) classification system: An INRG task force report. Journal of Clinical Oncology, 27(2), 289-297. https://doi.org/10.1200/JCO.2008.16.6785 
Coughlan, D., Gianferante, M., Lynch, C. F., Stevens, J. L., \& Harlan, L. C. (2017). Treatment and survival of childhood neuroblastoma: Evidence from a populationbased study in the United States. Pediatric Hematology and Oncology, 34(5), 320 330. https://doi.org/10.1080/08880018.2017.1373315

Dallas, P. B., Pacchione, S., Wilsker, D., Bowrin, V., Kobayashi, R., \& Moran, E. (2000). The Human SWI-SNF Complex Protein p270 Is an ARID Family Member with Non-Sequence-Specific DNA Binding Activity. Molecular and Cellular Biology, 20(9), 3137-3146. https://doi.org/10.1128/mcb.20.9.3137-3146.2000

Dang, C. V. (2012, March 30). MYC on the path to cancer. Cell, Vol. 149, pp. 22-35. https://doi.org/10.1016/j.cell.2012.03.003

Davidoff, A. M. (2012). Neuroblastoma. Seminars in Pediatric Surgery, 21(1), 2-14. https://doi.org/10.1053/j.sempedsurg.2011.10.009

Dawson, M. A., \& Kouzarides, T. (2012, July 6). Cancer epigenetics: From mechanism to therapy. Cell, Vol. 150, pp. 12-27. https://doi.org/10.1016/j.cell.2012.06.013

Decaesteker, B., Denecker, G., Van Neste, C., Dolman, E. M., Van Loocke, W., Gartlgruber, M., ... Speleman, F. (2018). TBX2 is a neuroblastoma core regulatory circuitry component enhancing MYCN/FOXM1 reactivation of DREAM targets. Nature Communications, 9(1). https://doi.org/10.1038/s41467-018-06699-9

Deneberg, S., Cherif, H., Lazarevic, V., Andersson, P. O., von Euler, M., Juliusson, G., \& Lehmann, S. (2016). An open-label phase I dose-finding study of APR-246 in hematological malignancies. Blood Cancer Journal, 6(7), e447. https://doi.org/10.1038/bcj.2016.60

Dongre, A., \& Weinberg, R. A. (2019, February 1). New insights into the mechanisms of epithelial-mesenchymal transition and implications for cancer. Nature Reviews Molecular Cell Biology, Vol. 20, pp. 69-84. https://doi.org/10.1038/s41580-0180080-4

Dupin, E., Creuzet, S., \& Le Douarin, N. M. (n.d.). The Contribution of the Neural Crest to the Vertebrate Body. Retrieved from https://www.ncbi.nlm.nih.gov/books/NBK6098/

Durbin, A. D., Zimmerman, M. W., Dharia, N. V., Abraham, B. J., Iniguez, A. B., Weichert-Leahey, N., ... Stegmaier, K. (2018, September 1). Selective gene dependencies in MYCN-amplified neuroblastoma include the core transcriptional regulatory circuitry. Nature Genetics, Vol. 50, pp. 1240-1246. https://doi.org/10.1038/s41588-018-0191-z

Eroglu, E., Burkard, T. R., Jiang, Y., Saini, N., Homem, C. C. F., Reichert, H., \& Knoblich, J. A. (2014). SWI/SNF complex prevents lineage reversion and induces temporal patterning in neural stem cells. Cell, 156(6), 1259-1273. https://doi.org/10.1016/j.cell.2014.01.053

Etchevers, H. C., Dupin, E., \& Le Douarin, N. M. (2019). The diverse neural crest: From embryology to human pathology. Development (Cambridge), 146(5). https://doi.org/10.1242/dev.169821

Filbin, M., \& Monje, M. (n.d.). Developmental origins and emerging therapeutic opportunities for childhood cancer. https://doi.org/10.1038/s41591-019-0383-9

Flavahan, W. A., Gaskell, E., \& Bernstein, B. E. (2017, July 21). Epigenetic plasticity and the hallmarks of cancer. Science, Vol. 357. https://doi.org/10.1126/science.aal2380 
Fong, C., Look, A. T., Vogelstein, B., \& Cavenee, W. K. (1992). Loss of Heterozygosity for Chromosomes 1 or 14 Defines Subsets of Advanced Neuroblastomas. Cancer Research, 52(7), 1780-1785. Retrieved from https://cancerres.aacrjournals.org/content/52/7/1780.long

Fong, C. T., Dracopoli, N. C., White, P. S., Merrill, P. T., Griffith, R. C., Housman, D. E., \& Brodeur, G. M. (1989). Loss of heterozygosity for the short arm of chromosome 1 in human neuroblastomas: correlation with N-myc amplification. Proceedings of the National Academy of Sciences of the United States of America, 86(10), 3753-3757. https://doi.org/10.1073/pnas.86.10.3753

Fujita, T., Igarashi, J., Okawa, E. R., Gotoh, T., Manne, J., Kolla, V., ... Brodeur, G. M. (2008). CHD5, a tumor suppressor gene deleted from 1p36.31 in neuroblastomas.

Journal of the National Cancer Institute, 100(13), 940-949. https://doi.org/10.1093/jnci/djn176

Fukuta, M., Nakai, Y., Kirino, K., Nakagawa, M., Sekiguchi, K., Nagata, S., ... Toguchida, J. (2014). Derivation of mesenchymal stromal cells from pluripotent stem cells through a neural crest lineage using small molecule compounds with defined media. PLoS ONE, 9(12), 1-25. https://doi.org/10.1371/journal.pone.0112291

Furlan, A., Dyachuk, V., Kastriti, M. E., Calvo-Enrique, L., Abdo, H., Hadjab, S., ... Adameyko, I. (2017). Multipotent peripheral glial cells generate neuroendocrine cells of the adrenal medulla. Science. https://doi.org/10.1126/science.aal3753

Gao, X., Tate, P., Hu, P., Tjian, R., Skarnes, W. C., \& Wang, Z. (2008). ES cell pluripotency and germ-layer formation require the SWI/SNF chromatin remodeling component BAF250a. Proceedings of the National Academy of Sciences, 105(18), 6656-6661. https://doi.org/10.1073/pnas.0801802105

García-López, J., Wallace, K., Otero, J. H., Olsen, R., Wang, Y. dong, Finkelstein, D., ... Freeman, K. W. (2020). Large 1p36 Deletions Affecting Arid1a Locus Facilitate Mycn-Driven Oncogenesis in Neuroblastoma. Cell Reports, 30(2), 454-464.e5. https://doi.org/10.1016/j.celrep.2019.12.048

Giulino-Roth, L., Wang, K., MacDonald, T. Y., Mathew, S., Tam, Y., Cronin, M. T., ... Cesarman, E. (2012). Targeted genomic sequencing of pediatric Burkitt lymphoma identifies recurrent alterations in antiapoptotic and chromatin-remodeling genes. Blood, 120(26), 5181-5184. https://doi.org/10.1182/blood-2012-06-437624

Gorrini, C., \& Mak, T. W. (2019). Glutathione Metabolism: An Achilles' Heel of ARID1A-Deficient Tumors. Cancer Cell. https://doi.org/10.1016/j.ccell.2019.01.017

Goto, H., Kasahara, K., \& Inagaki, M. (2014). Novel Insights into Chk1 Regulation by Phosphorylation. Cell Structure and Function, 40(1), 43-50. https://doi.org/10.1247/csf.14017

Gröbner, S. N., Worst, B. C., Weischenfeldt, J., Buchhalter, I., Kleinheinz, K., Rudneva, V. A., ... Pfister, S. M. (2018). The landscape of genomic alterations across childhood cancers. Nature. https://doi.org/10.1038/nature25480

Guan, B., Wang, T. L., \& Shih, I. M. (2011). ARID1A, a factor that promotes formation of SWI/SNF-mediated chromatin remodeling, is a tumor suppressor in gynecologic cancers. Cancer Research, 71(21), 6718-6727. https://doi.org/10.1158/00085472.CAN-11-1562 
Ham, J., Costa, C., Sano, R., Lochmann, T. L., Sennott, E. M., Patel, N. U., ... Faber, A. C. (2016). Exploitation of the Apoptosis-Primed State of MYCN-Amplified Neuroblastoma to Develop a Potent and Specific Targeted Therapy Combination. Cancer Cell, 29(2), 159-172. https://doi.org/10.1016/j.ccell.2016.01.002

Han, L., Madan, V., Mayakonda, A., Dakle, P., Woon, T. W., Shyamsunder, P., ... Koeffler, H. P. (2019). Chromatin remodeling mediated by ARID1A is indispensable for normal hematopoiesis in mice. Leukemia. https://doi.org/10.1038/s41375-019-0438-4

Hanahan, D, \& Weinberg, R. A. (2000). The hallmarks of cancer. Cell, 100(1), 57-70. https://doi.org/10.1007/s00262-010-0968-0

Hanahan, Douglas, \& Weinberg, R. A. (2011). Hallmarks of cancer: The next generation. Cell, 144(5), 646-674. https://doi.org/10.1016/j.cell.2011.02.013

Harenza, J. L., Diamond, M. A., Adams, R. N., Song, M. M., Davidson, H. L., Hart, L. S., ... Maris, J. M. (2017). Transcriptomic profiling of 39 commonly-used neuroblastoma cell lines. Scientific Data, 4(1), 170033. https://doi.org/10.1038/sdata.2017.33

Helming, K. C., Wang, X., Wilson, B. G., Vazquez, F., Haswell, J. R., Manchester, H. E., ... Roberts, C. W. M. (2014). ARID1B is a specific vulnerability in ARID1Amutant cancers. Nature Medicine, 20(3), 251-254. https://doi.org/10.1038/nm.3480

Hnisz, D., Abraham, B. J., Lee, T. I., Lau, A., Saint-André, V., Sigova, A. A., .. Young, R. A. (2013). Super-enhancers in the control of cell identity and disease. Cell, 155(4), 934. https://doi.org/10.1016/j.cell.2013.09.053

Hu, H. M., Zhao, X., Kaushik, S., Robillard, L., Barthelet, A., Lin, K. K., ... Bandyopadhyay, S. (2018). A Quantitative Chemotherapy Genetic Interaction Map Reveals Factors Associated with PARP Inhibitor Resistance. Cell Reports, 23(3), 918-929. https://doi.org/10.1016/j.celrep.2018.03.093

Huang, M., Miller, M. L., McHenry, L. K., Zheng, T., Zhen, Q., Ilkhanizadeh, S., ... Weiss, W. A. (2016). Generating trunk neural crest from human pluripotent stem cells. Scientific Reports, 6. https://doi.org/10.1038/srep19727

Huber, K. (2015). Segregation of neuronal and neuroendocrine differentiation in the sympathoadrenal lineage. Cell and Tissue Research, 359(1), 333-341. https://doi.org/10.1007/s00441-014-1947-0

Huether, R., Dong, L., Chen, X., Wu, G., Parker, M., Wei, L., ... Downing, J. R. (2014). The landscape of somatic mutations in epigenetic regulators across 1,000 paediatric cancer genomes. Nature Communications, 5. https://doi.org/10.1038/ncomms4630

Janoueix-Lerosey, I., Novikov, E., Monteiro, M., Gruel, N., Schleiermacher, G., Loriod, B., ... Delattre, O. (2004). Gene expression profiling of 1p35-36 genes in neuroblastoma. Oncogene, 23(35), 5912-5922. https://doi.org/10.1038/sj.onc.1207784

Jiang, M., Stanke, J., \& Lahti, J. M. (2013). The Connections Between Neural Crest Development and Neuroblastoma. In Frontiers in Oncology (Vol. 9). https://doi.org/10.1016/B978-0-12-380916-2.00004-8

Jones, D. T. W., Banito, A., Grünewald, T. G. P., Haber, M., Jäger, N., Kool, M., ... Pfister, S. M. (2019, August 1). Molecular characteristics and therapeutic vulnerabilities across paediatric solid tumours. Nature Reviews Cancer, Vol. 19, pp. 420-438. https://doi.org/10.1038/s41568-019-0169-x 
Jones, S., Li, M., Williams Parsons, D., Zhang, X., Wesseling, J., Kristel, P., ...

Papadopoulos, N. (2012). Somatic mutations in the chromatin remodeling gene

ARID1A occur in several tumor types. Human Mutation, 33(1), 100-103. https://doi.org/10.1002/humu.21633

Jubierre, L., Jiménez, C., Rovira, E., Soriano, A., Sábado, C., Gros, L., ... Segura, M. F. (2018, April 1). Targeting of epigenetic regulators in neuroblastoma. Experimental and Molecular Medicine, Vol. 50. https://doi.org/10.1038/s12276-018-0077-2

Kadoch, C., Hargreaves, D. C., Hodges, C., Elias, L., Ho, L., Ranish, J., \& Crabtree, G. R. (2013). Proteomic and bioinformatic analysis of mammalian SWI/SNF complexes identifies extensive roles in human malignancy. Nature Genetics, 45(6), 592-601. https://doi.org/10.1038/ng.2628

Kelwick, R., Desanlis, I., Wheeler, G. N., \& Edwards, D. R. (2015). The ADAMTS (A Disintegrin and Metalloproteinase with Thrombospondin motifs) family. Genome Biology, 16(1). https://doi.org/10.1186/s13059-015-0676-3

Kim, I., Lee, J. W., Lee, M., Kim, H. S., Chung, H. H., Kim, J. W., ... Seo, J. S. (2018). Genomic landscape of ovarian clear cell carcinoma via whole exome sequencing. Gynecologic Oncology, 148(2), 375-382. https://doi.org/10.1016/j.ygyno.2017.12.005

Knudson, A. G. (1971). Mutation and Cancer: Statistical Study of Retinoblastoma. Proc Natl Acad Sci U S A, 68(4), 820-823. https://doi.org/10.1073/pnas.68.4.820

Kuo, B. R., \& Erickson, C. A. (2010). Regional differences in neural crest morphogenesis. Cell Adhesion \& Migration, 4(4), 567-585. https://doi.org/10.4161/cam.4.4.12890

Lawlor, E. R., \& Thiele, C. J. (2012, May 15). Epigenetic changes in pediatric solid tumors: Promising new targets. Clinical Cancer Research, Vol. 18, pp. 2768-2779. https://doi.org/10.1158/1078-0432.CCR-11-1921

Lei, I., West, J., Yan, Z., Gao, X., Fang, P., Dennis, J. H., ... Wang, Z. (2015). BAF250a protein regulates nucleosome occupancy and histone modifications in priming embryonic stem cell differentiation. Journal of Biological Chemistry, 290(31), 19343-19352. https://doi.org/10.1074/jbc.M115.637389

Lin, C. Y., Lovén, J., Rahl, P. B., Paranal, R. M., Burge, C. B., Bradner, J. E., ... Young, R. A. (2012). Transcriptional amplification in tumor cells with elevated c-Myc. Cell, 151(1), 56-67. https://doi.org/10.1016/j.cell.2012.08.026

Loeb, L. A. (2001). A Mutator Phenotype in Cancer. Cancer Research, 61, 3230-3239.

Lord, C. J., Campbell, J., Ashworth, A., Francis, J., Jones, S. E., Rafiq, R., ... Pemberton, H. N. (2016). ATR inhibitors as a synthetic lethal therapy for tumours deficient in ARID1A. Nature Communications. https://doi.org/10.1038/ncomms 13837

Love, C., Sun, Z., Jima, D., Li, G., Zhang, J., Miles, R., ... Dave, S. S. (2012). The genetic landscape of mutations in Burkitt lymphoma. Nature Genetics, 44(12), 1321-1325. https://doi.org/10.1038/ng.2468

Love, M. I., Huber, W., \& Anders, S. (2014). Moderated estimation of fold change and dispersion for RNA-seq data with DESeq2. Genome Biology, 15(12), 1-21. https://doi.org/10.1186/s13059-014-0550-8

Lu, C., \& Allis, C. D. (2017). SWI/SNF complex in cancer. Nature Genetics. https://doi.org/10.1038/ng.3779 
Ma, X., Liu, Y., Liu, Y., Alexandrov, L. B., Edmonson, M. N., Gawad, C., ... Zhang, J. (2018). Pan-cancer genome and transcriptome analyses of 1,699 paediatric leukaemias and solid tumours. Nature. https://doi.org/10.1038/nature25795

Malynn, B. A., Moreno De Alboran, I., Ná, R., O’hagan, C., Bronson, R., Davidson, L., ... Alt, F. W. (2000). N-myc can functionally replace c-myc in murine development, cellular growth, and differentiation. Genes and Development, 14(11), 1390-1399. Retrieved from https://www.ncbi.nlm.nih.gov/pmc/articles/PMC316670/

Maris, J. M. (2010, June 10). Recent advances in neuroblastoma. New England Journal of Medicine, Vol. 362, p. 2202. https://doi.org/10.1056/nejmra0804577

Maris, J. M., Hogarty, M. D., Bagatell, R., Cohn, S. L., \& Susan. (2007). Neuroblastoma. Lancet, 369, 2016-2020. https://doi.org/10.1016/S0140-6736(07)60983-0

Maris, J. M., \& Matthay, K. K. (1999). Molecular Biology of Neuroblastoma. J Clin Oncol, 17, 2264-2279.

Maris, J. M., White, P. S., Beltinger, C. P., Sulman, E. P., Castleberry, R. P., Shuster, J. J., ... Brodeur, G. M. (1995). Significance of Chromosome 1p Loss of Heterozygosity in Neuroblastoma. Cancer Research, 55(20), 4664-4669. Retrieved from https://cancerres.aacrjournals.org/content/55/20/4664.long

Mathur, R. (2018). ARID1A loss in cancer: Towards a mechanistic understanding. Pharmacology and Therapeutics. https://doi.org/10.1016/j.pharmthera.2018.05.001

Mathur, R., Alver, B. H., San Roman, A. K., Wilson, B. G., Wang, X., Agoston, A. T., ... Roberts, C. W. M. (2017). ARID1A loss impairs enhancer-mediated gene regulation and drives colon cancer in mice. Nature Genetics, 49(2), 296-302. https://doi.org/10.1038/ng.3744

Meacham, C. E., \& Morrison, S. J. (2013). Tumor heterogeneity and cancer cell plasticity. Nature, 501(7467), 328-337. https://doi.org/10.1038/nature12624

Merindol, N., Riquet, A., Szablewski, V., Eliaou, J. F., Puisieux, A., \& Bonnefoy, N. (2014). The emerging role of Twist proteins in hematopoietic cells and hematological malignancies. Blood Cancer Journal, Vol. 4. https://doi.org/10.1038/bcj.2014.22

Mirsky, R., Woodhoo, A., Parkinson, D. B., Arthur-Farraj, P., Bhaskaran, A., \& Jessen, K. R. (2008). Novel signals controlling embryonic Schwann cell development, myelination and dedifferentiation. Journal of the Peripheral Nervous System. https://doi.org/10.1111/j.1529-8027.2008.00168.x

Muzny, D. M., Bainbridge, M. N., Chang, K., Dinh, H. H., Drummond, J. A., Fowler, G., ... Thomson., E. (2012). Comprehensive molecular characterization of human colon and rectal cancer. Nature, 487(7407), 330-337. https://doi.org/10.1038/nature11252

Nagl, N. G., Patsialou, A., Haines, D. S., Dallas, P. B., Beck, G. R., \& Moran, E. (2005). The p270 (ARID1A/SMARCF1) subunit of mammalian SWI/SNF-related complexes is essential for normal cell cycle arrest. Cancer Research, 65(20), 92369244. https://doi.org/10.1158/0008-5472.CAN-05-1225

Nagl, N. G., Zweitzig, D. R., Thimmapaya, B., Beck, G. R., \& Moran, E. (2006). The cmyc gene is a direct target of mammalian SWI/SNF-related complexes during differentiation-associated cell cycle arrest. Cancer Research, 66(3), 1289-1293. https://doi.org/10.1158/0008-5472.CAN-05-3427 
Nakagawara, A., Li, Y., Izumi, H., Muramori, K., Inada, H., \& Nishi, M. (2018, March 1). Neuroblastoma. Japanese Journal of Clinical Oncology, Vol. 48, pp. 214-241. https://doi.org/10.1093/jjco/hyx176

Ogiwara, H., Takahashi, K., Sasaki, M., Kuroda, T., Yoshida, H., Watanabe, R., ... Kohno, T. (2019). Targeting the Vulnerability of Glutathione Metabolism in ARID1A-Deficient Cancers. Cancer Cell. https://doi.org/10.1016/j.ccell.2018.12.009

Okawa, E. R., Gotoh, T., Manne, J., Igarashi, J., Fujita, T., Silverman, K. A., ... Brodeur, G. M. (2008). Expression and sequence analysis of candidates for the 1p36.31 tumor suppressor gene deleted in neuroblastomas. Oncogene, 27(6), 803-810. https://doi.org/10.1038/sj.onc.1210675

Olsen, R. R., Otero, J. H., García-López, J., Wallace, K., Finkelstein, D., Rehg, J. E., ... Freeman, K. W. (2017). MYCN induces neuroblastoma in primary neural crest cells. Oncogene, 36(35), 5075-5082. https://doi.org/10.1038/onc.2017.128

Olsson, A. H., Yang, B. T., Hall, E., Taneera, J., Salehi, A., Nitert, M. D., \& Ling, C. (2011). Decreased expression of genes involved in oxidative phosphorylation in human pancreatic islets from patients with type 2 diabetes. European Journal of Endocrinology, 165(4), 589-595. https://doi.org/10.1530/EJE-11-0282

Park, D. S., Robertson-Tessi, M., Luddy, K. A., Maini, P. K., Bonsall, M. B., Gatenby, R. A., \& Anderson, A. R. A. (2019). The goldilocks window of personalized chemotherapy: Getting the immune response just right. Cancer Research, 79(20), 5302-5315. https://doi.org/10.1158/0008-5472.CAN-18-3712

Park, J. R., Bagatell, R., London, W. B., Maris, J. M., Cohn, S. L., Mattay, K. M., \& Hogarty, M. (2013, June). Children's Oncology Group's 2013 blueprint for research: Neuroblastoma. Pediatric Blood and Cancer, Vol. 60, pp. 985-993. https://doi.org/10.1002/pbc.24433

Park, J. R., Eggert, A., \& Caron, H. (2010). Neuroblastoma: Biology, Prognosis, and Treatment. Hematology/Oncology Clinics of North America. https://doi.org/10.1016/j.hoc.2009.11.011

Parmigiani, G., Garrett-Mayer, E. S., Anbazhagan, R., \& Gabrielson, E. (2004). A CrossStudy Comparison of Gene Expression Studies for the Molecular Classification of Lung Cancer. Clinical Cancer Research, 10(9), 2922-2927. https://doi.org/10.1158/1078-0432.CCR-03-0490

Peuchmaur, M., D’Amore, E. S. G., Joshi, V. V., Hata, J. I., Roald, B., Dehner, L. P., ... Shimada, H. (2003, November 15). Revision of the International Neuroblastoma Pathology Classification: Confirmation of Favorable and Unfavorable Prognostic Subsets in Ganglioneuroblastoma, Nodular. Cancer, Vol. 98, pp. 2274-2281. https://doi.org/10.1002/cncr.11773

Pinto, N. R., Applebaum, M. A., Volchenboum, S. L., Matthay, K. K., London, W. B., Ambros, P. F., ... Cohn, S. L. (2015, September 20). Advances in risk classification and treatment strategies for neuroblastoma. Journal of Clinical Oncology, Vol. 33, pp. 3008-3017. https://doi.org/10.1200/JCO.2014.59.4648

Pugh, T. J., Morozova, O., Attiyeh, E. F., Asgharzadeh, S., Wei, J. S., Auclair, D., ... Maris, J. M. (2013). The genetic landscape of high-risk neuroblastoma. Nature Genetics, 45(3), 279-284. https://doi.org/10.1038/ng.2529 
Quintana, E., Shackleton, M., Sabel, M. S., Fullen, D. R., Johnson, T. M., \& Morrison, S. J. (2008). Efficient tumour formation by single human melanoma cells. Nature, 456(7222), 593-598. https://doi.org/10.1038/nature07567

R Core Team. (2019). R: A language and environment for statistical computing. Retrieved from https://www.r-project.org/

Raab, J. R., Resnick, S., \& Magnuson, T. (2015). Genome-Wide Transcriptional Regulation Mediated by Biochemically Distinct SWI/SNF Complexes. PLoS Genetics. https://doi.org/10.1371/journal.pgen.1005748

Rickman, D. S., Schulte, J. H., \& Eilers, M. (2018, February 1). The expanding world of N-MYC-driven tumors. Cancer Discovery, Vol. 8, pp. 150-164. https://doi.org/10.1158/2159-8290.CD-17-0273

Ruiz-Pérez, M. V., Henley, A. B., \& Arsenian-Henriksson, M. (2017, April 1). The MYCN protein in health and disease. Genes, Vol. 8. https://doi.org/10.3390/genes8040113

Sausen, M., Leary, R. J., Jones, S., Wu, J., Reynolds, C. P., Liu, X., ... Hogarty, M. D. (2013). Integrated genomic analyses identify ARID1A and ARID1B alterations in the childhood cancer neuroblastoma. Nature Genetics, 45(1), 12-17. https://doi.org/10.1038/ng.2493

Schulte, J., \& Eggert, A. (2015). Neuroblastoma. Critical Reviews in Oncogenesis, 20(34), 245-270.

Schwab, M., Praml, C., \& Amler, L. C. (1996). Genomic instability in 1p and human malignancies. Genes Chromosomes and Cancer, 16(4), 211-229. https://doi.org/10.1002/(SICI)1098-2264(199608)16:4<211::AID-GCC1>3.0.CO;20

Selmi, A., de Saint-Jean, M., Jallas, A. C., Garin, E., Hogarty, M. D., Bénard, J., ... Valsesia-Wittmann, S. (2015). TWIST1 is a direct transcriptional target of MYCN and MYC in neuroblastoma. Cancer Letters. https://doi.org/10.1016/j.canlet.2014.11.056

Shen, J., Ju, Z., Zhao, W., Wang, L., Peng, Y., Ge, Z., ... Peng, G. (2018). ARID1A deficiency promotes mutability and potentiates therapeutic antitumor immunity unleashed by immune checkpoint blockade. Nature Medicine. https://doi.org/10.1038/s41591-018-0012-Z

Shen, J., Peng, Y., Wei, L., Zhang, W., Yang, L., Lan, L., ... Peng, G. (2015). ARID1A Deficiency Impairs the DNA Damage Checkpoint and Sensitizes Cells to PARP Inhibitors. Cancer Discovery. https://doi.org/10.1158/2159-8290.CD-14-0849

Shimada, H., Ambros, I. M., Dehner, L. P., Hata, J.-I., Joshi, V. V, Roald, B., ... Castleberry, R. P. (1999). The International Neuroblastoma Pathology Classification (the Shimada System). American Cancer Society, 86(2), 364-372. Retrieved from https://www.ncbi.nlm.nih.gov/pubmed/10421273

Soldatov, R., Kaucka, M., Kastriti, M. E., Petersen, J., Chontorotzea, T., Englmaier, L., ... Adameyko, I. (2019). Spatiotemporal structure of cell fate decisions in murine neural crest. Science, 364(6444). https://doi.org/10.1126/science.aas9536

Son, E. Y., \& Crabtree, G. R. (2014). The role of BAF (mSWI/SNF) complexes in mammalian neural development. American Journal of Medical Genetics, Part C: Seminars in Medical Genetics, 166(3), 333-349. https://doi.org/10.1002/ajmg.c.31416 
Squire, L., Berg, D., Bloom, F. E., du Lac, S., Ghosh, A., Squire, L. R., ... Zigmond, M. (Eds.). (2003). Neurogenesis and Migration. In Fundamental Neuroscience (Second Edition, pp. 391-416). Elsevier Science.

Subramanian, A., Tamayo, P., Mootha, V. K., Mukherjee, S., Ebert, B. L., Gillette, M. A., ... Mesirov, J. P. (2005). Gene set enrichment analysis: A knowledge-based approach for interpreting genome-wide expression profiles. Proceedings of the National Academy of Sciences, 102(43), 15545-15550. https://doi.org/10.1073

Sun, X., Chuang, J., Kanchwala, M., Wu, L., Celen, C., Liang, H., ... Xu, J. (2017). Suppression of the SWI/SNF Component Arid1a Promotes Mammalian Regeneration. Cell Stem Cell, 18(4), 456-466. https://doi.org/10.1016/j.stem.2016.03.001.Suppression

Sun, X., Wang, S. C., Wei, Y., Luo, X., Jia, Y., Li, L., ... Zhu, H. (2017). Arid1a Has Context-Dependent Oncogenic and Tumor Suppressor Functions in Liver Cancer. Cancer Cell, 32(5), 574-589.e6. https://doi.org/10.1016/j.ccell.2017.10.007

Tolbert, V. P., \& Matthay, K. K. (2018, May 1). Neuroblastoma: clinical and biological approach to risk stratification and treatment. Cell and Tissue Research, Vol. 372, pp. 195-209. https://doi.org/10.1007/s00441-018-2821-2

Tomlinson, I. P. M., Lambros, M. B. K., Roylance, R. R., \& Cleton-Jansen, A. M. (2002). Loss of heterozygosity analysis: Practically and conceptually flawed? Genes Chromosomes and Cancer, 34(4), 349-353. https://doi.org/10.1002/gcc.10085

Tsubota, S., \& Kadomatsu, K. (2018, May 1). Origin and initiation mechanisms of neuroblastoma. Cell and Tissue Research, Vol. 372, pp. 211-221. https://doi.org/10.1007/s00441-018-2796-z

Valsesia-Wittmann, S., Magdeleine, M., Dupasquier, S., Garin, E., Jallas, A. C., Combaret, V., ... Puisieux, A. (2004). Oncogenic cooperation between H-Twist and N-Myc overrides failsafe programs in cancer cells. Cancer Cell, 6(6), 625-630. https://doi.org/10.1016/j.ccr.2004.09.033

Van Dang, C., Lorenzin, F., Benary, U., Baluapuri, A., Walz, S., Anna Jung, L., ... Wolf, E. (2016). Different promoter affinities account for specificity in MYC-dependent gene regulation. https://doi.org/10.7554/eLife.15161.001

van Groningen, T., Kroster, J., Valentijn, L. J., Zwijnenburg, D. A., Akogul, N., Hasselt, N. E., ... Versteeg, R. (2017). Neuroblastoma is composed of two super-enhancerassociated differentiation states. Nature Genetics. https://doi.org/10.1038/ng.3899

Versteeg, R., Caron, H., Cheng, N. C., van der Drift, P., Slater, R., Westerveld, A., ... Speleman, F. (1995). 1p36: Every Subband a Suppressor? European Journal of Cancer, 31(4), 538-541. https://doi.org/10.1016/0959-8049(95)00037-j

Vincentz, J. W., Firulli, B. A., Lin, A., Spicer, D. B., Howard, M. J., \& Firulli, A. B. (2013). Twist1 Controls a Cell-Specification Switch Governing Cell Fate Decisions within the Cardiac Neural Crest. PLoS Genetics, 9(3).

https://doi.org/10.1371/journal.pgen.1003405

Vogelstein, B., Papadopoulos, N., \& Velculescu, V. (2013). Cancer Genome Landscapes. Science, 339(6127), 1546-1558. https://doi.org/10.1126/science.1235122

Wang, L., Tan, T. K., Durbin, A. D., Zimmerman, M. W., Abraham, B. J., Tan, S. H., ... Sanda, T. (2019). ASCL1 is a MYCN- and LMO1-dependent member of the adrenergic neuroblastoma core regulatory circuitry. Nature Communications, 10(1). https://doi.org/10.1038/s41467-019-13515-5 
Wang, S. C., Nassour, I., Xiao, S., Zhang, S., Luo, X., Lee, J., ... Zhu, H. (2019). SWI/SNF component ARID1A restrains pancreatic neoplasia formation. Gut, 68(7), 1259-1270. https://doi.org/10.1136/gutjnl-2017-315490

Wang, W., Friedland, S. C., Guo, B., O’Dell, M. R., Alexander, W. B., Whitney-Miller, C. L., ... Hezel, A. F. (2019). ARID1A, a SWI/SNF subunit, is critical to acinar cell homeostasis and regeneration and is a barrier to transformation and epithelialmesenchymal transition in the pancreas. Gut, 68(7), 1245-1258. https://doi.org/10.1136/gutjnl-2017-315541

Weiss, W. A., Aldape, K., Mohapatra, G., Feuerstein, B. G., \& Michael Bishop, J. (1997). Targeted expression of MYCN causes neuroblastoma in transgenic mice. The EMBO Journal, 16(11), 2985-2995. https://doi.org/10.1093/emboj/16.11.2985

Weissman, B., \& Knudsen, K. E. (2009, November 1). Hijacking the chromatin remodeling machinery: Impact of SWI/SNF perturbations in cancer. Cancer Research, Vol. 69, pp. 8223-8230. https://doi.org/10.1158/0008-5472.CAN-092166

White, P. S., Thompson, P. M., Gotoh, T., Okawa, E. R., Igarashi, J., Kok, M., ... Brodeur, G. M. (2005). Definition and characterization of a region of $1 \mathrm{p} 36.3$ consistently deleted in neuroblastoma. Oncogene, 24(16), 2684-2694. https://doi.org/10.1038/sj.onc.1208306

Whyte, W. A., Orlando, D. A., Hnisz, D., Abraham, B. J., Lin, C. Y., Kagey, M. H., ... Young, R. A. (2013). Master transcription factors and mediator establish superenhancers at key cell identity genes. Cell, 153(2), 307-319. https://doi.org/10.1016/j.cell.2013.03.035

Wiegand, K. C., Shah, S. P., Al-Agha, O. M., Zhao, Y., Tse, K., Zeng, T., ... Huntsman, D. G. (2010). ARID1A mutations in endometriosis-associated ovarian carcinomas. New England Journal of Medicine, 363(16), 1532-1543. https://doi.org/10.1056/NEJMoa1008433

Wilkerson, M. D., \& Hayes, D. N. (2010). ConsensusClusterPlus: A class discovery tool with confidence assessments and item tracking. Bioinformatics, 26(12), 1572-1573. https://doi.org/10.1093/bioinformatics/btq170

Wilson, B. G., Wang, X., Shen, X., McKenna, E. S., Lemieux, M. E., Cho, Y. J., ... Roberts, C. W. M. (2010). Epigenetic antagonism between polycomb and SWI/SNF complexes during oncogenic transformation. Cancer Cell. https://doi.org/10.1016/j.ccr.2010.09.006

Wilson, M. R., Reske, J. J., Holladay, J., Wilber, G. E., Rhodes, M., Koeman, J., ... Chandler, R. L. (2019). ARID1A and PI3-kinase pathway mutations in the endometrium drive epithelial transdifferentiation and collective invasion. Nature Communications, 10(1), 3554. https://doi.org/10.1038/s41467-019-11403-6

Witkiewicz, A. K., McMillan, E. A., Balaji, U., Baek, G. H., Lin, W. C., Mansour, J., ... Knudsen, E. S. (2015). Whole-exome sequencing of pancreatic cancer defines genetic diversity and therapeutic targets. Nature Communications, 6 . https://doi.org/10.1038/ncomms 7744

Wong, D. J., Liu, H., Ridky, T. W., Cassarino, D., Segal, E., \& Chang, H. Y. (2008). Module Map of Stem Cell Genes Guides Creation of Epithelial Cancer Stem Cells. Cell Stem Cell, 2(4), 333-344. https://doi.org/10.1016/j.stem.2008.02.009 
Wong, D. J., Liu, H., Ridky, T. W., Cassarino, D., Segal, E., \& Chang, Y. (2009). Module map of stem cell genes guides creation of epithelial cancer stem cells. 2(4), 333344. https://doi.org/10.1016/j.stem.2008.02.009

Wu, J. N., \& Roberts, C. W. M. (2013). ARID1A mutations in cancer: Another epigenetic tumor suppressor? Cancer Discovery, 3(1), 35-43. https://doi.org/10.1158/2159-8290.CD-12-0361

Wu, R. C., Wang, T. L., \& Shih, I. M. (2014). The emerging roles of ARID1A in tumor suppression. Cancer Biology and Therapy, 15(6), 655-664. https://doi.org/10.4161/cbt.28411

Yang, J., Mani, S. A., Liu Donaher, J., Ramaswamy, S., Itzykson, R. A., Come, C., ... Weinberg, R. A. (2004). Twist, a Master Regulator of Morphogenesis, Plays an Essential Role in Tumor Metastasis. 117(7), 927-939. https://doi.org/10.1016/j.cell.2004.06.006

Yoshida, B. A., Sokoloff, M. M., Welch, D. R., \& Rinker-Schaeffer, C. W. (2000). Metastasis-Suppressor Genes: a Review and Perspective on an Emerging Field. Journal of the National Cancer Institute, 92(21), 1717-1730. https://doi.org/10.1093/jnci/92.21.1717

Young, R. A. (2011, March 18). Control of the embryonic stem cell state. Cell, Vol. 144, pp. 940-954. https://doi.org/10.1016/j.cell.2011.01.032

Yu, A. L., Gilman, A. L., Fevzi Ozkaynak, M., London, W. B., Kreissman, S. G., Chen, H. X., ... Sondel, P. M. (2010). Anti-GD2 Antibody with GM-CSF, Interleukin-2, and Isotretinoin for Neuroblastoma. N Engl J Med, 363(14), 1324-1358. https://doi.org/10.1056/NEJMoa0911123

Zeid, R., Lawlor, M. A., Poon, E., Reyes, J. M., Fulciniti, M., Lopez, M. A., ... Bradner, J. E. (2018). Enhancer invasion shapes MYCN-dependent transcriptional amplification in neuroblastoma. Nature Genetics. https://doi.org/10.1038/s41588018-0044-9

Zhu, S., Lee, J. S., Guo, F., Shin, J., Perez-Atayde, A. R., Kutok, J. L., ... Look, A. T. (2012). Activated ALK Collaborates with MYCN in Neuroblastoma Pathogenesis. Cancer Cell, 21(3), 362-373. https://doi.org/10.1016/j.ccr.2012.02.010

Zimmerman, K. A., Yancopoulos, G. D., Collum, R. G., Smith, R. K., Kohl, N. E., Denis, K. A., ... Alt, F. W. (1986). Differential expression of myc family genes during murine development. Nature, 319(6056), 780-783.

https://doi.org/10.1038/319780a0 


\section{APPENDIX A. ADDITIONAL MATERIALS AND METHODS TECHNICAL INFORMATION}

Table A-1. Predicted ARID1A genetic status in common neuroblastoma cell lines.

\begin{tabular}{|c|c|c|c|c|}
\hline \multirow[b]{2}{*}{ Cell Line } & \multicolumn{2}{|c|}{ Sequenced Genetic Event } & \multicolumn{2}{|c|}{ Predicted Protein Status } \\
\hline & MYCN & 1p36 LOH & N-MYC & ARID1A \\
\hline SK-N-AS & Non-Amp & Loss p36.22-36.32 & WT & WT \\
\hline SH-SY5Y & Non-Amp & None & & \\
\hline NBL-S & Non-Amp & None & & \\
\hline SK-N-FI & Non-Amp & None & & \\
\hline SK-N-SH & Non-Amp & None & & \\
\hline NB-16 & Non-Amp & None & & \\
\hline NB-EBc1 & Non-Amp & Loss p35.2-pter & WT & LOH \\
\hline NB-69 & Non-Amp & Loss p13.3-pter & & \\
\hline NB-1,691 & Amp & None & Amp & WT \\
\hline SK-N-DZ & Amp & None & & \\
\hline CHP-134 & Amp & $\begin{array}{l}\text { LOH p32.3-pter; Gain p34.3- } \\
\text { p36.22; Loss p36.22-pter }\end{array}$ & Amp & $\mathrm{LOH}$ \\
\hline IMR-05 & Amp & Gain+LOH p32.3-pter & & \\
\hline IMR-32 & Amp & Loss p32.3-pter & & \\
\hline KELLY & Amp & $\begin{array}{l}\text { LOH p21.3-pter; Loss p36.32; } \\
\text { Gain p36.33 }\end{array}$ & & \\
\hline LA-N-5 & Amp & Loss p33-pter & & \\
\hline NB-1 & Amp & Loss p32.2-pter & & \\
\hline NB-1,643 & Amp & Loss p34.2-pter & & \\
\hline NB-SD & Amp & Loss p21.3-pter & & \\
\hline NGP & Amp & cnLOH p32.3-pter & & \\
\hline NLF & Amp & Loss p32.2-pter & & \\
\hline SK-N-BE(2) & Amp & cnLOH p21.3-pter & & \\
\hline SK-N-BE(2)-C & Amp & cnLOH p21.3-pter & & \\
\hline NB-SD & Amp & Loss p21.3-pter & & \\
\hline NGP & Amp & cnLOH p32.3-pter & & \\
\hline NLF & Amp & Loss p32.2-pter & & \\
\hline NMB & Amp & cnLOH p34.2-pter & & \\
\hline SMS-KAN & Amp & Loss p13.3-pter & & \\
\hline SMS-SAN & Amp & Loss p32.3-pter & & \\
\hline
\end{tabular}

Notes: $\mathrm{WT}=$ wild-type, $\mathrm{Amp}=\mathrm{MYCN}$ gene amplification, $\mathrm{LOH}=$ loss of heterozygosity, pter $=$ p-terminal, $\mathrm{cn}=$ copy number.

Modified with open access permission. License

https://creativecommons.org/licenses/by/4.0/. Harenza, J. L., Diamond, M. A., Adams, R. 


\section{Table A-1. Continued.}

N., Song, M. M., Davidson, H. L., Hart, L. S., ... Maris, J. M. (2017). Transcriptomic profiling of 39 commonly-used neuroblastoma cell lines. Scientific Data, 4(1), 170033. https://doi.org/10.1038/sdata.2017.33 
Table A-2. Immunohistochemistry primary antibodies, type, concentrations, supplier and technical procedures for visualization.

\begin{tabular}{|c|c|c|c|}
\hline Antibody & Type & Conc. & Supplier \\
\hline ARID1A & Rabbit poly & $1: 25$ & Sigma-Aldrich, HPA005456 ${ }^{\mathrm{a}}$ \\
\hline BAF47 (Clone 25) & Mouse $\operatorname{IgG}_{2 \alpha}$ & $1: 1000$ & BD Biosciences, $612111^{\mathrm{b}}$ \\
\hline Cleaved-CASPASE 3 & Rabbit poly & $1: 500$ & BioCare Medical, CP229C ${ }^{b}$ \\
\hline GFAP & Rabbit poly & $1: 4000$ & $\mathrm{DAKO}, \mathrm{Z} 0334^{\mathrm{b}}$ \\
\hline Phospho-Histone H2A.X & Rabbit mono & $1: 200$ & Cell Signaling, $9718^{\mathrm{b}}$ \\
\hline KI67 (Clone SP6) & Rabbit mono & $1: 200$ & ThermoFisher, RM-9106 ${ }^{\mathrm{b}}$ \\
\hline MAP2 & Rabbit poly & $1: 250$ & Millipore, AB5622 ${ }^{\mathrm{c}}$ \\
\hline PHOX2B & Rabbit poly & $1: 100$ & Abcam, ab183741 b \\
\hline Phospho-Histone H3 (S10) & Rabbit poly & $1: 200$ & Bethyl laboratories, IHC-00061 a \\
\hline Synaptophysin & Rabbit poly & $1: 400$ & Spring Biosciences, E2172 b \\
\hline S100 & Rabbit poly & $1: 2000$ & DAKO, Z031129 b \\
\hline Tyrosine Hydroxylase (TH) & Rabbit poly & $1: 500$ & Millipore, AB152 b \\
\hline Tubulin $\beta 3$ (TUJ1) & $\begin{array}{l}\text { Mouse } \\
\operatorname{IgG}_{2 \alpha, \kappa}\end{array}$ & 1:9000 & Biolegend, $801202^{\mathrm{a}}$ \\
\hline
\end{tabular}

\footnotetext{
${ }^{a}$ Heat-induced epitope retrieval, Cell conditioning media 2 (Ventana Medical Systems, Tucson, AZ), 32 minutes; Visualization with DISCOVERY OmniMap anti-Rb HRP (760-4311; Ventana Medical Systems), DISCOVERY ChromoMap DAB kit (760-159; Ventana Medical Systems)

${ }^{\mathrm{b}}$ Heat-induced epitope retrieval, Cell conditioning media 1 (Ventana Medical Systems, Tucson, AZ); Visualization with biotinylated rabbit anti-mouse IgG secondary antibody (Abcam, ab133469), DISCOVERY OmniMap anti-Rb HRP (760-4311; Ventana Medical Systems), DISCOVERY ChromoMap DAB kit (760-159; Ventana Medical Systems) ${ }^{\mathrm{c}}$ Heat-induced epitope retrieval, Epitope Retrieval solution 1 (ER1), 20 minutes; Visualization with rabbit anti-goat (BA-5000; Vector Laboratories), Bond Polymer Refine Detection (DS9800, Leica Biosystems).

Notes: conc $=$ concentration, mono $=$ monoclonal, poly $=$ polyclonal
} 
Table A-3. Immunoblotting antibody information.

\begin{tabular}{lc}
\hline & $\begin{array}{c}\text { Protein } \\
\text { Size (KDa) }\end{array}$ \\
\hline anti-total ATM (D2E2) rabbit mAb CST \#2873 & 350 \\
anti-phos ATM (Ser1981) (D6H9) rabbit mAb CST \#5883 & 350 \\
anti-ATR (phos T1989) (1:500) (ab227851) rabbit pAb & 300 \\
anti-phos ATR (1:500) Ser428 rabbit pAb CST \#2853 & 300 \\
anti-total ATR (1:1000) rabbit mAb E153S CST \#13934; 5\% BSA in PBST & 300 \\
anti-total Chk1 (2G1D5) mouse mAb CST \#2360 (1:2000) & 56 \\
anti-Phos Chk1 (1:500) (Ser317) (D12H3) XP® rabbit mAb CST \#12302 & 56 \\
anti- Chk2 rabbit pAb CST \#2662 & 62 \\
anti-Phos-Chk2 (Thr68) (C13C1) rabbit mAb CST \#2197 (reacts w H) & 62 \\
anti-toal Histone H2A.X rabbit (c term) pAb CST \#2595 (1:2000) & 15 \\
anti-phos-H2A.X (Ser139) (1:2000) mouse mAb, JBW301 Millipore 05-66 & 17 \\
anti-p53 (1C12) Ser20 mouse mAb CST \#2524 & 53 \\
anti-p21WAF1/Cip1 mouse mAb Sigma \#P1484 & 31 \\
anti-Caspase-3 rabbit pAb CST \#9662 & $35,19,17$ \\
anti-Cleaved Caspase-3 (Asp175) (5A1E) rabbit mAb \#9664 & 19,17 \\
anti-ARID1A (1:2000) rabbit mAb CST; 5\% BSA in PBST, CST 12354 & 250 \\
anti-N-MYC (1:1000) rabbit pAb; 5\%BSA in PBST, CST \#9405 & 62 \\
anti-53a (1:2500) rabbit pAb bethyl A301-391A 5\% BSA in PBST & 53 \\
anti-53b (1:2500) rabbit mAb ab180927 & 53 \\
anti-BAF 155 (SMARCC1) (D7F8S) CST 11956 rabbit mAb & 155 \\
anti-actin (1:10:000) ac-15 Sigma mouse mAb; 5\% BSA in PBST & 42 \\
\hline
\end{tabular}

Notes: $\mathrm{mAb}=$ monoclonal antibody, $\mathrm{pAb}=$ polyclonal antibody, phos $=$ phosphorylated 


\section{APPENDIX B. SUPPLEMENTAL EXPERIMENTAL FIGURES}

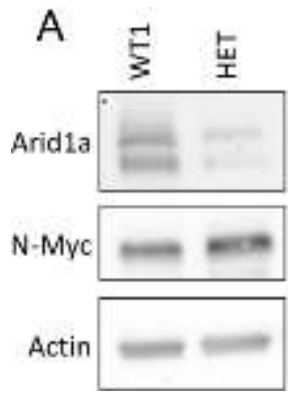

Figure B-1. Comparison of ARID1A and N-MYC protein levels in WT1 and HET primary NCCs.

Western analysis of protein levels prior to NCC injection into mice.

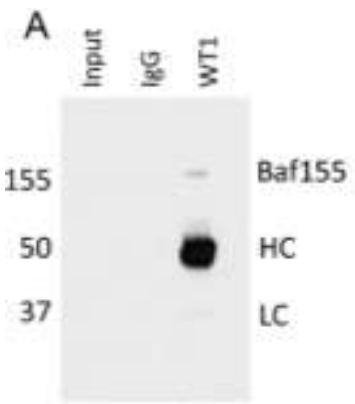

Light Exposure

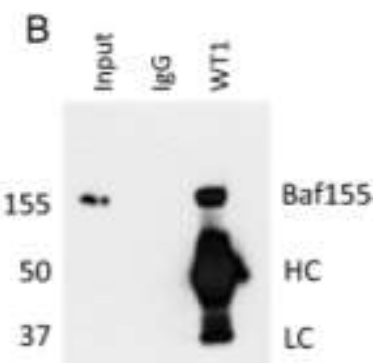

Dark Exposure

Figure B-2 Enrichment of BAF155 protein following immunoprecipitation and immunoblotting.

Immunoprecipitation of SWI/SNF using an anti-BAF155 antibody to pull down the complex using a core subunit of BAF and pBAF complexes. (A) Light exposure. (B) Dark exposure. 


\section{VITA}

Kirby A. Wallace, daughter of Truman R. Wallace and Jill J. Wallace, was born in Danville, Alabama in 1990. Following her graduation from Danville High School in 2009 , she began working as a research intern in the first Biotrain internship program at the Hudson Alpha Institute for Biotechnology at EGEN, Inc before transitioning to a parttime research technician position that she held for five years. In the fall of 2009, she pursued an undergraduate degree at the University of Alabama in Huntsville and majored in biology with a minor in chemistry. She was awarded the Academic Excellence Scholarship, UAH License Tag Scholarship, and Palumbo Endowed Scholarship. In 2014, she matriculated into the Integrated Biomedical Sciences program Cancer and Developmental Biology track in the College of Graduate Health Sciences at The University of Tennessee Health Science Center (UTHSC). She was awarded the Belz Family Scholarship, an Imhotep Society Membership, and she served as president of the Graduate Health Sciences Executive Council and vice president of the Student Government Executive Council at UTHSC. She expects to complete her doctoral degree by May of 2020 . 